\title{
Structure-optimized interpolymer polyphosphazene complexes for effective gene delivery against glioblastoma
}

Authors: Wei-Hsin Hsu, ${ }^{1,2}$ Pilar Sánchez-Gómez, ${ }^{3}$ Esther Gomez-Ibarlucea, ${ }^{1}$ Delyan P. Ivanov, ${ }^{4}$

Ruman Rahman, ${ }^{4}$ Anna M. Grabowska, ${ }^{4}$ Noemi Csaba, ${ }^{1}$ Cameron Alexander ${ }^{2 *}$ and Marcos GarciaFuentes $^{1 *}$

*Corresponding authors: Cameron Alexander (cameron.alexander@nottingham.ac.uk); Marcos Garcia-Fuentes (marcos.garcia@usc.es)

\section{Affiliations}

1 - Center for Research in Molecular Medicine and Chronic Diseases (CIMUS), Universidade de Santiago de Compostela, Spain.

2 - Division of Molecular Therapeutics and Formulation, School of Pharmacy, University of Nottingham, Nottingham NG7 2RD, UK

3 - Neurooncology Unit, Instituto de Salud Carlos III (UFIEC), Madrid, Spain

4 - Division of Cancer and Stem Cells, School of Medicine, Queen's Medical Centre, University of Nottingham, Nottingham NG7 2RD, UK

Keywords: Poly(phosphazene)s, click chemistry, polymeric gene delivery, siRNA delivery, glioblastoma, tumor initiating cells 


\begin{abstract}
Safe and efficient gene delivery vectors would enhance the prospects for polynucleotide-based therapies. Herein we describe a new approach towards structurally-optimized gene vector design based on the preparation of clickable poly(allylamino-phosphazene)s that can be converted to several cationic and anionic derivatives via thiol-ene addition. Simultaneous co-incubation of alkylamineand alkylcarboxylate-poly(phosphazenes) with polynucleotides generated binary-polyelectrolyte nanoparticles. Screening of a series of these complexes for transfection in glioblastoma cells showed that the inclusion of 6-mercaptohexanoic acid substituted poly(phosphazene)s in the complexes resulted in 6-fold and 19-fold higher luciferase expression in U87MG cells and primary GBM1 cellline, respectively. This effect was attributed to the specific ionization properties of this materials that improved polyplex intracellular trafficking. Transfection in 3D-spheroid models and subcutaneous xenograft U87MG tumors confirmed higher transgene expression for the binary cationic/anionic poly(phosphazene) complexes compared to the related polycation-pDNA complexes and to PEIpDNA complexes. The data also indicated a notable capacity of the mixed complexes to deliver genes to the inner cores of tumor spheroids. Extension of this approach to siRNA delivery showed that the mixed poly(phosphazene) complexes were able to silence DYRK1A, a gene implicated in tumor initiation and progression, reducing U87MG cell renewal in vitro and delaying tumor growth in vivo.
\end{abstract}




\section{Introduction}

Therapies based on polynucleotides offer much promise but require more efficient and safer gene carriers ${ }^{[1]}$. While viral-based gene delivery is the most advanced in terms of the number of clinical trials ${ }^{[2]}$, there are still concerns over the long-term safety of viral vectors, and many of the new RNA-based drugs are not easy to integrate in virus-based delivery constructs. Non-viral systems such as polymer-based gene vehicles have provided a number of promising results, but there remain several limitations for their generalized application ${ }^{[3]}$. Design criteria that remain unfulfilled by most synthetic polymer gene carriers include: (a) biocompatibility and tunable biodegradability, (b) selective biodistribution and adequate pharmacokinetics, (c) ability to surmount tissue and extracellular barriers, (d) transport across intracellular barriers and organelle-specific release.

Poly(phosphazene)s are a class of biodegradable materials that could provide these urgently needed characteristics to gene carriers. Pioneering work from the Allcock group established some of the key pharmaceutical parameters for these materials ${ }^{[4]}$, and recent studies have shown that poly(phosphazene)s and their variants can effectively deliver small drug molecules, proteins and nucleic acids in a number of therapeutic settings ${ }^{[5]}$. The versatility of poly(phosphazene) chemistry derives from the possibilities to modify a precursor, poly(dichlorophosphazene), by nucleophilic substitution. However, when preparing polymers for gene delivery, this synthetic route is problematic because most functional candidates display more than two nucleophilic centers, which can result in general crosslinking of the material during derivatization reactions leading to precipitation of the partially substituted polymer. Recently, a few strategies to introduce "click" handles in poly(phosphazene)s have been reported ${ }^{[6]}$, which might be used to circumvent this problem. In this study, we have developed a poly(phosphazene) platform, containing side-chain double-bond repeating units, and prepared a series of cationic and anionic derivatives of these via thiol-ene grafting chemistries using $\alpha, \omega$-aminoalkanethiols and $\alpha, \omega$-carboxylatoalkanethiols. By combining these materials in the presence of nucleic acids, we aimed to generate mixed polyelectrolyte complexes with sufficient positive charge to bind polynucleotides and promote cell internalization, but with the 
capacity to destabilize cell membranes in response to $\mathrm{pH}$, and at the same time to minimize polycation-induced toxicity. These optimized complexes were expected to escape more effectively than conventional polycation/nucleic acid complexes from the endolysosomal compartment, which has been considered to be the most significant intracellular barrier to effective gene delivery ${ }^{[3 b, 7]}$.

Accordingly, we have applied these gene carriers to deliver nucleic acids as a potential means to treat glioblastoma (GBM), which is a highly aggressive and malignant cancer usually classified as grade IV by the World Health Organization (WHO), in view of patients' short median survival time after diagnosis ( $\leq 14$ months) ${ }^{[8]}$. While this prognosis has improved slightly by the introduction of the Stupp protocol treatment, i.e. radiotherapy plus temozolomide (TMZ) after surgical resection of diagnosed tumors ${ }^{[9]}$, the presence of highly-resistant tumor initiating cells (TIC) still results in limited treatment options for most patients, leading to tumor relapse and failure of the therapy. There is an urgent need for better treatment strategies, and delivery of either suicide genes or gene knockdown by siRNA are possible means by which glioblastomas could be effectively targeted. For example, previous studies have shown that inhibition of dual-specificity tyrosine phosphorylation-regulated kinase (DYRK1A) caused a loss of self-renewal capacity in several primary GBM TIC cells through preventing endocytosis-mediated degradation of epidermal growth factor receptor (EGFR) by the phosphorylation-required modulator Sprouty2 ${ }^{[10]}$. In addition, advances in surgical resection for GBM-diagnosed patients are allowing for promising strategies for in situ post-operative administration, for example the introduction of sustained-release wafers to release anti-cancer agents in situ. ${ }^{[11]}$. In this work, we applied the poly(phosphazene) platform to deliver siRNA against DYRK1A to GBM tumors via intratumor administration and report a first proof of therapeutic efficacy in vivo models and anti-neoplastic treatment.

\section{Materials and Methods}

\subsection{Materials}

Hexachlorocyclotriphosphazene (99\%), aluminum chloride (99.99\%), triethylamine (TEA), cysteamine, 2-(dimethylamino)ethanethiol hydrochloride (DMAES), 2,2-dimethoxy-2- 
phenylacetophenone (DMPA), branched poly(ethyleneimine) Mw 25K (PEI), 3-mercaptopropionic acid (3MPA), 4-mercaptobutyric acid (4MBA), 6-mercaptohexanoic acid (6MHA), 8mercaptooctanoic acid (8MOA), heparin sodium salt (from porcine intestinal mucosa), anhydrous tetrahydrofuran (THF), 2,2,2-trifluoroethanol (TFE), anhydrous diethylene glycol dimethyl ether (Diglyme), ethidium bromide (EtBr), Poly-L-lysine (Mw 30K-70K Da) were all purchased from Sigma-Aldrich and used as received.

\subsection{Synthesis of the precursor poly(allylamino-phosphazene)}

Poly(dichlorophosphazene) (PDCP) was prepared as previously described ${ }^{[12]}$. A pre-dried flask was loaded with $5.0 \mathrm{~g}(14.4 \mathrm{mmol})$ of hexachlorocyclotriphosphazene and aluminum chloride as a catalyst around 5 to $10 \mathrm{wt} \%$. The reactor was filled with high-purity nitrogen and heated to an initial temperature of $240-250{ }^{\circ} \mathrm{C}$. After the polymerization had proceeded for 3 hours, the reactor was cooled to $120{ }^{\circ} \mathrm{C}$, and $8 \mathrm{~mL}$ of diglyme were injected into the reactor to solubilize the crude product and to minimize cross-linking ${ }^{[13]}$. Aluminum chloride was removed by centrifugation $(7000 \mathrm{~g}$ at -10 $\left.{ }^{\circ} \mathrm{C}\right)$. The product identity was confirmed by ${ }^{31} \mathrm{P}-\mathrm{NMR}$ in a co-solvent of diglyme/deuterated chloroform (1:3). Subsequent nucleophilic substitution at the chlorine phosphorus centers ${ }^{[14]}$ was performed by transferring the product supernatant into a pre-dried flask containing $50 \mathrm{~mL}$ of anhydrous THF, where TEA and allylamine (both 3 eq. to chlorine) were added. The reaction was carried out on ice for 24 hours, and then, for additional 24 hours at room temperature. For purification, the mixture of raw products was filtered to remove the trimethylamine hydrochloride and the polymer was precipitated in a co-solvent (THF/water 1:2) first and then in pure water. The precipitated polymer, allylamino-poly(phosphazene) (AAPPZ), was collected and dried in vacuo. (Yield 4.61 g, 68 \%).

\subsection{Synthesis of cationic and anionic poly(phosphazene)s by thiol-ene reactions}

After synthesis of the precursor AAPPZ, we introduced two different amine side-chains, to provide cationic functionality, via reaction of the thiols of cysteamine and dimethyl(aminoethanethiol) with the alkenyl side-chains of the polymer. Analogous reactions to install various carboxylic acid 
groups were carried out using 3-mercaptopropionic acid (3MPA), 4-mercaptobutyric acid (4MBA), 6-mercaptohexanoic acid (6MHA) and 8-mercaptooctanoic acid (8MOA). Firstly, AAPPZ (100 mg, $1.27 \mathrm{mmol}$ of allyl groups) in $8 \mathrm{~mL}$ TFE was mixed with the designated mercaptoalkylamine or mercaptoalkyl carboxylic acid (3 eq. to allyl group) and flushed with nitrogen gas before adding 2,2dimethoxy-2-phenylacetophenone (0.05 eq. to allyl groups). The thiol-ene reaction was initiated under UV irradiation ( $\lambda=365 \mathrm{~nm}$ ) for 3 hours, and then the crude product was directly dialyzed against ultrapure water (molar mass cut-off $7 \mathrm{k} \mathrm{Da}$ ) until it became a clear solution. The product was recovered as a powder by freeze-drying. The polymers were analyzed by NMR and GPC.

\subsection{Formation of poly(phosphazene)-based complexes}

A bi-functional plasmid DNA (pDNA) coding for enhanced green fluorescent protein (EGFP) and luciferase from firefly Photinus pyralis was used throughout this work. In this pDNA, wild-type EGFP was modified to enhance the fluorescence excitation and emission at $488 \mathrm{~nm}$ and $507 \mathrm{~nm}$, respectively. Photinus pyralis luciferase was chosen as it emits yellow-green light with maximum at $560 \mathrm{~nm}$ across the $\mathrm{pH}$ range of 7.5-8.5.

Cationic poly(phosphazene)s with different side-chain ratios of cysteamine and DMAES were dissolved in $10 \mathrm{mM}$ HEPES pH 5.5. The anionic side-chain poly(phosphazene)s were dissolved in 10 mM HEPES pH 8.2. The pDNA was dissolved in ultrapure water. Complexation was performed under intense vortex mixing for $30 \mathrm{sec}$ at various ratios between the positively-charged amines of the polymer and the negative-charged phosphates on the pDNA (N/P ratio). In the case of the mixed cationic and anionic poly(phosphazene)s, the polycationic PPZ condensed both the anionic polymers and the pDNA in one step. These polymeric complexes were characterized by their stoichiometric composition ratio, in terms of positive amines $(\mathrm{N})$, negative carboxylates $(\mathrm{C})$ and negative DNA phosphates (P), i.e. N/C/P ratio.

\subsection{Characterization}

2.5.1 Nuclear Magnetic Resonance Spectroscopy (NMR) 
${ }^{1} \mathrm{H}$ - and ${ }^{31} \mathrm{P}-\mathrm{NMR}$ spectra were recorded on Bruker 400 and DRX-500 spectrometers, respectively. ${ }^{1} \mathrm{H}$ Diffusion-ordered spectroscopy $\left({ }^{1} \mathrm{H}-\mathrm{DOSY}\right)$ was recorded on Varian Inova $750(750$ $\mathrm{MHz}$ ). Solvents used were $\mathrm{CDCl}_{3}, \mathrm{D}_{2} \mathrm{O}, \mathrm{MeOD}$. All chemical shifts are reported in parts per million (ppm) relative to tetramethylsilane (TMS) or known solvent peak positions.

\subsubsection{Gel Permeation Chromatography (GPC)}

Molar masses of polymers were determined via gel permeation chromatography using a Shimadzu Prominence UPLC system fitted with a DGU-20A5 degasser, LC-20AD, CBM-20A LITE system controller, SIL-20A autosampler, CTO-20A oven and RID-10A refractive index detector. Separations were performed on a series of Aquagel 30-40-50 (300 x $7.8 \mathrm{~mm}, 5 \mathrm{~mm}$ bead size, Agilent UK) columns fitted with a matching guard column $(50 \times 7.8 \mathrm{~mm})$. The mobile phase was acidic buffer solution ( $1 \mathrm{M}$ acetic acid and $0.3 \mathrm{M} \mathrm{NaH}_{2} \mathrm{PO}_{4}$ ) at $1 \mathrm{~mL} \mathrm{~min}^{-1}$ flow rate and separations were performed at $37^{\circ} \mathrm{C}$. Column calibration was performed using Poly(2-vinylpyridine) Easi Vials (2 mL) standards (668 Da-211k Da, Polymer Standards Service, USA).

\subsubsection{Dynamic light scattering (DLS) and Zeta Potential Measurements}

Dynamic light-scattering and zeta potential measurements were performed using a Nanosizer ZS instrument (Malvern, UK) at $25^{\circ} \mathrm{C}$, equipped with a He-Ne laser $(\lambda=633 \mathrm{~nm})$ with a backscatter angle of $173^{\circ}$. For zeta potential, measurements were performed in $1 \mathrm{mM} \mathrm{KCl}$. Hydrodynamic radii distributions were calculated with Malvern software. From standard auto correlation functions, diffusion coefficients were related to particle hydrodynamic radius via the Stokes Equation:

$$
\left.\mathrm{R}_{\mathrm{H}}=\kappa_{\mathrm{B}} \mathrm{T} / 6 \pi \eta \mathrm{D} \quad \text { (Equation } 1\right)
$$

Where $\mathrm{R}_{\mathrm{H}}$ is the hydrodynamic radius, $\kappa_{\mathrm{B}}$ is the Boltzmann constant, $T$ is the temperature, and $\eta$ is the viscosity of the solvent. In addition, it was assumed that particles were spherical and noninteracting. 


\subsubsection{Electron Microscopy}

The morphologies of the polymeric complexes were characterized by transmission electron microscopy (JEOL JEM-2010) in negative staining mode using phosphotungstic acid (PTA, $2 \% \mathrm{w} / \mathrm{v}$ ). The complex suspensions $\left(10 \mu \mathrm{L}\right.$ of solutions at a plasmid concentration of $\left.62.5 \mu \mathrm{g} \mathrm{mL}^{-1}\right)$ were dropped onto a TEM grid (Carbon Film CF400, Electron Microscopy Sciences) and left for 5 min, when the residual sample was blotted away by capillary forces. Afterwards, the PTA solution was added to the grid for $1 \mathrm{~min}$, and then the grids were washed with ultrapure water and dried under lowpressure prior to TEM imaging.

\subsection{Potentiometric titration and gel electrophoresis}

The buffering capacities of cationic and anionic poly(phosphazene)s were determined by initial dissolution of the polymer in Milli-Q water to give a concentration of $50 \mathrm{mM}$ of side-chain units. The anionic polymer solutions $(0.5 \mathrm{~mL})$ were adjusted to $\mathrm{pH} 11$ with $1 \mathrm{~N} \mathrm{NaOH}$ and then titrated with 1 $\mathrm{N} \mathrm{HCl}$. In between each addition, the polymers were agitated under vortex for $30 \mathrm{sec}$ and allowed to stabilize for another $30 \mathrm{sec}$ prior to $\mathrm{pH}$ measurement. For cationic polymers, solutions of the same concentration were adjusted to $\mathrm{pH} 2$ with $1 \mathrm{~N} \mathrm{HCl}$, and then titrated with $1 \mathrm{~N} \mathrm{NaOH}$ using the same method used for the anionic polymers. pKa calculation and protonation degree $(\alpha)$ vs. $\mathrm{pH}$ were calculated from the potentiometric titration curves (more information on the calculations in SI).

For estimating the binding efficiency of pDNA complexation, gel electrophoresis was performed using $1 \%$ agarose containing $\left(0.5 \mu \mathrm{g} \mathrm{mL}^{-1}\right)$ ethidium bromide $(\mathrm{EtBr})$ at $110 \mathrm{~V}$ for $50 \mathrm{~min}$. Each sample was prepared to a $30 \mu \mathrm{L}$ volume containing $1 \mu \mathrm{g}$ plasmid. In heparin displacement tests, each polymer/DNA complex sample was challenged with different mass ratios of heparin to plasmid, using samples at a fixed volume $(30 \mu \mathrm{L})$ and incubated for 1 hour at $37^{\circ} \mathrm{C}$.

\subsection{Transfection, cytotoxicity, and confocal imaging on 2D monolayer}

For 2D transfection and cytotoxicity studies, U87MG cells were cultured in Dulbecco's Modified 
Eagle Medium (DMEM, Sigma) containing $10 \%$ fetal bovine serum, $2 \mathrm{mM}$ Glutamine and $100 \mathrm{mg}$ $\mathrm{L}^{-1}$ penicillin-streptomycin and maintained at $37{ }^{\circ} \mathrm{C}$ under a $5 \% \mathrm{CO}_{2}$ atmosphere. For $2 \mathrm{D}$ culture, the cells were seeded at a density of 25000 cells $\mathrm{cm}^{-2}$ in $24-$ well plates and incubated 24 hours prior to transfection. Then, polymer complexes were added at various pDNA concentrations in reduced serum media (OptiMEM) for 4 hours. The formulations were removed after the incubation, and cells were then cultured with fresh growth medium for additional 48 hours. Cell transfection was qualitatively assessed via fluorescence microscopy and quantified with Luciferase assay kits (from Promega). Cytotoxicity was evaluated by a MTT assay (Promega). Briefly, the transfected cells were treated with $10 \mu \mathrm{L}$ of $12 \mathrm{mM}$ MTT/PBS solution and incubated with $100 \mu \mathrm{L}$ phenol-red free media in each well for 4 hours. After the supernatant was removed, $50 \mu \mathrm{L}$ DMSO aliquots were added to the wells, mixed and incubated for $10 \mathrm{~min}$ before quantification on a plate reader with absorbance wavelength set at $540 \mathrm{~nm}$. For intracellular trafficking studies, U87MG cells were attached on poly-L-lysinecoated $8 \mu$-Slides (Ibidi) and treated with polymeric complexes carrying Cy5-labelled blank pDNA (prepared by Label $\mathrm{IT}^{\circledR}$ kit from Mirus) at the pDNA concentration of $0.3 \mu \mathrm{g}$ per well for 4 hours. Then the medium was changed to fresh growth medium for further incubation for another 20 hours. After this period, treated cells were incubated with $50 \mathrm{nM}$ of Lysotracker-green (Invitrogen) and $5 \mu \mathrm{g}$ $\mathrm{mL}^{-1}$ Hoechst 33342. Finally, confocal images (Leica SP5X) in live cells were taken. For tracking intracellular release of pDNA, CA-PPZ was labelled with cyanine 3 (Cy3) via conjugation with Cy3NHS (from GE) and then complexed with 6MHA-PPZ and Cy5-pDNA, forming Cy3-CAPPZ/6MHA-PPZ:Cy5-pDNA complexes. The preparation of confocal microscopic samples was by the same method described above.

\subsection{Transfection and tomographic scanning in a 3D spheroid model}

For establishing 3D spheroids of U87MG cells, ultra-low attachment 96-well round-bottom plates $\left(\right.$ Corning $^{\circledR}$ ) were seeded with 250-5000 U87MG cells per well in growth media, utilizing six replicates for each experimental condition. The plates were centrifuged at $200 \mathrm{rcf}$ for 5 min to induce cell accumulation and stimulate spheroid formation. After three days, the spheroids were adjudged 
ready for transfection studies. The spheroids were treated with different polymeric complexes and with different pDNA concentrations $(2$ and $4 \mu \mathrm{g} \mathrm{mL}-1)$ for 16 hours in OptiMEM and then incubated in growth media for another 80 hours. GFP expression in each spheroid was quantified at 96 hours post-treatment, via ImageJ (Fiji package) analysis directly using built-in histogram functions (Figure S9). The volume of the spheroids was calculated from phase-contrast microscopic images (Nikon Eclipse Ti) using an ImageJ plugin macro that measures spheroid area (A) from defined regions of interest (ROI). ${ }^{[15]}$ Then, the spheroid area was used to determine the radius $\left(\mathrm{R}=[\mathrm{A} / \pi]^{\wedge}(1 / 2)\right)$ and calculate the volume assuming that the cell aggregates formed equivalent spheres. To quantify metabolic activity in the spheroids, a resazurin reduction assay was performed. Briefly, the spheroids were treated with resazurin $(60 \mu \mathrm{M})$ in the growth media and incubated at $37^{\circ} \mathrm{C}$ for 4 hours. Following that, the 96-well plate was measured with a FlexStation II plate reader set at an excitation wavelength of $530 \mathrm{~nm}$ and at an emission of $590 \mathrm{~nm}$. The evaluation of membrane integrity in the cell was performed using 7AAD ( $5 \mu \mathrm{L}$ per well, $\left.50 \mu \mathrm{g} \mathrm{mL}^{-1}\right)$ and fluorescence observed in the mCherry channel (Ex 560/55 and filter 630/60) of the fluorescence microscope.

The quantification of fluorescence intensity in the spheroid model was carried out via selection of ROI and comparing histogram curves of fluorescence intensity (FI) for samples and the non-treated group control (Figure S9). GFP-positive counts were recorded when FI were higher than the maximum FI of non-treated groups, set as threshold (Equation 2). For GFP-gene transferred expression, there is no optimal positive control. For this reason, we quantified GFP expression via the percentage of GFP positive pixels. There were at least four replicates in each independent experiment and displayed data were analyzed using Prism software from four to nine independent experiments.

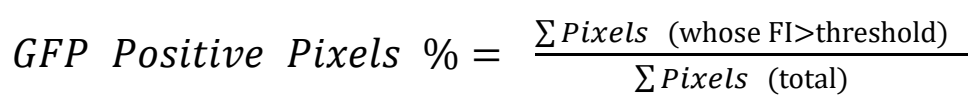

(Equation 2)

For 7AAD, the threshold is defined from the mean fluorescent intensity (Mean FI) in the untreated 
group as $0 \%$ and the mean FI of $0.5 \% \mathrm{v} / \mathrm{v}$ Triton X-100 treated group as $100 \%$. The percentages of 7AAD signals of treated spheroids are expressed as below.

$$
\begin{aligned}
& \text { Mean FI of 7AAD signals }=\frac{\text { Epixel } \times F I}{\text { PPixels }(\text { total })} \quad \text { (Equation 3) } \\
& 7 A A D \text { Positive } \%=\frac{(\text { Mean FI of treated spheroids })-(\text { Mean FI of non-treatment })}{\text { (Mean FI of TritonX treatment })-(\text { Mean FI of non-treatment })}
\end{aligned}
$$

For 3D imaging, spheroid samples were fixed with 4\% paraformaldehyde (PFA) first and their nuclei stained with DAPI. The fixed spheroids were loaded in appropriate glass capillaries with $1 \%$ w/v low-melt agarose. The imaging was performed by fluorescent light sheet microscopy FLSM (Light-sheet Z.1, Zeiss) and presented in the same fluorescence intensity scale using ImageJ software.

\subsection{GFP/Luciferase transfection in xenografted model}

All in vivo experiments were approved by the Research Ethics and Animal Welfare Committee at the Instituto de Salud Carlos III, Madrid, (PROEX 224/14) in agreement with the European Union and national directives. A subcutaneous xenograft model of GBM was established as previously described ${ }^{[16]}$. Briefly, athymic nude Foxn1 nu mice were subcutaneously injected with 1 million fresh U87MG cells in $80 \mu \mathrm{L}$ cultured media and $20 \mu \mathrm{L}$ Matrigel (BD), on both flanks. When tumor sizes reached around $100-150 \mathrm{~mm}^{3}$, polymer complexes with the GFP/Luciferase pDNA (16 $\mu \mathrm{g}$ per tumor) were injected intratumorally. One week later, before the mice were sacrificed, they were treated with Luciferin $50 \mathrm{mg} \mathrm{kg}^{-1}$ by IP injection. The transfected tumors were excised and imaged by an In Vivo Imaging System (IVIS $\left.{ }^{\circledR}\right)$.

\subsection{Down-regulation efficiency and clonogenic assays}

Complexes of polymers with siRNA against DYRK1A (siDYR) (ON-TARGETplus Human DYRK1A, Dharmacon) with siRNA concentration $1 \mu \mathrm{g} \mathrm{mL} \mathrm{m}^{-1}$ were added to U87MG (75K cells per 
well) in 12-well plates for 4 hours and then maintained in culture media for 24 hours. The silencing efficiency following transfection was then measured by qRT-PCR. The treated cells were collected for mRNA extraction by High-pure RNA isolation kit (from Roche) and finally mRNAs were trapped by a glass fiber fleece in filter tubes. After elution of mRNA, the reverse transcription of $200 \mathrm{ng}$ mRNA was performed by PrimeScript ${ }^{\mathrm{TM}}$ RT kit (from TaKaRa). The appropriate cDNA was mixed with SYBR-Green and either primers of DYRK1A (Forward 5'-3': GCAATTTCCTGCTCCTCTTG; Reverse 5'-3': TTACCCAAGGCTTGTTGTCC) or primers of HPRT (Forward 5'-3': TGACACTGGCAAAACAATGCA; Reverse 5'-3': GGTCCTTTTCACCAGCAAGCT), used as a reference (housekeeping gene). Gene expression was measured by quantitative RT-PCR (Lightcycler480 II, Roche).

For self-renewal assays, the silenced cells were detached and left in a serum-free medium, Neurobasal (Fisher-Scientific) supplemented with B27 (1:50), EGF (40 ng ml-1) and FGF (20 ng ml-

${ }^{1}$ ) (Peprotech) for three days to form spheroids. Then, the spheroids were dissociated by Accumax (Millipore) and the serum-deprived cells were seeded on 96-well plates (250 cells per well) for 7-10 days in the same serum-free medium. The numbers of secondary spheroids formed were counted.

\subsection{Combination treatment of siRNA of DYRK1A and temozolomide in xenograft models}

To investigate the therapeutic effectiveness of DYRK1A-silenced gene delivery in vivo, a subcutaneous xenograft model was established as described above. When tumor sizes reached around $100-150 \mathrm{~mm}^{3}$, the polymeric complexes were injected intratumorally with $4 \mu \mathrm{g}$ siDYR or the same amount of the scrambled sequence (siCtrl) for each tumor/day, over four consecutive days (total 16 $\mu \mathrm{g}$ siRNA for each tumor, $0.6 \mathrm{mg} / \mathrm{kg})^{[17]}$. On the same treatment days, mice from both study arms were also administered $5 \mathrm{mg} \mathrm{kg}^{-1} \mathrm{TMZ}$ by intraperitoneal injection (total dose $20 \mathrm{mg} \mathrm{kg}^{-1}$ each mouse). The tumor sizes were measured by a caliper and calculated by Volume $=\mathrm{W} \times \mathrm{W} \times \mathrm{L} / 2$, in which $\mathrm{W}$ represents width and $\mathrm{L}$ is length.

\subsection{Hemolysis tests}


Rat blood was collected in K2E tubes containing EDTA (BD Vacutainer ${ }^{\circledR}$ ) and was centrifuged at $1000 \mathrm{rcf}$ for $5 \mathrm{~min}$. After washing with PBS three times under the same centrifuge conditions, the red blood cells were re-suspended either in PBS 7.4 or in PBS 5.5 at $3 \%(\mathrm{w} / \mathrm{v})$ concentration. The erythrocyte suspensions were allocated across 96 wells and incubated with either CA-PPZ:pDNA or CA-PPZ/6MHA-PPZ:pDNA complexes for 2 hours at $37{ }^{\circ} \mathrm{C}$ under mild shaking. Then, the plates were centrifuged and the supernatants transferred to a new plate for colorimetric analysis. The absorption of the released hemoglobin was measured in a 96-well plate reader at an excitation wavelength of $570 \mathrm{~nm}$. The maximum hemolysis (positive control) was established using cells treated with $1 \%(\mathrm{v} / \mathrm{v})$ Triton $\mathrm{X}-100$ and the minimum value was set by treatment with PBS either at $\mathrm{pH} 7.4$ or at $\mathrm{pH} 5.5$.

\subsection{Statistical Analysis}

All data are presented as mean \pm standard error of the mean (SEM). For statistical analysis, data was transformed if required for normalization. Normality of the data was tested by a Shapiro-Wilk test. Significant differences where tested with student-t tests or ANOVA. $P$ values $<0.05$ were considered to be significant, although $\mathrm{P}$ values below this level are also noted in individual figures and in the text. Statistical tests applied and the number of independent replicates is noted in the figure captions. All statistical analyses were carried out via GraphPad Prism 7.

\section{Results}

The strategy for the synthesis of various substituted poly(phosphazene)s was via thermal ringopening polymerization of the cyclic monomer hexachlorophosphazene (1) and reaction of the resultant poly(dichlorophosphazene) with allylamine under basic conditions to afford vinylic sidechains ${ }^{[6 b]}$. These were amenable to thiol-ene derivatization to yield a series of polycations and polyanions (Figure 1a). 



Figure 1. Synthesis and characterization of cationic and anionic poly(phosphazene)s. a) Synthesis of either cationic or anionic poly(phosphazene)s via thermal ring-opening polymerization, nucleophilic substitution and thiol-ene click chemistry. (b, c) Protonation degree $(\alpha)$ of different cationic poly(phosphazene)s (b) and of different anionic poly(phosphazene)s (c) against $\mathrm{pH}$.

The initially formed poly(dichlorophosphazene) (2) was characterized by ${ }^{31} \mathrm{P}-\mathrm{NMR}$ (Figure S1), and this compound was reacted by nucleophilic substitution at the $\mathrm{P}-\mathrm{Cl}$ bonds with allylamine to generate the key precursor polymer, allylamino-polyphosphazene (AAPPZ). A range of different cationic (4-6) and anionic (7-10) side-chain polymers (Figure 1a) were generated from AAPPZ via thiol-ene click chemistry. Reactions proceeded to a high degree ( $\geq 93 \%$ substitution of the allyl groups by the cationic side-chains) and good overall yield ( $\geq 71 \%$ ). Product identity was confirmed by ${ }^{1} \mathrm{H}-$ NMR (Figures S2). The molar masses of the final product cysteamine-linked poly(phosphazene) (CA-PPZ, 4) were estimated by aqueous GPC, with calibration against commercial standards of 
poly(2-vinylpyridine). Two families of CA-PPZ polymers were characterized with molar masses of Mw 35.9 $\pm 3.9 \mathrm{kDa}(\mathrm{PI}=1.54)$ and $26.0 \pm 2.4 \mathrm{kDa}(\mathrm{PI}=1.56)$, respectively.

The polymers were intended as nucleic acid carriers, for which their abilities to act as 'protonsponges' and/or membrane disruptors ${ }^{[18]}$ are highly dependent on their ability to accept protons and to change their hydrophilic/ lipophilic balance across intracellular $\mathrm{pH}$ ranges. The apparent $\mathrm{pKa}$ values of the polycations and polyanions were thus determined by potentiometric titrations (Figure S3 and Figure 1 b, c). As expected, the amine side-chain polymers remained protonated across cytosolic and endolysosomal $\mathrm{pH}$ ranges. However, the various aliphatic acid side-chain polymers, derived from 3-mercaptopropanoic acid (3MPA), 4-mercaptobutanoic acid (4MBA), 6mercaptohexanoic acid (6MHA), and 8-mercaptooctanoic acid (8MOA) exhibited buffering regions across the range of $\mathrm{pH} 5$ to $\mathrm{pH}$ 8. These titration curves were converted to line charts plotting protonation degree $(\alpha)$ against $\mathrm{pH}$ (methods in SI- Appendix). The largest shift in protonation over the critical range of physiological $\mathrm{pH}$ to early endosomal $\mathrm{pH}$ ( $\mathrm{pH} 7.4$ to 6.0) was exhibited by 6MHAPPZ with a change in $\alpha$ of $61.9 \pm 3.6 \%$. This was significantly higher than the other anionic polymers (Table S1) and suggested that this specific polymer should be the most advantageous in enhancing endosomal escape of the gene carriers.

The cationic polymers were screened for polyelectrolyte complex formation with plasmid DNA (pDNA) at varying N/P ratios (Figure S4). At N/P = 8, the diameters of the complexes were all close to $80 \mathrm{~nm}$ and the zeta potentials close to $+40 \mathrm{mV}$. When the pDNA was added to CA-PPZ in the presence of the various polyanions (at an 8:4 ratio of amines to carboxylic acids in the mixture), the diameters increased to $\sim 100 \mathrm{~nm}$ and the surface charges reduced to approximately $+30 \mathrm{mV}$. In addition, although the size of the complexes did not increase significantly with the addition of increasing amounts of the polyanion 6MHA-PPZ, the total derived count rate in the light scattering experiments increased over one order of magnitude compared to the same complexes without this anionic PPZ (Figure S4c), suggesting that more compact particulate complexes were formed in the presence of polyanionic PPZs. This was supported also by TEM images showing that CA-PPZ:pDNA displayed looser structures than CA-PPZ/6MHA-PPZ:pDNA complexes (Figure 2c, d). TEM images 
also corroborated the measurements made by photon correlation spectroscopy and confirmed that CA-PPZ:pDNA complexes were smaller than CA-PPZ/6MHA-PPZ:pDNA complexes. Polymer/pDNA complexes were isolated from non-complexed species by high-speed centrifugation (20K rcf, $60 \mathrm{~min}$ ) and the production yields for CA-PPZ:pDNA and CA-PPZ/6MHA-PPZ:pDNA complexes were calculated as $85 \pm 8 \%$ and $97 \pm 2 \%$, respectively.

Agarose gel electrophoresis experiments were performed in the absence and presence of a competing polyanion, heparin, to test the ability of the different polycationic PPZs for retaining pDNA in the form of polyelectrolyte complexes. The data indicated that progressive replacement of the primary-amine CA-PPZ with tertiary-amine PPZ reduced the complexation capacity with pDNA (Figure 2a) as shown by increased pDNA migration from the complexes with PPZs substituted with $50 \%$, and particularly $100 \%$ tertiary amine groups. Complexes of CA-PPZ:pDNA and CAPPZ/6MHA-PPZ:pDNA were also analyzed by agarose gel electrophoresis and then challenged with heparin under simulated endosomal $(\mathrm{pH} \mathrm{5)}$ and standard physiological ( $\mathrm{pH} 7.4)$ conditions (Figure 2b). There was less pDNA release from both CA-PPZ:pDNA and CA-PPZ/6MHA-PPZ:pDNA complexes at $\mathrm{pH} 5$ than at $\mathrm{pH} 7$, likely due to increased polymer protonation in the more acidic environment. Also, we observed that CA-PPZ associated more strongly with pDNA than the mixed CA-PPZ/6MHA-PPZ system since the latter showed more visible gel loading bands and partial pDNA migration at low heparin incubation ratios. This effect was particularly notable at $\mathrm{pH} 7.4$, suggesting that 6MHA-PPZ might interact with CA-PPZ and modulate pDNA binding, especially when the 6MHA polymer became fully charged at its carboxylate functional groups.

Complexes based on cationic PPZs were initially tested for their ability to transfect U87MG glioblastoma cells when cultured as adherent monolayers. Cysteamine (primary amine) functionalized PPZs were more effective in inducing gene expression as compared to DMAES (tertiary amine side-chain) PPZs. This was likely caused by the high pDNA-binding efficiency of primary amine-poly(phosphazene) at the low N/P ratio of 8:1 (Figure 2a). In these experiments, the most favorable compromise between transfection efficiency and cell viability occurred at 8:1 N/P ratio with primary amine-PPZ:pDNA, and with the polymer of higher molecular weight (Mw 36k 
Da) (Figure S5). In order to provide for an endosomal escape mechanism, we prepared CAPPZ:pDNA complexes which also contained polyanionic PPZs (3MPA-, 4MBA-, 6MHA-, or 8MOAPPZ) in order to introduce aliphatic carboxylate side-chains with different ionization capacity at different $\mathrm{pH}$ ranges. Luciferase expression assays were run in U87MG monolayers using these complexes as gene carriers, and with PEI:pDNA complexes at the same N/P ratios as a positive control (Figure 2e). The CA-PPZ/6MHA-PPZ:pDNA complexes showed the highest level of transgene expression combined with a minimal effect on cell metabolic activity as compared to the CA-PPZ:pDNA complexes. Similar enhancements in transfection were not observed in mixed complexes containing other polyanionic PPZs, indicating polymer-specific effects that we attribute to the significant change in protonation of 6MHA-PPZ at endosomal $\mathrm{pH}$ and in de-protonation at cytosol $\mathrm{pH}$ for $\mathrm{pDNA}$ release, especially across the early endosomal range ( $\mathrm{pH} 7.4-6.0$ ). In addition, all polyanionic PPZs reduced the cytotoxicity of the complexes, which was likely due to partial shielding of the cationic charges of CA-PPZ. The transfection enhancing effect of 6MHA-PPZ was also effective with other cationic polymers with similar structure such as poly-L-lysine (PLL), and translated to other clinically relevant glioblastoma models such as primary GBM1 cells ${ }^{[19]}$ with remarkable efficacy (Figure S6). We selected 6HMA-PPZ for nanocarrier modification based on its unique ability to enhance gene transfection, a characteristic probably linked to the $\mathrm{pKa}$ and hydrophilic/hydrophobic balance of this polymer. The other polyanionic polymers, 3MPA-, 4MBAand 8MOA-PPZ, were accordingly not taken forward for the rest of this study. 
a)

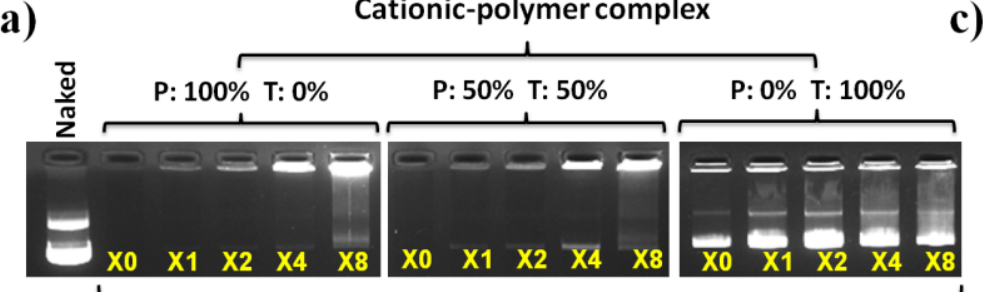

Weight ratio of Heparin to pDNA

b)

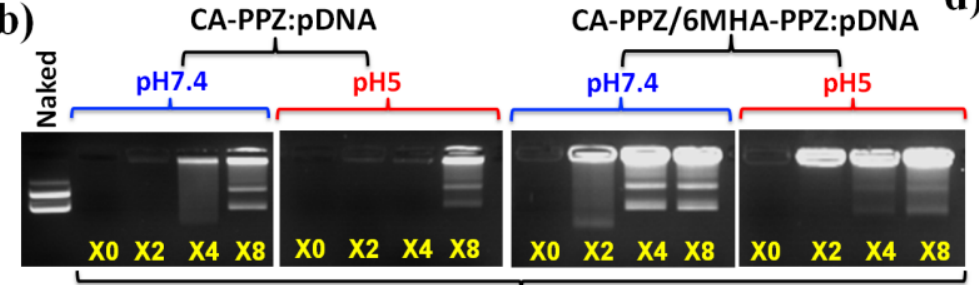

Weight ratio of Heparin to pDNA c)

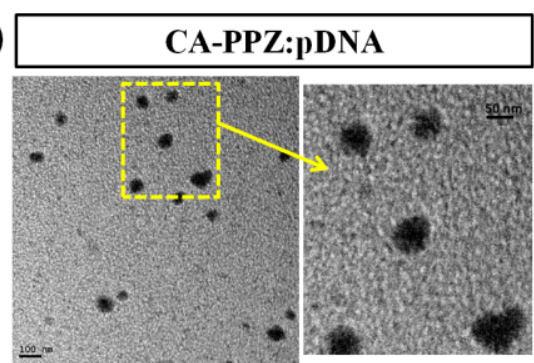

d)

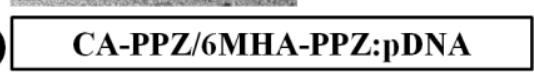

e)

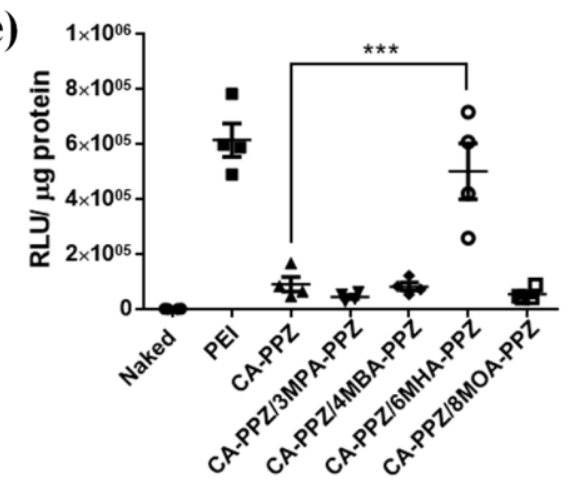

f)

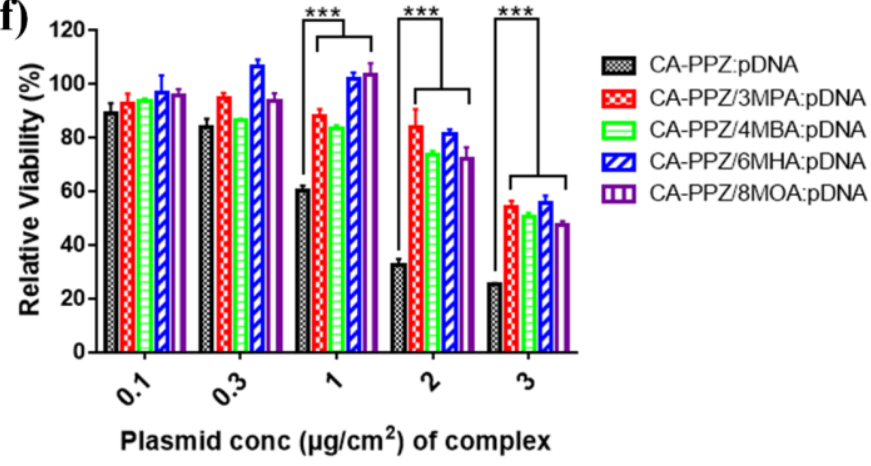

Figure 2. Design of optimized PPZ:pDNA complexes. (a) Gel retardation and heparin displacement tests of complexes prepared with PPZs having 100\% primary amines (P100\%), P50\% or P0\% (8:1 $\mathrm{N} / \mathrm{P}$ ratio). The remaining substitutes of each polymer correspond to tertiary amines: $\mathrm{T} 0 \%, \mathrm{~T} 50 \%$ and T100\%, respectively. (b) Gel electrophoresis and heparin-displacement tests of CA-PPZ:pDNA complexes and CA-PPZ/6MHA-PPZ:pDNA complexes (8:4:1 N/C/P ratio) at $\mathrm{pH} 7.4$ and $\mathrm{pH} 5$. (c, d) TEM images of CA-PPZ:pDNA and CA-PPZ/6MHA-PPZ:pDNA complexes. (e) Luciferase

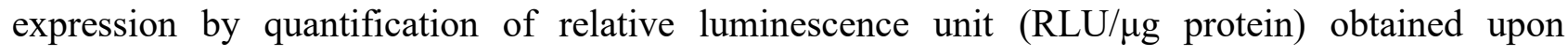
transfection of U87MG cells with CA-PPZ:pDNA complexes (8:1 N/P ratio) and different CAPPZ/polyanionic PPZ:pDNA complexes (N/C/P ratio of 8:4:1, pDNA dose $1 \mu \mathrm{g} \mathrm{cm}^{-2}$ ) in OptiMEM ( $\mathrm{n} \geq 4$ ). (f) Cell metabolic activity at $48 \mathrm{~h}$ post-treatment with different concentrations of the same complexes $(\mathrm{n}=6) . * * * p$ value $<0.01$ via one-way ANOVA analysis with Dunnett's multiple comparisons test with a single pooled variance. 
To understand the performance of CA-PPZ and CA-PPZ/6MHA-PPZ complexes, internalization and intracellular trafficking were investigated by flow cytometry and confocal microscopy. CAPPZ:pDNA and CA-PPZ/6MHA-PPZ:pDNA complexes were prepared with Cy5-labelled pDNA and incubated with U87MG cells under the same transfection conditions described previously. Flow cytometry (Figure 3a) and confocal micrographs (Figure 3b) indicated similar levels of cellular uptake for both prototypes. However, there were significant differences in the intracellular distribution of Cy5-labeled pDNA in relation to a late endosome/lysosome marker (Lysotracker, Invitrogen) between both prototypes. At $24 \mathrm{~h}$ post-treatment, the Cy5-labelled pDNA complexed with CA-PPZ/6MHA-PPZ was more broadly distributed in the cytosol, and distal to the green-stained lysosomes. CA-PPZ:pDNA complexes remained inside lysosomes, resulting in yellow signals in the confocal merged image (Figure 3b). The analysis of fluorescence intensities in the confocal images at $24 \mathrm{~h}$ post-treatment indicated that the co-localization ratios (Manders' overlap coefficient as defined using the ImageJ program) of Cy5-pDNA to lysosomal markers were $0.53 \pm 0.11$ in CA-PPZ complexes and $0.28 \pm 0.08$ in CA-PPZ/6MHA-PPZ complexes, respectively, with a $p$ value of 0.013 via Student's T-test. These data confirmed that the addition of 6MHA-PPZ facilitated the escape of the complexes from the endosomal compartment. We hypothesized that these results might be related to a capacity of 6MHA-PPZ for destabilizing biological membranes at endosomal $\mathrm{pH}$. To check this hypothesis, hemolysis tests were performed at different $\mathrm{pH}$ values. It was found that CA-PPZ/6MHAPPZ:pDNA complexes had no marked hemolytic effects at pH 7.4, in contrast to CA-PPZ:pDNA complexes and the internal control (PLL complexes). In contrast, CA-PPZ/6MHA-PPZ:pDNA complexes showed a clear hemolytic effect similar to those observed for PLL complexes at pH 5.5. Therefore, these experiments confirmed that CA-PPZ/6MHA-PPZ:pDNA complexes were the only systems tested which displayed $\mathrm{pH}$-dependent membrane permeating properties, and the data further suggested that membrane disruption would be triggered in the endosomal compartments (Figure S7). In addition to endosomal escape, intracellular distributions of the polycations and pDNA were investigated at $24 \mathrm{~h}$ and $48 \mathrm{~h}$ post-treatment for both complexes to further understand pDNA release from the nanocarriers. It was observed that Cy5-labelled pDNA (red signal) was separated from CA- 
PPZ/6MHA-PPZ complexes (co-localized yellow), particularly after 48h (Figure 3c and d). On the other hand, the CA-PPZ complex retained its cargo pDNA to a much higher extent, which may be the reason why the single polycation formulations demonstrated lower transgene expression.

a)
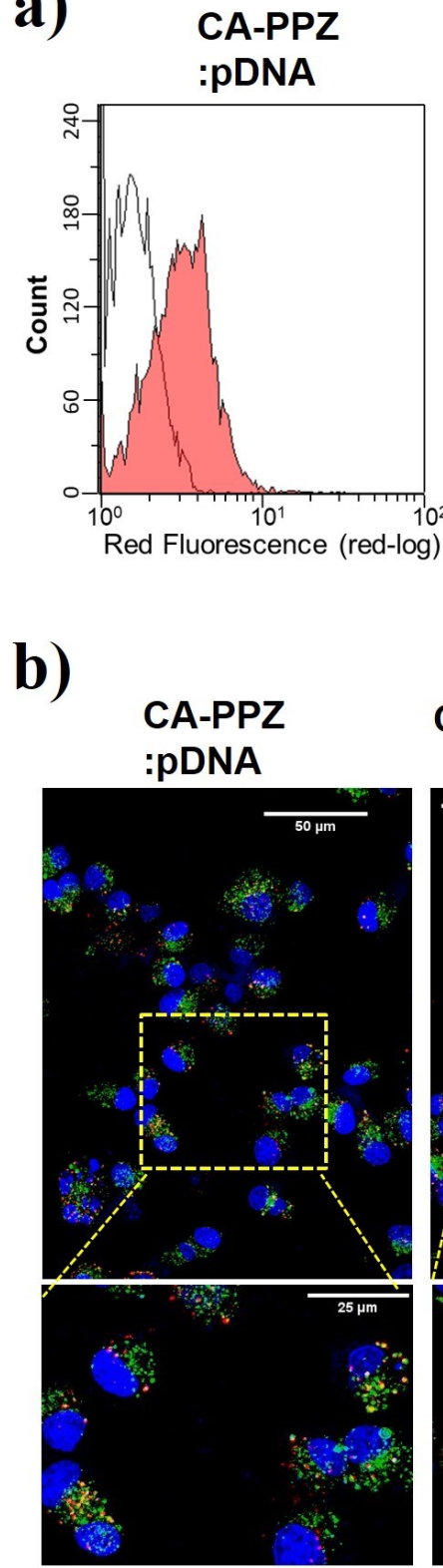

CA-PPZ/6MHA-PPZ :pDNA

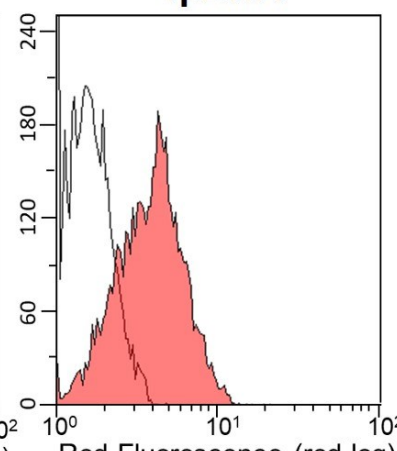

Red Fluorescence (red-log) c)
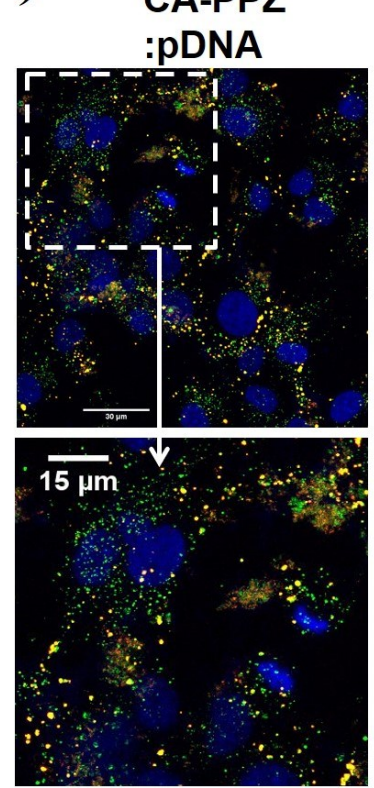

CA-PPZ/6MHA-PPZ

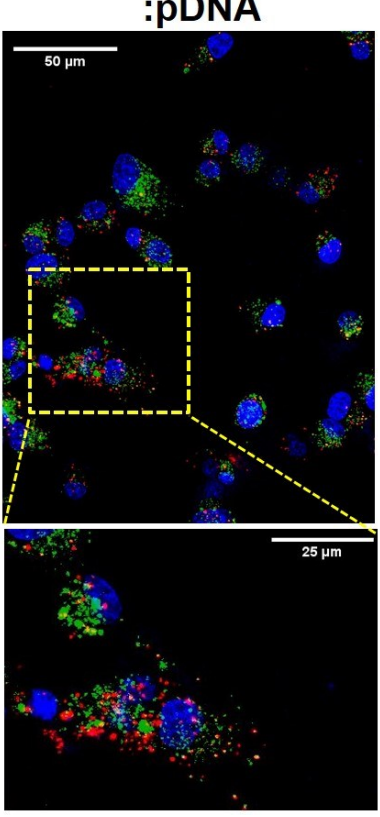

d)
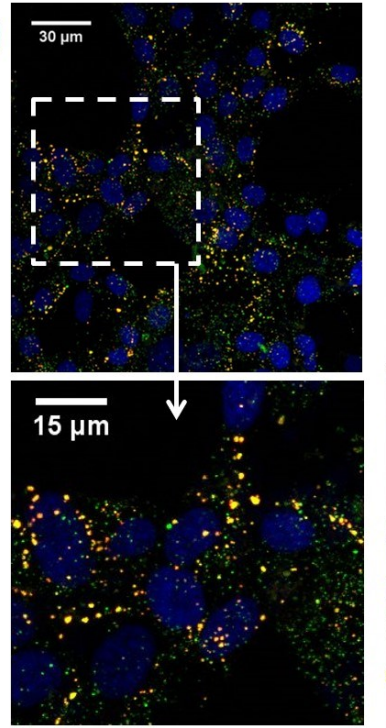

CA-PPZ/6MHA-PPZ :pDNA
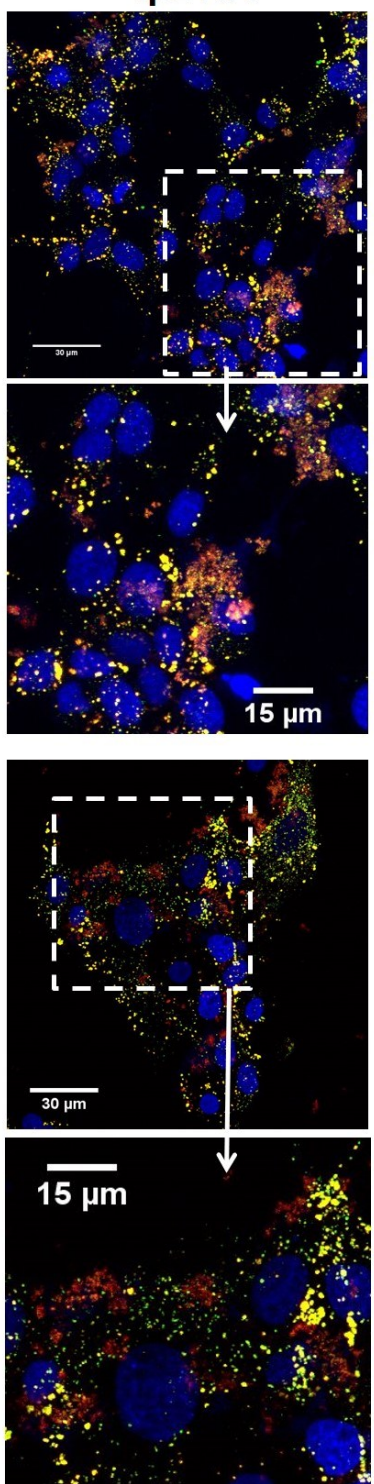

Figure 3. Comparison of the intracellular trafficking of two selected PPZ prototypes. a) Internalization of both complexes carrying Cy5-labeled pDNA after $4 \mathrm{~h}$ incubation with U87MG cells measured by Fluorescence Activated Cell Sorting. b) Intracellular distribution after 24 h of Cy5labeled pDNA (red) and a late-endosome/lysosome compartment marker (Lysotracker, in green) upon 
delivery of CA-PPZ:pDNA or CA-PPZ/6MHA-PPZ:pDNA complexes. Intracellular distribution of Cy3-CA-PPZ (green) and Cy5-pDNA (red) after $24 \mathrm{~h}$ (c) and $48 \mathrm{~h}$ (d) upon delivery of CAPPZ:pDNA or CA-PPZ/6MHA-PPZ:pDNA complexes.

Following demonstration of transgene expression in 2D monolayers, we established threedimensional spheroids of U87MG cells as simple mimics of residual tumor regions around glioblastoma-margin areas after surgery or radiotherapy ${ }^{[20]}$. Considering the limited penetration of excitation in microscopes and in order to exclude complicating factors of necrosis inside the spheroids, we selected small spheroids (diameter $\sim 250 \mu \mathrm{m}$ ) as our model. PPZ:pDNA complexes were assayed initially to evaluate any inherent toxicity by measuring spheroid size and metabolism through phasecontrast images and resazurin reduction assays (Alamar Blue), following the methods previously described $^{[15,21]}$ (Figure S8). Spheroids treated only with pDNA reduced slightly in volume after 16 hours in OptiMEM media, most likely due to some nutrient depletion over this period. Incubation of the spheroids with the CA-PPZ:pDNA complexes at $4 \mu \mathrm{g} \mathrm{mL}^{-1}$ caused a significant decline in volume to $87 \pm 3 \%$ of the initial value $(P<0.004)$ at 96 h-post treatment, whereas the analogous CAPPZ/6MHA-PPZ:pDNA complexes did not cause statistically significant reduction $(P=0.132)$ at the same dose. In resazurin reduction assays, the metabolic activities of CA-PPZ/6MHA-PPZ:pDNA treated spheroids were slightly higher than the untreated spheroids, whereas CA-PPZ:pDNA treated spheroids at both DNA doses $\left(2 \mu \mathrm{g} \mathrm{mL}^{-1}\right.$ and $\left.4 \mu \mathrm{g} \mathrm{mL} L^{-1}\right)$ showed no significant difference in metabolic activity compared to un-treated spheroids. These experiments indicated some possible minor toxicity for CA-PPZ:pDNA at the highest dose, and, importantly, no signs of toxicity for CA-PPZ/6MHAPPZ:pDNA complexes within the dose-range tested.

Further tolerability assays were performed monitoring cell membrane damage with 7Aminoactinomycin D (7AAD) on the previously used spheroid model. These experiments were performed in parallel with transgene expression level assays of Green Fluorescent Protein (GFP) upon pDNA delivery with different polymer complexes (Figure 4). CA-PPZ/6MHA-PPZ successfully 
delivered pDNA encoding GFP as demonstrated by the percentage of GFP-positive pixels at the regions of interest (ROIs) of spheroid images $(56 \pm 8 \%$ and $89 \pm 4 \%$ for pDNA doses of 2 and $4 \mu \mathrm{g}$ $\mathrm{mL}^{-1}$, respectively). These GFP levels were significantly higher than those obtained with CAPPZ:pDNA and PEI:pDNA either in the fluorescent images or the quantified analysis. Regarding 7AAD staining, there were no statistically significant differences between the polymeric complexes at the same dose, and the levels were always far below those of the positive control Triton X-100. In summary, the spheroid model experiments suggested that CA-PPZ/6MHA-PPZ:pDNA complexes achieved better transfection/toxicity ratios than CA-PPZ:pDNA as well as the widely-used PEI:pDNA system.

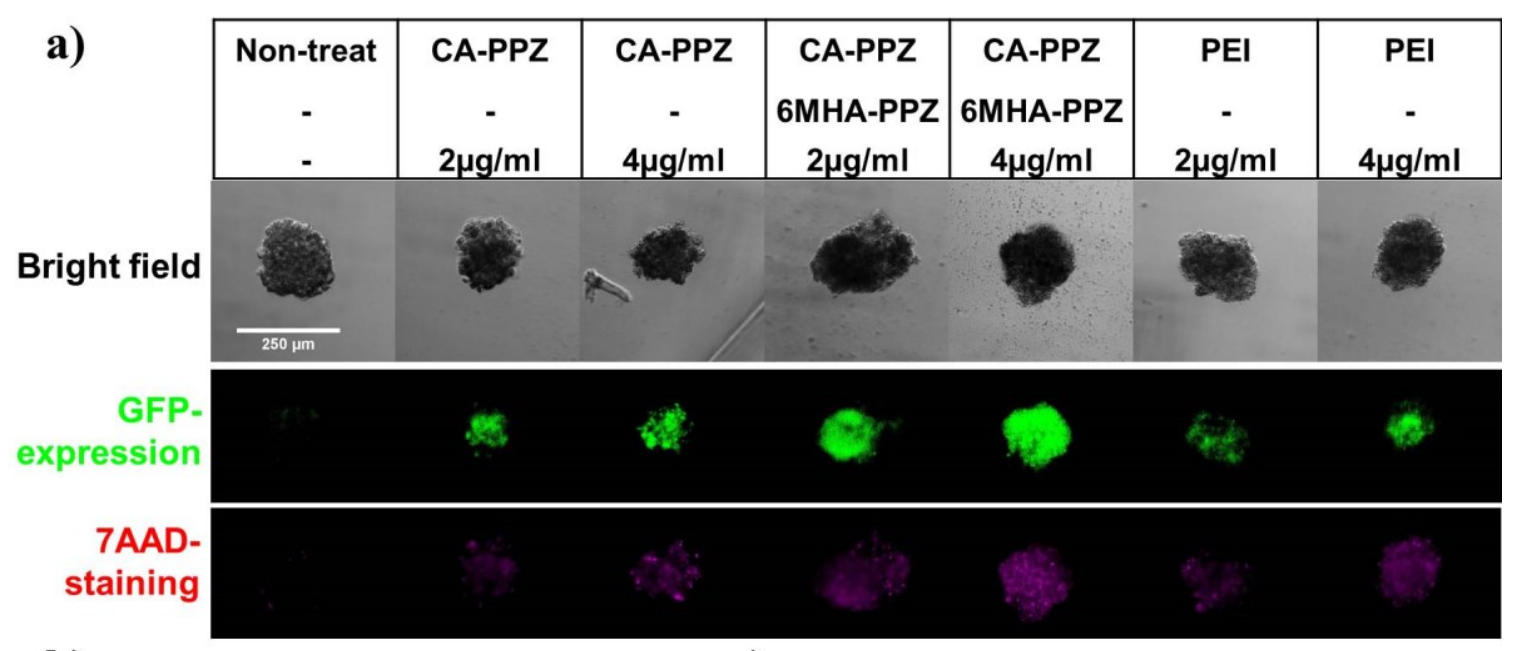

b)
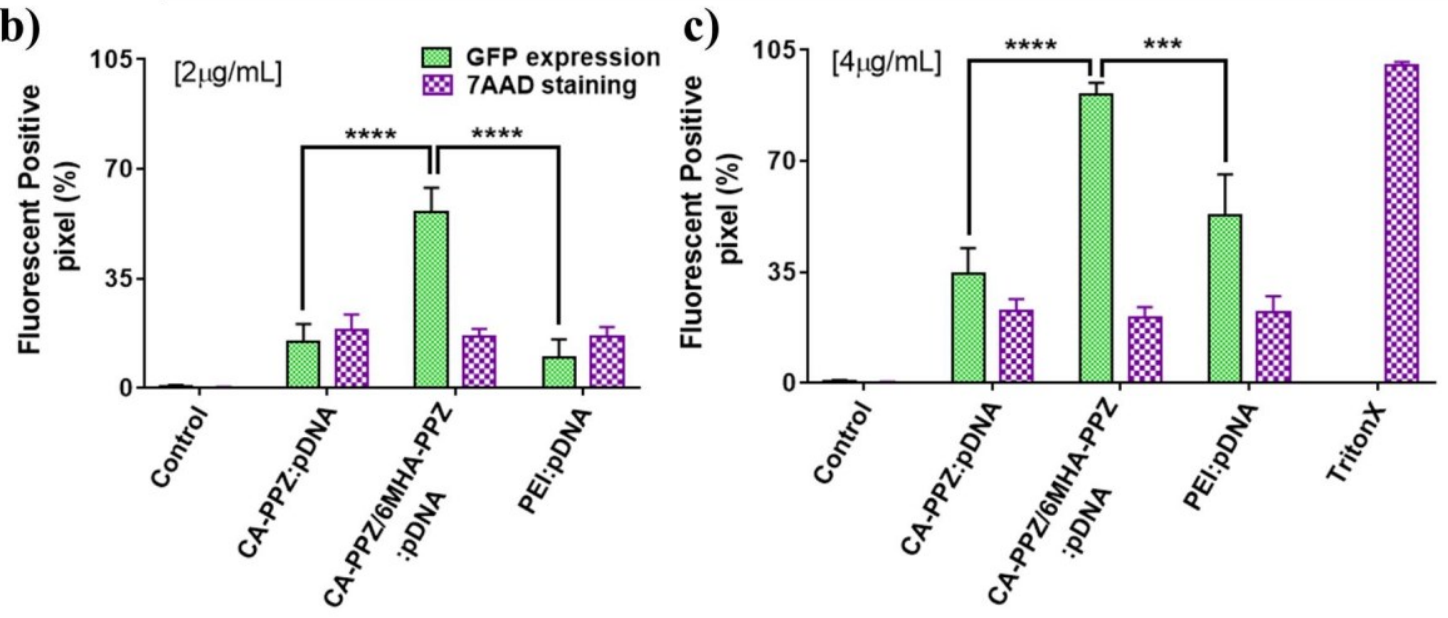

Figure 4. Transfection efficacy and viability of U87MG spheroids treated with polymeric complexes at two pDNA doses in OptiMEM. a) Overlay microscopy images showing GFPexpression and 7AAD-staining of U87MG-spheroids after treatments with CA-PPZ:pDNA (N/P 8:1) 
and CA-PPZ/6MHA-PPZ:pDNA (N/C/P 8:4:1) at the pDNA doses of $2 \mu \mathrm{g} \mathrm{mL}^{-1}$ and $4 \mu \mathrm{g} \mathrm{mL}^{-1}$. b, c) Quantification of GFP-fluorescence and 7AAD signals from the ROIs of spheroids in four independent experiments; spheroids treated with $2 \mu \mathrm{g} \mathrm{mL} L^{-1}$ pDNA are analyzed in (b), those treated with $4 \mu \mathrm{g} \mathrm{mL}^{-1}$ in (c). $(* * * *$ represents $p<0.001$ and $* * *$ is $p<0.01$, analyzed by a one-way ANOVA with a Dunnett's multiple comparison test with a single pooled variance; $n=4-6$ ).

To obtain further insight into the delivery system penetration and gene transfer throughout the spheroids, fluorescence tomographic scanning was performed by fluorescent light-sheet microscopy (FLSM) (Figure 5; tomographic-scanning videos in supplementary information). When the spheroids were treated with complexes at the highest pDNA dose $\left(4 \mu \mathrm{g} \mathrm{mL}^{-1}\right)$ homogeneous GFP transfection was apparent even in the core parts of the spheroids treated with CA-PPZ:pDNA and CAPPZ/6MHA-PPZ:pDNA, although GFP fluorescence was most intense with the latter system. At the lowest pDNA dose $\left(2 \mu \mathrm{g} \mathrm{mL} \mathrm{mL}^{-1}\right)$ of CA-PPZ/6MHA-PPZ:pDNA complexes still provided consistent transfection throughout all the spheroid, whereas transfection with CA-PPZ and PEI:pDNA complexes was clearly less (Figure 5a). These data suggested a more efficient gene delivery for CAPPZ/6MHA-PPZ:pDNA as compared to CA-PPZ:pDNA and the laboratory transfection standard PEI:pDNA system in this 3D model. The results also indicated that the mixed polycations/polyanion/nucleic acid system had a high capacity to penetrate through a 3D tumor structures of $\sim 250 \mu \mathrm{m}$ in diameter. Maximum intensity projections (Figure 5b) indicated strong fluorescence throughout the spheroids transfected with the CA-PPZ/6MHA-PPZ:pDNA complexes, supporting our previous observations. Gene expression was also evaluated in larger spheroids (diameter $\geq 400 \mu \mathrm{m}$ ) where we found less transfection in the spheroid cores as compared to those tested previously (Figure S10). This could be explained by two arguments. Firstly, the nanocarriers were unable to transport to the tumor core due to the extended distance. Secondly, the tumor cells in the inner core of these large tumors may have been necrotic due to excessively low oxygen tension and thus be unable to produce GFP. 
a)

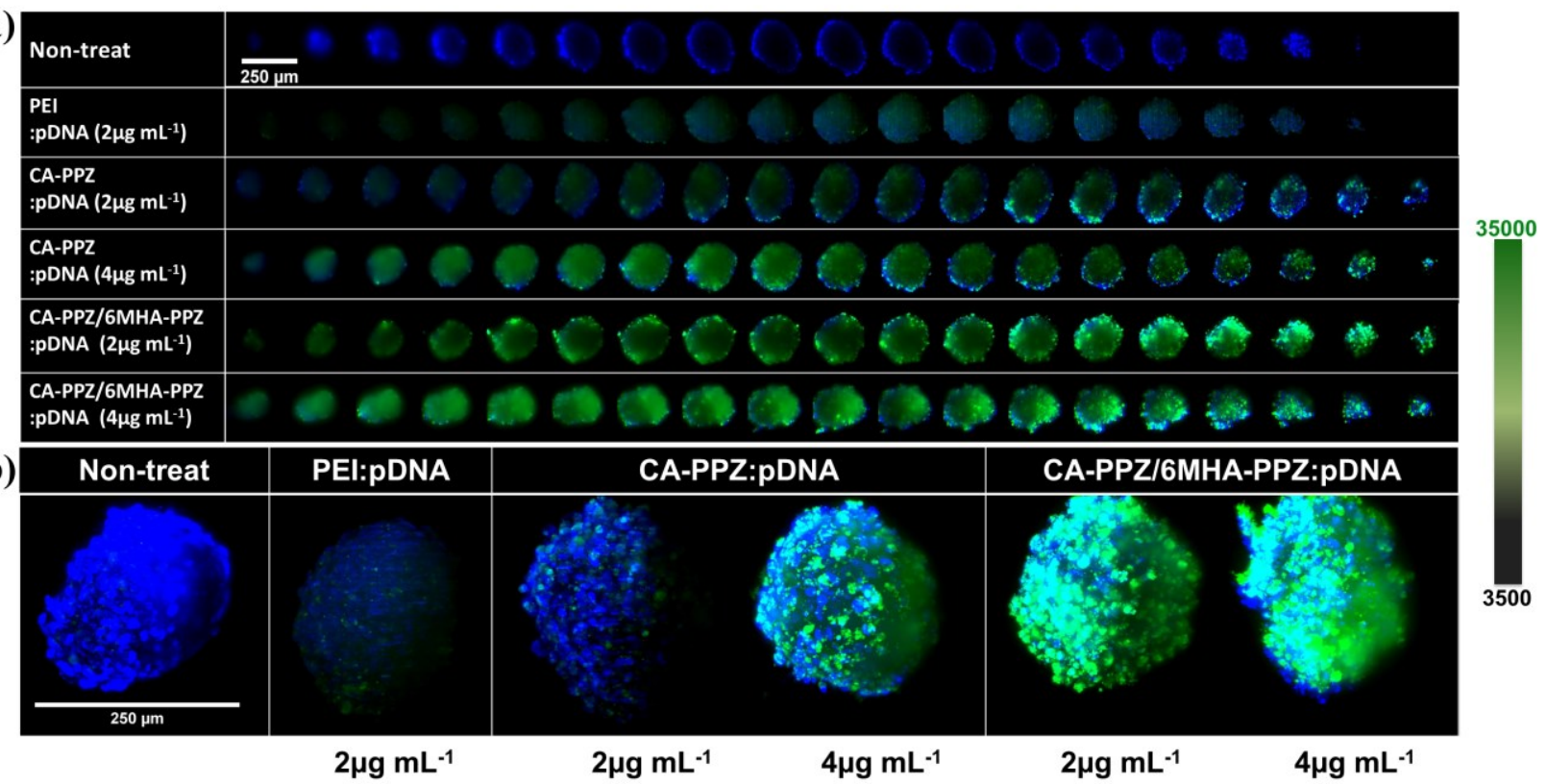

Figure 5. 3D tomographic images of GFP-transfected spheroids taken by FLSM. (a) 3D section images of CA-PPZ:pDNA and CA-PPZ/6MHA-PPZ:pDNA treated spheroids (diameter $250 \mu \mathrm{m}$ ) at two pDNA doses of 2 and $4 \mu \mathrm{g} \mathrm{mL}^{-1}$. (b) Maximum intensity projections along Z-axis (XY projection) images of spheroids treated with different polymer:pDNA complexes at two doses. The images were taken by FLSM. Blue corresponds to DAPI staining; green to GFP fluorescence. The fluorescence images were analyzed by ImageJ and processed under the same scale of fluorescence intensity.

We next evaluated gene-transfer efficiency of the PPZ:nucleic acid complexes in centimeterscale xenografted tumors in vivo. Nude mice were subcutaneously implanted with U87MG cells on the flanks, and when the tumors attained a size of $100-150 \mathrm{~mm}^{3}$, polymeric complexes with the bifunctional plasmid (encoding GFP and Luciferase) were injected intra-tumorally (16 $\mu \mathrm{g}$ pDNA for each tumor). At the end of the experiment, tumors were excised, sectioned and observed by IVIS. The data indicated that the tumors were transfected by all the polymeric complexes tested, with a marked increase in luminescence compared to un-treated tumors (Figure 6a, b). ROI quantification of luciferase expression showed that CA-PPZ/6MHA-PPZ:pDNA complexes were similar in transfection efficacy to PEI-complexes and around 1.5-fold more effective than CA-PPZ:pDNA 
complexes (Figure 6b).
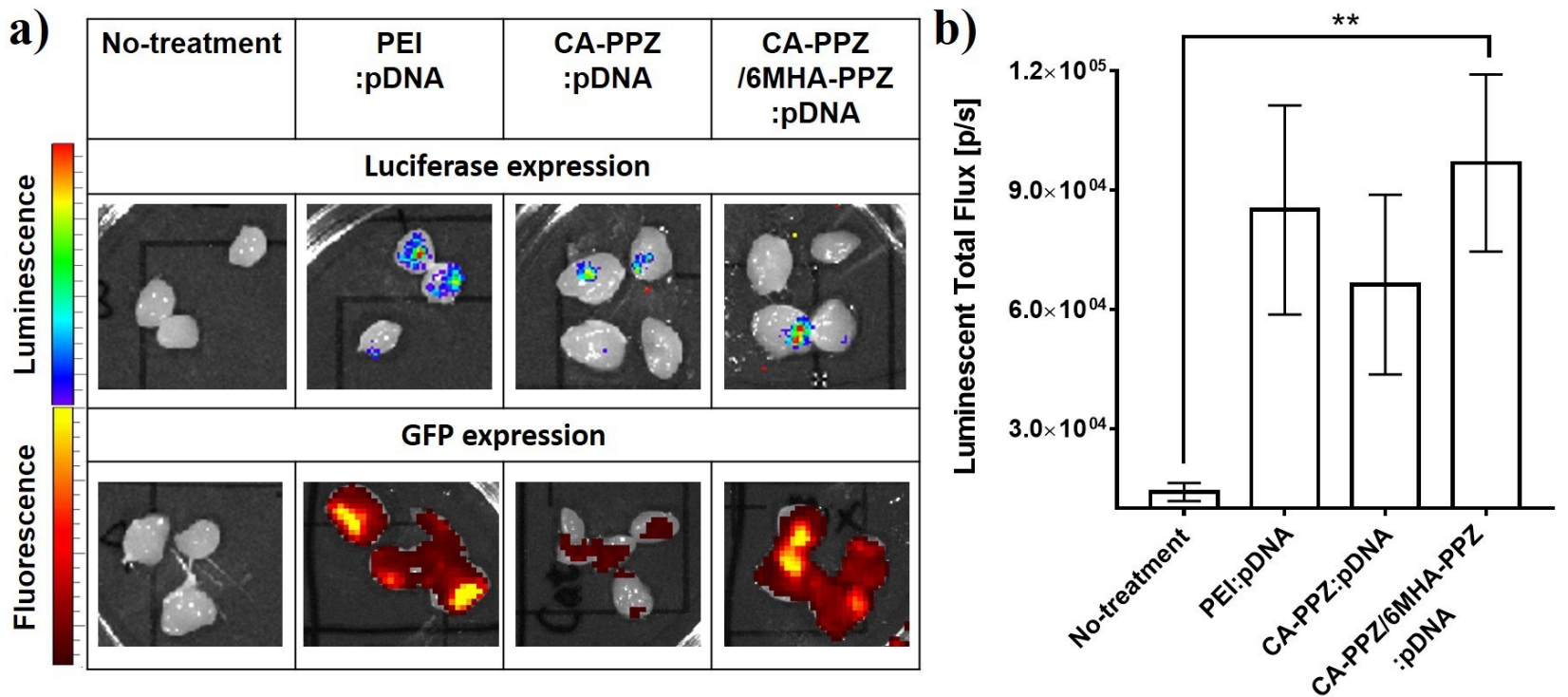

Figure 6. Transfection of polymeric complexes on xenograft tumors of U87MG. a) IVIS images of luciferase/GFP transfected U87MG tumors in nude mice. b) Quantitative analysis of the fluorescence in the ROI. ${ }^{* *}$ Statistically significant $p<0.05$ via one-way ANOVA with a Dunnett's multiple comparison test $(\mathrm{n}=4)$.

Since many of the critical properties underlying the good performance of CA-PPZ/6MHAPPZ:pDNA nanocarriers were based on their capacity to provide transfection in 3D environments and enhance endosomal escape, we speculated that such advantages would translate to RNA delivery. Therefore, for a therapeutic transgene, we chose a small-interfering RNA to silence DYRK1A (siDYR), a gene implicated in the stability of EGFR presentation and in tumor renewal ${ }^{[10 a, 22]}$. The polymer/siRNA complex formulations were made using the same composition ratio (N/C/P 8:4:1) used for the polymer/pDNA complexes above. Primary characterization showed that substitution of pDNA by siRNA did not induce substantial modifications in nanocarrier characteristics (Figure S11). The DYRK1A silencing efficiency was measured by qRT-PCR of DYRK1A mRNA in U87MG 2D cell cultures (Figure 7a). CA-PPZ/6MHA-PPZ:siDYR complexes were able to silence DYRK1A expression by $70 \%$, while the same prototype carrying a scrambled siRNA sequence (siCtrl) did not 
change DYRK1A expression compared to non-treated cells. In addition, CA-PPZ/6MHAPPZ:siDYR efficacy was higher than the positive control PEI:siDYR complexes, whereas CAPPZ:siDYR complexes did not show target inhibition. As an effect of this DYRK1A inhibition, the renewal capabilities of U87MG were diminished by the treatment with CA-PPZ/6MHA-PPZ:siDYR complexes, as observed in clonogenic assays evaluating the formation of secondary spheroids (Figure 7b). This effect on the ability to form secondary spheroids was also clear in microscopy images (Figure 7c, d, e) and suggested that this gene therapy construct was capable of abrogating the tumor initiating cell capabilities of U87MG cells.

Based on the functional experiments in vitro, the CA-PPZ/6MHA-PPZ complexes showed efficacy in reducing significantly the renewal capability of the GBM cells. We therefore selected this optimized formulation for further proof-of-concepts in vivo. Complexes of CA-PPZ/6MHA-PPZ were used to deliver siDYR $(4 \mu \mathrm{g})$ by intratumoral injection in combination with an intraperitoneal injection of TMZ ( $5 \mathrm{mg} \mathrm{kg}^{-1}$ ) once per day for 4 consecutive days, in the same U87MG-xenografted mouse model (Figure 7f, red arrows). The control group was treated the same TMZ treatment and nanocarrier but with a scrambled siRNA (siCtrl) as payload. In Figure 7g, tumor growth was significantly delayed by CA-PPZ/6MHA-PPZ:siDYR complexes from day 10 to day 14 as compared to the CA-PPZ/6MHA-PPZ:siCtrl group. In fact, a statistical comparison of all the data from day 7 to 17 revealed a strong difference between the growth of tumors treated with siDYR complexes and those treated with siCtrl (i.e. growth of $4.7 \pm 1.4$ fold for siDYR vs. $8.7 \pm 2.9$ fold for siCtrl; $p=0.014$, Student's t-test). This reduction in tumor growth rate can be better appreciated by comparing the size of the treated tumors and those of the controls on the same day (Figure 7g); tumors treated with CAPPZ/6MHA-PPZ:siDYR were around 50\% smaller than those of the control. These data underscored the potential therapeutic superiority when combining the first-line treatment TMZ with CAPPZ/6MHA-PPZ:siDYR complexes in relation to TMZ alone. 
a)

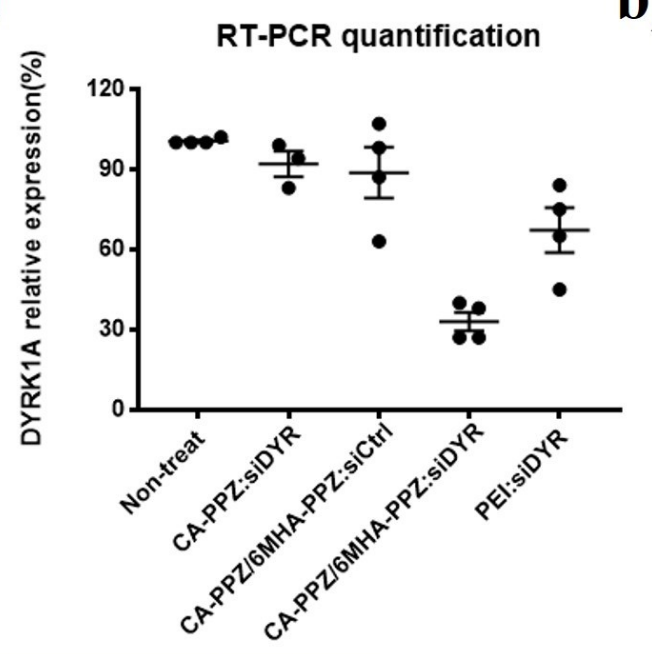

b)

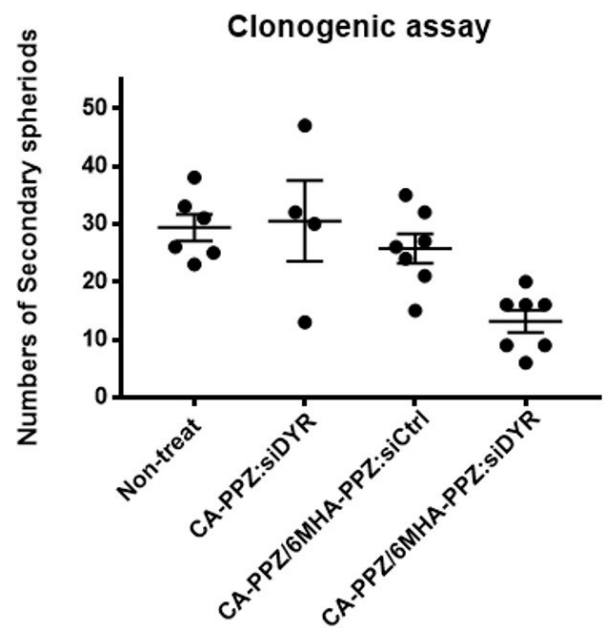

c)

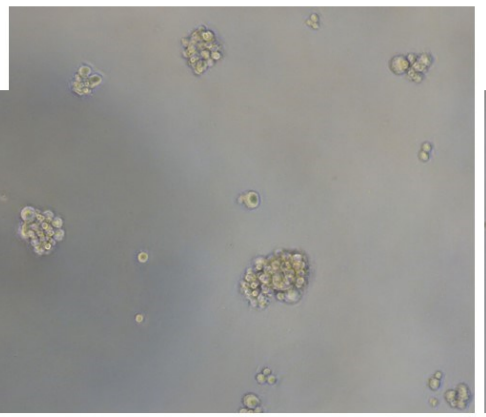

Non-treatment

d)

e)

f)

- CA-PPZ/6MHA-PPZ:siCtrl

曰 CA-PPZ/6MHA-PPZ:siDYR

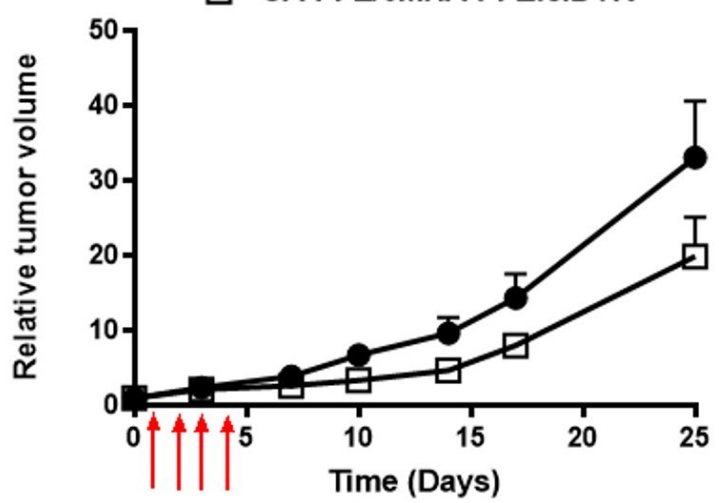

CA-PPZ/6MHA-PPZ:siDYR

CA-PPZ/6MHA-PPZ:siCtrl

g)

- CA-PPZ/6MHA-PPZ:siCtrl

四 CA-PPZ/6MHA-PPZ:siDYR

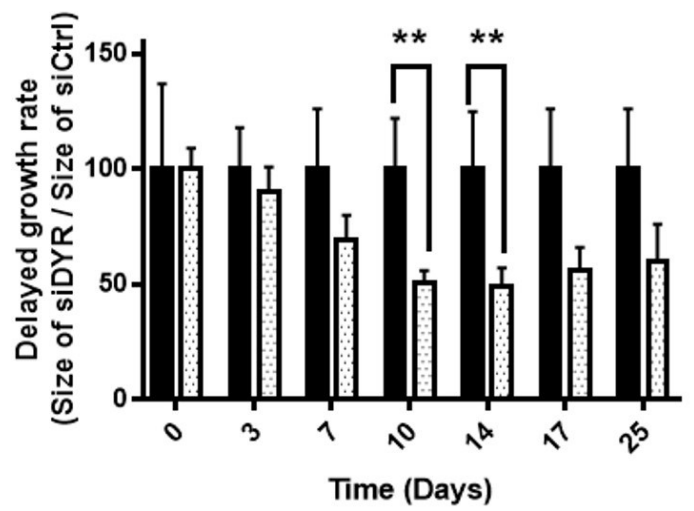

Figure 7. In vitro and in vivo silencing efficacy of polymer:siDYR complexes. a) DYRK1A silencing effect of different polymer:siRNA complexes in a 2D U87MG cell model measured by qRT-PCR . b) Clonogenic ability (formation of secondary spheroids) of U87MG cells after treatment with different polymer:siRNA complexes. Micrographs in $(\mathbf{c}, \mathbf{d}, \mathbf{e})$ show images of secondary spheroids after treatment with CA-PPZ/6MHA-PPZ complexing either a therapeutic (siDYR) or a scrambled siRNA (siCtrl). f) Relative tumor volume in a U87MG xenograft tumor model treated with CAPPZ/6MHA-PPZ:siCtrl or CA-PPZ/6MHA-PPZ:siDYR (4 $\mu \mathrm{g}$ per tumor) and co-therapy with an 
intraperitoneal injection of TMZ $5 \mathrm{mg} \mathrm{kg}^{-1}$. This treatment was repeated for 4 consecutive days (red arrows). g) Delayed tumor growth rate (size of siDYR treated tumors divided by the size of siCtrl treated tumors) at different time points in the same in vivo experiment $(\mathrm{n}=4) . * * P<0.05$, analyzed by unpaired Student's t-test.

\section{Discussion}

Poly(phosphazene)s have been considered as promising materials for gene delivery since they combine biodegradability with high flexibility in terms of chemical diversity. However, their application for gene delivery has remained relatively unexplored because the main synthetic route for polymer modification involves nucleophilic substitution of the precursor poly(dichlorophosphazene) and is problematic for most derivatives of interest. The reason is that functional groups (i.e. amines, hydroxides, carboxylic acids, etc.), often present in biomedical polymers, can react to crosslink the precursor poly(dichlorophosphazene)s. Recently, PPZs have been modified with allylamine and propargylamine as side groups that could be used as click handles in thiol-ene or thiol-yne additions $[6 b, 23]$. Inspired by those studies, we introduced vinyl groups in our systems through the formation of a secondary polymer precursor, allylamino-poly(phosphazene) (AAPPZ), thus enabling further modification with functional groups of interest in a simple step using a thiol-ene addition reaction. This method of generating of "clickable poly(phosphazene)s for gene delivery applications" has the important advantages of requiring no protection or deprotection reactions, and enables facile and rapid preparation of multiple new derivatives with primary amines or carboxylic acid pendent groups. The streamlined synthetic process thus enabled structure/performance relationships to be derived rapidly in terms of in vitro transgene expression efficiency.

An important finding from the initial screening experiments was the identification of 6MHAPPZ as a material with gene delivery enhancing properties. This polymer was designed to perform three different functions in the delivery system: (i) improve interpolymer complexation during the formation of the mixed polyelectrolyte, (ii) promote the release of polynucleotides from 
endolysosomal compartments, and (iii) reduce polycation-caused toxicity. The capacity of anionic PPZs to enhance interpolymer complexation was confirmed by comparing CA-PPZ:pDNA (8:1 N/P ratio) and CA-PPZ/6MHA-PPZ:pDNA (8:4:1 N/C/P ratio) complexes via dynamic light scattering and TEM imaging. Dynamic light scattering showed an almost one order of magnitude increase in derived count rate when 6MHA-PPZ was added (Figure S4c). TEM images showed that CAPPZ/6MHA-PPZ:pDNA complexes had more compact morphologies than CA-PPZ:pDNA complexes (Figure 2c, d). Many standard polymers used for gene delivery are typically used in excess with the polynucleotide. For example, while it is necessary only to use an N/P ratio of 3 when aiming to form colloidally stable complexes of pDNA with PEI, in practice much larger excesses of PEI are used for improving in vitro transfection efficiency, thus resulting in weakly bound or unbound PEI ${ }^{[24]}$. However, there are concerns regarding the fate and side effects of these unbound polymers when these systems are administered in vivo. In comparison, the dense network of the CA-PPZ/6MHAPPZ:pDNA complexes as shown in the TEM images suggested that these formulations were more closely associated and thus more likely to be delivered as intact complexes to the target cells.

A second observation regarding the function of $6 \mathrm{MHA}-\mathrm{PPZ}$ in the nanocomplexes was its capacity to increase the efficacy/toxicity ratio over one order of magnitude, reaching levels similar to the laboratory transfection standard PEI:pDNA in 2D models of U87MG cells (Figure 2e), and showing superior efficacies in a clinically relevant glioblastoma primary cell line (GBM1, Figure S6). Indeed, we observed that introduction of all polyanionic-PPZs in the complexes reduced the overall cellular toxicity. We infer that this reduction was partly driven by charge neutralization, an effect already reported with other polyanions ${ }^{[25]}$. However, the other polyanionic PPZs failed to enhance the efficacy of gene delivery compared to 6MHA-PPZ (Figure 2e). Microscopy showed that the presence of 6MHA-PPZ in the complexes resulted in lower co-localization with the endosomal compartments (Figure 3b), suggesting that this improvement in transfection could be related to higher capacity of these formulations to exit the endosomal vesicles. Since this improvement in transfection was specific for 6MHA-PPZ, we think it resulted from the change in ionization degree of 6MHA-PPZ across the early endosomal $\mathrm{pH}$ range, which was the largest among all the polyanions 
we tested (Table S1, Figure 1c). This change in ionization was most probably linked to the capacity of 6MHA-PPZ to rupture lipid membranes in the acid environment of the endosomes as confirmed in hemolysis tests (Figure S7). Complexes containing 6MHA-PPZ were the only ones that exhibited negligible hemolytic effects at neutral $\mathrm{pH}$, but a hemolytic effect comparable to poly-L-lysine (PLL) at acidic $\mathrm{pH}$. It is also of note that 6MHA-PPZ was capable of improving cell transfection when combined with polycations known to possess low endosomal escape properties, such as PLL, although the combination with CA-PPZ was the most efficient in the different cell-lines (Figure S6), likely attributed to the optimization above (Figure S5a,b).

Traditional two-dimensional cell monolayer models have been widely used for optimization of formulations, but also have been questioned for their ability to represent physiologically relevant situations. Three-dimensional cultures are better able to provide tissue-like architectures (e.g. extracellular matrix, cell interactions) ${ }^{[26]}$, fundamental tumor microenvironment traits such as nutrient and oxygen gradients ${ }^{[27]}$, and also gene expression profiles similar to those of clinical tumors ${ }^{[28]}$. To date, very few studies have investigated nucleic acid delivery in tumor spheroids despite the fact that they are highly suited as preliminary assays before in vivo efficacy studies and that they can provide critical information regarding tumor penetration, which is a critical barrier in cancer drug delivery ${ }^{[29]}$. For our investigations, we used FLSM to obtain tomographic fluorescence images of GFP transfection expression without damaging the initial spheroid structure. As apparent in Figure 5, CAPPZ/6MHA-PPZ:pDNA complexes exhibited higher transfection in GBM spheroids as compared to CA-PPZ:pDNA and the reference PEI:pDNA complexes; this difference was particularly marked at low pDNA doses. This improved efficacy was also accompanied by reduced toxicity. Overall, these results showed high transfection efficacy and tumor penetration capacity for the CA-PPZ/6MHAPPZ:pDNA formulation, which we attribute to their partially neutralized cationic charges at physiological $\mathrm{pH}$ and lower tendency to bind to the extracellular matrix in tumor spheroids. The results were also in agreement with those from subsequent in vivo gene delivery experiments, although direct correlation between these assays is problematic due to inhomogeneous transfection levels between injection and distal sites (Figure 6). 
GBM initiating cells are considered to be responsible for tumor recurrence ${ }^{[30]}$, and therefore, their suppression is essential for any medicine intended to provide mid- or long-term survival benefits. In the past, we have shown that controlled release implants can deliver glioblastoma initiating cell suppressing molecules and provide a therapeutic benefit in advanced in vivo models ${ }^{[31]}$. Because the critical properties behind the performance of this formulation are barriers common to both pDNA and RNA delivery (i.e. endosomal escape, transfection in 3D environments), following the pilot in vivo study with pDNA, we applied the optimized polymer prototype to deliver a siRNA sequence with known activity against glioblastoma initiating cells (siDYR) ${ }^{[10 a]}$. As in the case of the DNA complexes, the use of 6MHA-PPZ mixed in with the CA-PPZ complexes provided sufficient ionic attraction for complexation, and as a result the physicochemical properties of CA-PPZ/6MHAPPZ:siRNA were similar to CA-PPZ/6MHA-PPZ:pDNA (Figure 11). We also confirmed that protein silencing observed for cells treated with CA-PPZ/6MHA-PPZ:siDYR resulted in a reduction in the clonogenic index in a secondary spheroid formation assay, indicating the abrogation of the "initiating cell" phenotype of the target cells, an effect that could have major clinical relevance in combination therapies. Since we expect gene therapy to provide initial clinical benefits in neuro-oncology as adjuvants in combination with antitumoral drugs, the CA-PPZ/6MHA-PPZ:siDYR formulation was evaluated in U87MG xenografted mice, in a therapeutic scheme including co-delivery of the first-line treatment TMZ. The experiment showed a significant delay in tumor progression in the treatment arm receiving TMZ and CA-PPZ/6MHA-PPZ:siDYR vs. the study arm receiving TMZ and CAPPZ/6MHA-PPZ complexed with a scrambled siRNA sequence (Figure 7f, g). Therefore, our results indicate an additional therapeutic benefit for the combination of CA-PPZ/6MHA-PPZ:siDYR gene therapy with the standard pharmacological GBM treatment, as compared to this standard treatment alone. Further studies will be needed to elucidate the possible clinical impact of this combined therapy in more advanced models and performing additional phenotypic analysis in the tumors.

\section{Conclusion}

A synthetic strategy for the simple preparation of a small library of poly(phosphazene)s of 
interest for gene delivery was developed based on the precursor polymer with "clickable" sites, and further derivation by thiol-ene additions. This approach allowed us to test a variety of compounds and draw structure/function relationships for gene delivery. Based on the screenings, a primary aminecontaining polymer (CA-PPZ) and an anionic poly(phosphazene) analogue (6MHA-PPZ) were selected for plasmid DNA gene delivery. These binary polymer/pDNA complexes displayed significantly higher transfection efficiencies than the parent polycation-only system in 2D monolayers, 3D spheroids of U87MG cells and in a subcutaneous xenograft model, due to improved tumor transport and intracellular trafficking characteristics. This optimized prototype also showed indications of superior performance compared to laboratory standard PEI complexes, particularly in 3D tumor models. When used for RNAi therapy, CA-PPZ/6MHA-PPZ complexes efficiently delivered siRNA against DYRK1A in U87MG cells, inducing a significant reduction of their selfrenewal capability in vitro and a significant anti-tumor effect in an in vivo glioblastoma model. Overall, the data established a new versatile, biodegradable polymeric gene delivery based on poly(phosphazene)s with high capacity for gene-transfer efficacy in vitro and upon in-situ treatment in vivo.

\section{Acknowledgements}

We thank the European Commission for an Erasmus Mundus grant to WHH (NanoFar Joint Doctorate Program). This work was funded by the Engineering and Physical Sciences Research Council (Grants EP/H005625/1, EP/N03371X/1) and the Royal Society (Wolfson Research Merit Award WM150086) to CA, Ministerio de Economía y Competitividad (MINECO-RETOS, Grant SAF2015-65175-R to PSG and SAF2014-58189-R to MGF, FEDER Funds) and Fundación BBVA 2014-PO0110 to MGF. The pEGFPLuc plasmid DNA was a kind gift from Prof. A. Vidal and Dr. C. Carneiro (University of Santiago de Compostela). The primary GBM cell-line GBM1 was a kind donation from Dr. MD. Bernard Rogister (University of Liege). We acknowledge Pamela Collier, Christine Grainger-Boultby, Paul Cooling, Dr. Manuel Martin-Pastor and Dr. Jose Manuel Ageitos 
for expert technical support. We also appreciate help from Dr. Esther Hernández and Paloma Fernández in molecular biological assays and in vivo studies.

\section{Data access statement and supplementary information}

All raw data created during this research are openly available from the corresponding authors, Cameron.alexander@nottingham.ac.uk (CA) and marcos.garcia@usc.es (MGF), and at the University of Nottingham Research Data Management Repository (https://rdmc.nottingham.ac.uk/) and MINERVA (USC Institutional Repository, https://minerva.usc.es). Further data supporting these studies is provided as a separate document in supplementary information and three videos. The videos show tomographic images of 3D spheroids treated with the complexes loaded with a pDNA encoding GFP. Blue channel is for DAPI staining and green for GFP. Videos correspond to spheroids treated with: (i) buffer (“Control tomography”), (ii) CA-PPZ complexes (“CA-PPZ tomography”) and (iii) CA-PPZ/6MHA-PPZ complexes (“CA-PPZ_6MHA-PPZ tomography”).

\section{Conflict-of-Interest Disclosure}

The authors declare no conflicts of interest. 


\section{References}

[1] a) A. N. Zelikin, C. Ehrhardt, A. M. Healy, Nat Chem 2016, 8, 997; b) R. Kanasty, J. R. Dorkin, A. Vegas, D. Anderson, Nat Mater 2013, 12, 967; c) H. Yin, R. L. Kanasty, A. A. Eltoukhy, A. J. Vegas, J. R. Dorkin, D. G. Anderson, Nature Reviews Genetics 2014, 15, 541.

[2] R. Titze-de-Almeida, C. David, S. S. Titze-de-Almeida, Pharmaceutical Research 2017, 34, 1339.

[3] a) C. Scholz, E. Wagner, Journal of Controlled Release 2012; b) A. Krivitsky, D. Polyak, A. Scomparin, S. Eliyahu, A. Ori, S. Avkin-Nachum, V. Krivitsky, R. Satchi-Fainaro, Biomacromolecules 2016, 17, 2787; c) S. M. Moghimi, E. Wagner, Molecular Therapy 2017, 25, 1461; d) U. Lachelt, E. Wagner, Chemical Reviews 2015, 115, 11043.

[4] H. R. Allcock, Science 1976, 193, 1214.

[5] a) A. P. Martinez, B. Qamar, T. R. Fuerst, S. Muro, A. K. Andrianov, Biomacromolecules 2017, 18, 2000; b) Y. Peng, X. M. Zhu, L. Y. Qiu, Biomaterials 2016, 106, 1; c) M. H. Gao, X. M. Zhu, L. P. Wu, L. Y. Qiu, Biomacromolecules 2016, 17, 2199; d) C. Y. Ma, X. Zhang, C. G. Du, B. J. Zhao, C. H. He, C. Li, R. Z. Qiao, Bioconjugate Chemistry 2016, 27, 1005; e) L. J. Meng, C. Q. Xu, T. H. Liu, H. Li, Q. H. Lu, J. G. Long, Polymer Chemistry 2015, 6, 3155; f) I. Teasdale, O. Bruggemann, Polymers 2013, 5, 161; g) P. C. Zhang, Z. W. Zhang, Y. X. Yang, Y. P. Li, International Journal of Pharmaceutics 2010, 392, 241.

[6] a) X. Huang, X. J. Huang, A. G. Yu, C. Wang, Z. W. Dai, Z. K. Xu, Macromolecular Chemistry and Physics 2011, 212, 272; b) Y. C. Qian, X. J. Huang, C. Chen, N. Ren, X. Huang, Z. K. Xu, Journal of Polymer Science Part A: Polymer Chemistry 2012, 50, 5170.

[7] a) A. K. Varkouhi, M. Scholte, G. Storm, H. J. Haisma, Journal of Controlled Release 2011, 151, 220; b) T. Endoh, T. Ohtsuki, Advanced drug delivery reviews 2009, 61, 704; c) H. C. Kang, Y. H. Bae, Advanced Functional Materials 2007, 17, 1263.

[8] E. G. Van Meir, C. G. Hadjipanayis, A. D. Norden, H. K. Shu, P. Y. Wen, J. J. Olson, CA Cancer J Clin 2010, 60, 166.

[9] a) M. Westphal, D. C. Hilt, E. Bortey, P. Delavault, R. Olivares, P. C. Warnke, I. R. Whittle, J. Jääskeläinen, Z. Ram, Neuro-oncology 2003, 5, 79; b) R. Stupp, W. P. Mason, M. J. Van Den Bent, M. Weller, B. Fisher, M. J. Taphoorn, K. Belanger, A. A. Brandes, C. Marosi, U. Bogdahn, New England Journal of Medicine 2005, 352, 987.

[10] a) N. Pozo, C. Zahonero, P. Fernández, J. M. Liñares, A. Ayuso, M. Hagiwara, A. Pérez, J. R. Ricoy, A. Hernández-Laín, J. M. Sepúlveda, The Journal of clinical investigation 2013, 123, 2475; b) S. R. Ferron, N. Pozo, A. Laguna, S. Aranda, E. Porlan, M. Moreno, C. Fillat, S. de la Luna, P. Sánchez, M. L. Arbonés, Cell stem cell 2010, 7, 367.

[11] a) F. J. Attenello, D. Mukherjee, G. Datoo, M. J. McGirt, E. Bohan, J. D. Weingart, A. Olivi, 
A. Quinones-Hinojosa, H. Brem, Annals of surgical oncology 2008, 15, 2887; b) M. J.

McGirt, K. D. Than, J. D. Weingart, K. L. Chaichana, F. J. Attenello, A. Olivi, J. Laterra, L.

R. Kleinberg, S. A. Grossman, H. Brem, Journal of neurosurgery 2009, 110, 583.

[12] Y. S. Sohn, Y. H. Cho, H. Baek, O.-S. Jung, Macromolecules 1995, 28, 7566.

[13] A. K. Andrianov, J. Chen, M. P. LeGolvan, Macromolecules 2004, 37, 414.

[14] Y.-C. Qian, P.-C. Chen, G.-J. He, X.-J. Huang, Z.-K. Xu, Molecules 2014, 19, 9850.

[15] D. P. Ivanov, T. L. Parker, D. A. Walker, C. Alexander, M. B. Ashford, P. R. Gellert, M. C. Garnett, PLoS One 2014, 9, e103817.

[16] C. Zahonero, P. Aguilera, C. Ramírez-Castillejo, M. Pajares, M. V. Bolós, D. Cantero, A. Perez-Nuñez, A. Hernández-Laín, P. Sánchez-Gómez, J. M. Sepúlveda, Molecular cancer therapeutics 2015, 14, 1548.

[17] K. T. Love, K. P. Mahon, C. G. Levins, K. A. Whitehead, W. Querbes, J. R. Dorkin, J. Qin, W. Cantley, L. L. Qin, T. Racie, Proceedings of the National Academy of Sciences 2009, 107, 1864.

[18] T. F. Martens, K. Remaut, J. Demeester, S. C. De Smedt, K. Braeckmans, Nano Today 2014, 9, 344 .

[19] J. Kroonen, J. Nassen, Y. G. Boulanger, F. Provenzano, V. Capraro, V. Bours, D. Martin, M. Deprez, P. Robe, B. Rogister, International Journal of Cancer 2011, 129, 574.

[20] P. J. Kelly, Surgical neurology international 2010, 1, 96.

[21] D. P. Ivanov, A. M. Grabowska, Scientific Reports 2017, 7, 41160.

[22] a) R. Abbassi, T. G. Johns, M. Kassiou, L. Munoz, Pharmacology \& therapeutics 2015, 151, 87; b) P. Fernandez-Martinez, C. Zahonero, P. Sanchez-Gomez, Molecular \& cellular oncology 2015, 2, e970048.

[23] N. Ren, X. J. Huang, X. Huang, Y. C. Qian, C. Wang, Z. K. Xu, Journal of Polymer Science Part A: Polymer Chemistry 2012, 50, 3149.

[24] a) S. Boeckle, K. von Gersdorff, S. van der Piepen, C. Culmsee, E. Wagner, M. Ogris, The journal of gene medicine 2004, 6, 1102; b) Y. Yue, F. Jin, R. Deng, J. Cai, Y. Chen, M. C. Lin, H.-F. Kung, C. Wu, Journal of Controlled Release 2011, 155, 67.

[25] a) L. Han, C. Tang, C. Yin, Biomaterials 2013, 34, 5317; b) T. Kurosaki, T. Kitahara, S. Fumoto, K. Nishida, J. Nakamura, T. Niidome, Y. Kodama, H. Nakagawa, H. To, H. Sasaki, Biomaterials 2009, 30, 2846; c) V. Trubetskoy, S. Wong, V. Subbotin, V. Budker, A. Loomis, J. Hagstrom, J. Wolff, Gene Therapy 2003, 10, 261.

[26] S. A. Enam, E. Klaus, Neurosurgery 1998, 42, 599.

[27] R. M. Sutherland, B. Sordat, J. Bamat, H. Gabbert, B. Bourrat, W. Mueller-Klieser, Cancer Research 1986, 46, 5320.

[28] P. D. W. Hamer, A. Van Tilborg, P. Eijk, P. Sminia, D. Troost, C. Van Noorden, B. Ylstra, S. Leenstra, Oncogene 2008, 27, 2091.

[29] a) J. Zhou, T. R. Patel, R. W. Sirianni, G. Strohbehn, M.-Q. Zheng, N. Duong, T. Schafbauer, A. J. Huttner, Y. Huang, R. E. Carson, Proceedings of the National Academy of Sciences 2013, 110, 11751; b) E. Song, A. Gaudin, A. R. King, Y.-E. Seo, H.-W. Suh, Y. Deng, J. Cui, 

G. T. Tietjen, A. Huttner, W. M. Saltzman, Nature communications 2017, 8, 15322.

[30] C. Garcia-Mazas, N. Csaba, M. Garcia-Fuentes, International Journal of Pharmaceutics 2017, 523, 490.

[31] a) E. Reguera-Nuñez, C. Roca, E. Hardy, M. de la Fuente, N. Csaba, M. Garcia-Fuentes, Biomaterials 2014, 35, 2859; b) P. González-Gómez, J. Crecente-Campo, C. Zahonero, M. de la Fuente, A. Hernández-Laín, H. Mira, P. Sánchez-Gómez, M. Garcia-Fuentes, Oncotarget 2015, 6, 10950. 


\section{Supporting Information}

\section{Title: Structure-optimized interpolymer polyphosphazene complexes for effective gene delivery to glioblastoma}

Author(s): Wei-Hsin Hsu, ${ }^{1,2}$ Pilar Sánchez-Gómez, ${ }^{3}$ Esther Gomez-Ibarlucea, ${ }^{1}$ Delyan P.

Ivanov, ${ }^{4}$ Ruman Rahman, ${ }^{4}$ Anna M. Grabowska,${ }^{4}$ Noemi Csaba, ${ }^{1}$ Cameron Alexander ${ }^{2 *}$ and

Marcos Garcia-Fuentes ${ }^{1 *}$

1 - Centre for Research in Molecular Medicine and Chronic Diseases (CIMUS), Universidade de Santiago de Compostela, Spain

2 - Division of Molecular Therapeutics and Formulation, School of Pharmacy, University of Nottingham, Nottingham NG7 2RD, UK

3 - Neurooncology Unit, Instituto de Salud Carlos III (UFIEC), Madrid, Spain

4 - Division of Cancer and Stem Cells, School of Medicine, Queen's Medical Centre, University of Nottingham, Nottingham NG7 2RD, UK

*Corresponding authors:

E-mail: cameron.alexander@nottingham.ac.uk (Cameron Alexander)

E-mail: marcos.garcia@usc.es (Marcos Garcia-Fuentes) 
a)

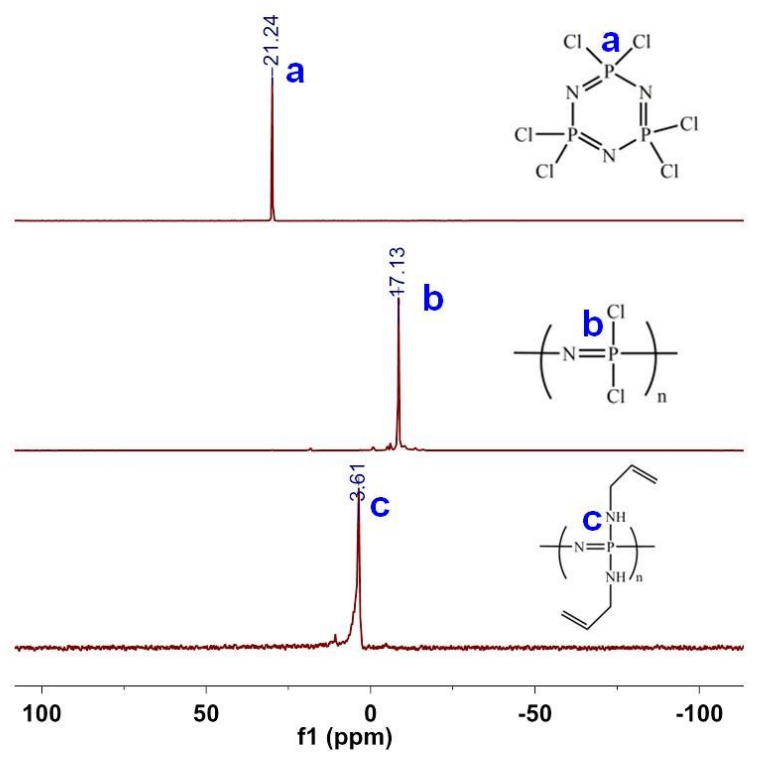

b)

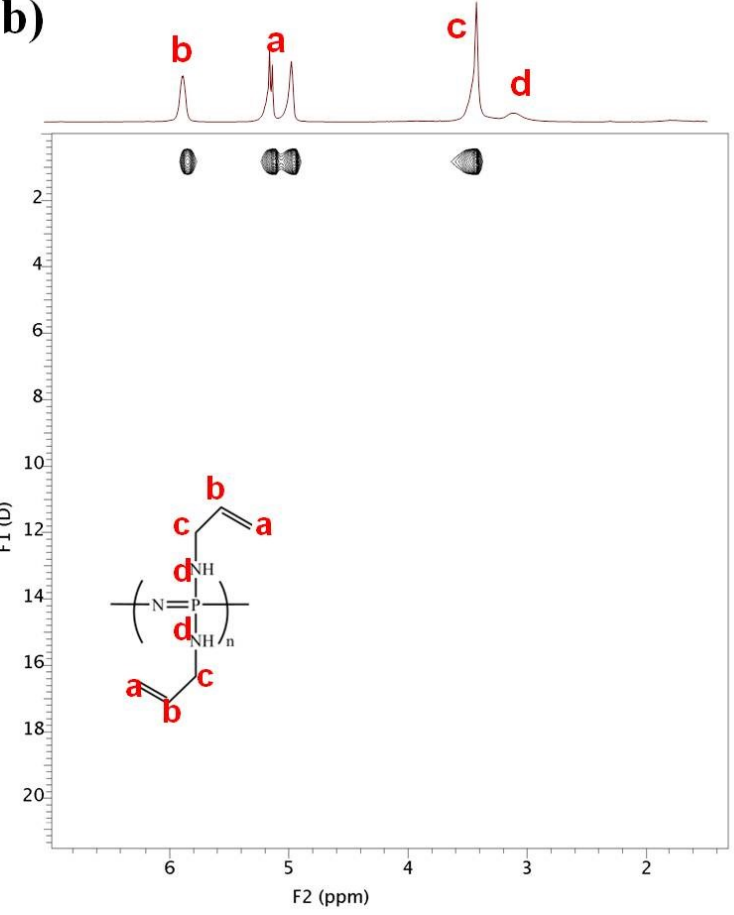

Figure S1 (a) ${ }^{31}$ P-NMR spectra of hexachlorocyclotriphosphazene, poly(dichlorophosphazene) (PDCP), and allylamino-polyphosphazene (AAPPZ). (b) ${ }^{1} \mathrm{H}-\mathrm{NMR}-\mathrm{DOSY}$ of AAPPZ.

a)

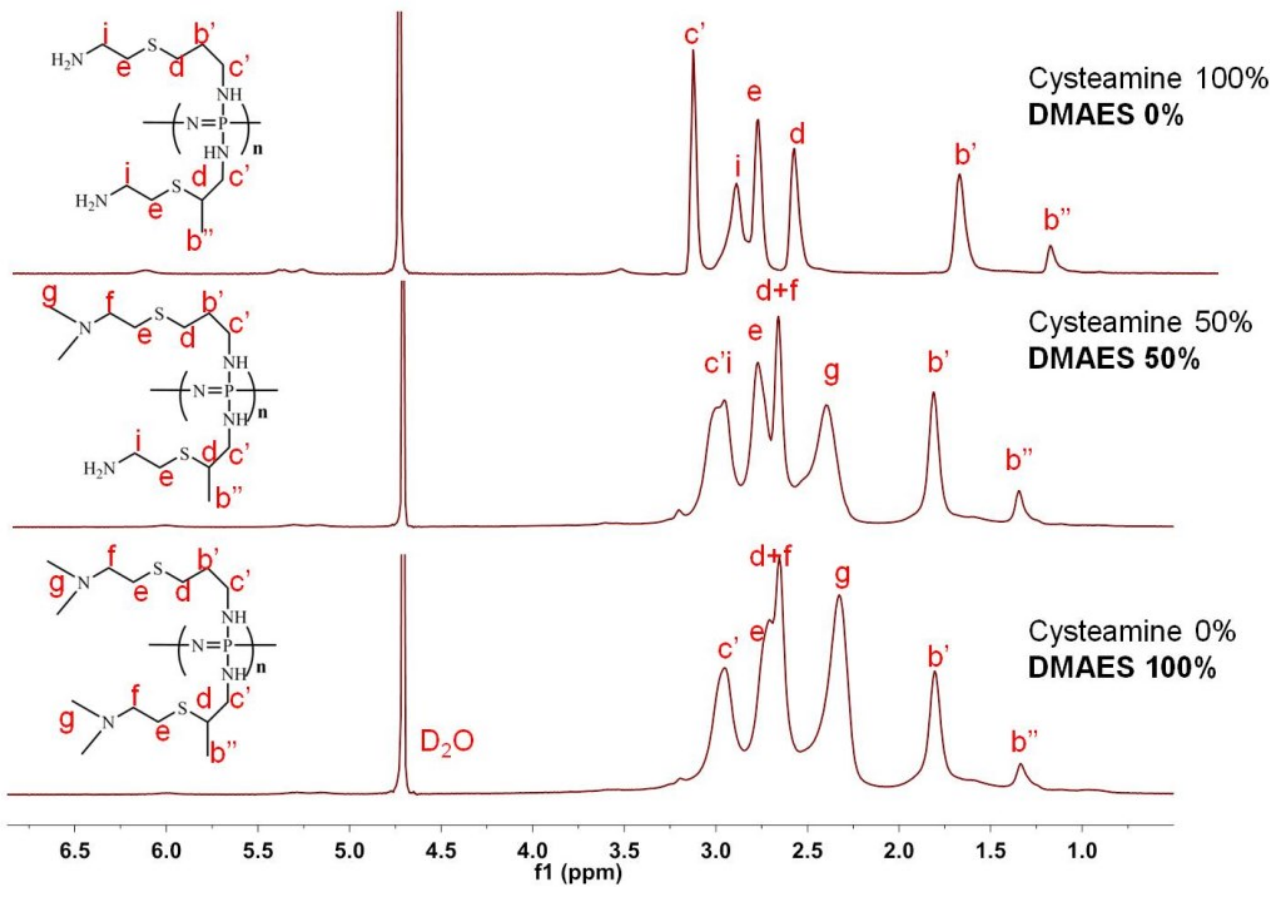




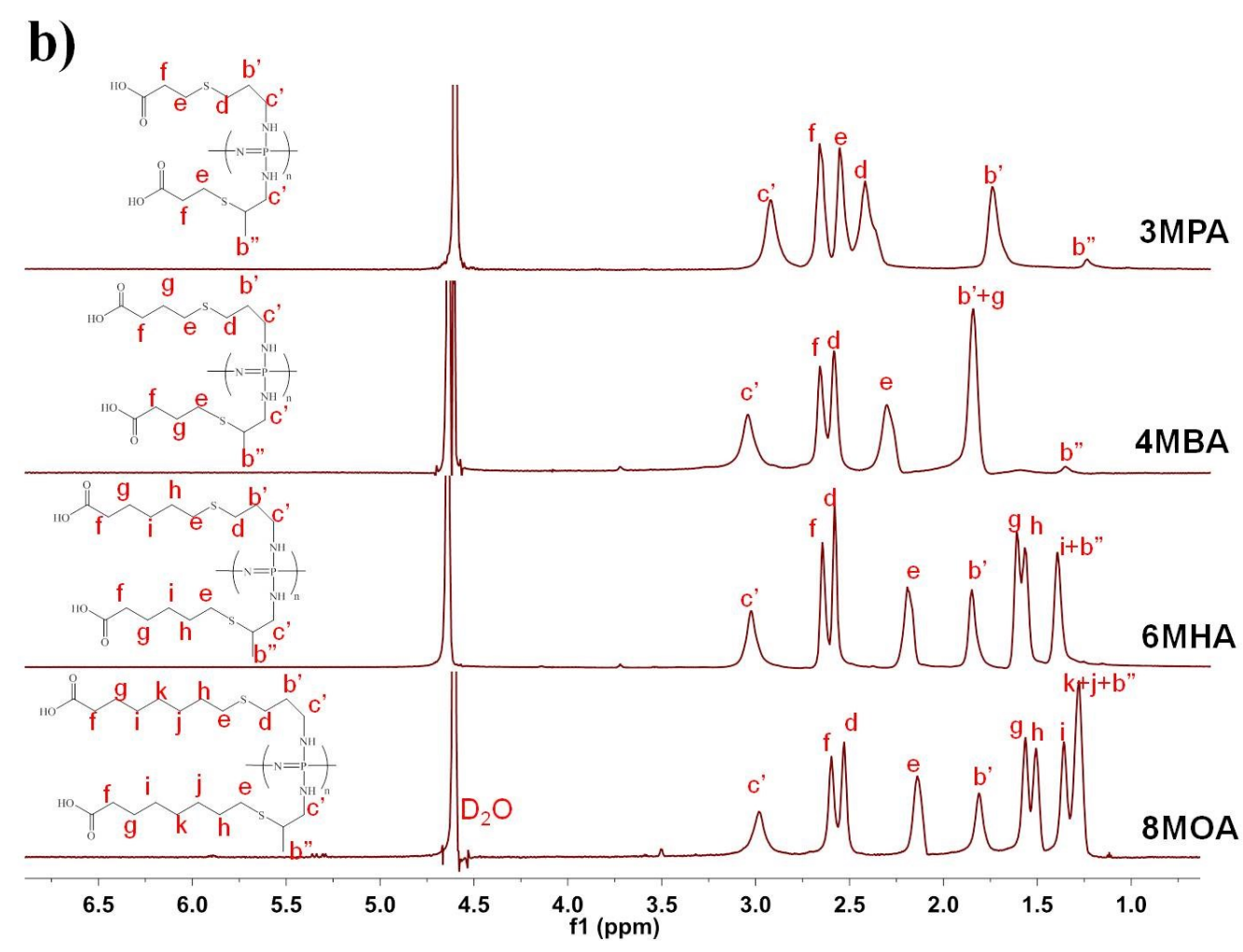

Figure S2. (a) ${ }^{1} \mathrm{H}-\mathrm{NMR}$ of cationic polyphosphazenes functionalized with cysteamine and/or 2-(dimethylamino)ethanethiol (DMAES) at group ratios (Cysteamine: DMAES) of 100:0, 50:50, and 0:100. The polymers were dissolved in $\mathrm{D}_{2} \mathrm{O}$ around $\mathrm{pH} 9-10$. (b) ${ }^{1} \mathrm{H}-\mathrm{NMR}$ of anionic functionalized polyphosphazenes with aliphatic chains of diverse lengths, prepared via thiol-ene addition.

Abbreviations: 3-Mercaptopropanoic acid (3MPA), 4-Mercaptobutanoic acid (4MBA), 6-Mercaptohexanoic acid (6MHA), 8-Mercaptooctanoic acid (8MOA) as side groups linked on the polyphosphazene backbone. 
a)

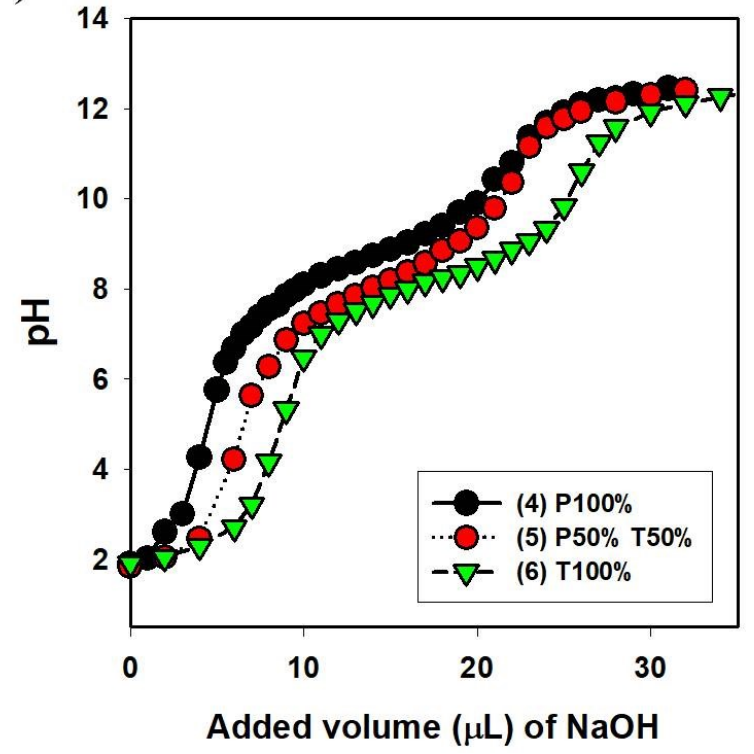

b)

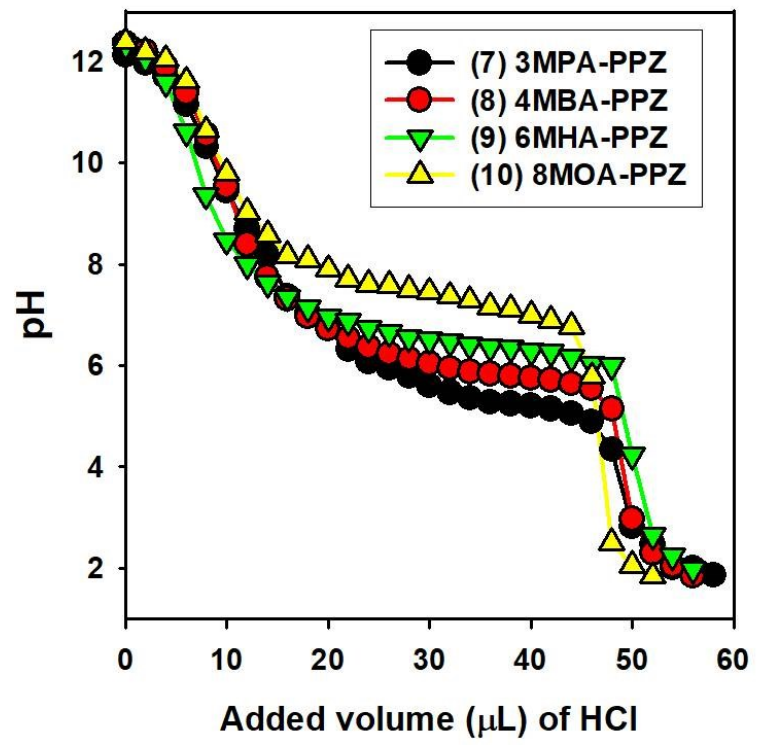

Figure S3. (a) Potentiometric titration curves of cationic polyphosphazenes with: only primary amines attached $(\mathrm{P} 100 \%,(4)$ in the structure scheme), half primary and half tertiary amines attached (P50\%T50\%, (5)), and only tertiary amine attached (T100\%) (6). (b) Potentiometric titration curves of different anionic polyphosphazenes. 
a)

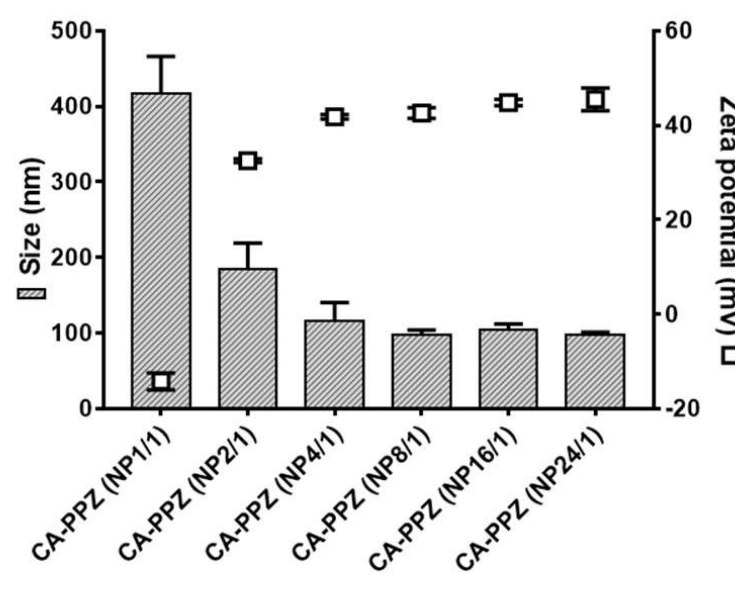

b)

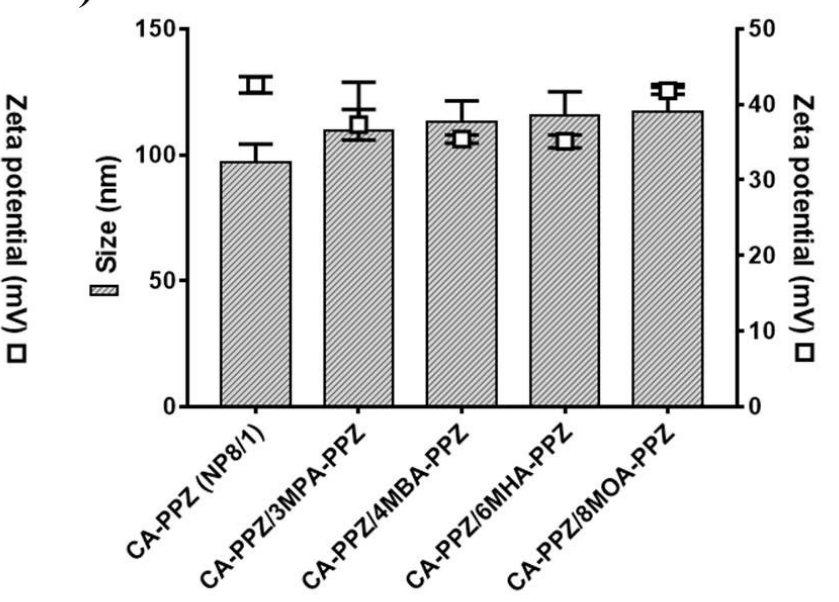

c)

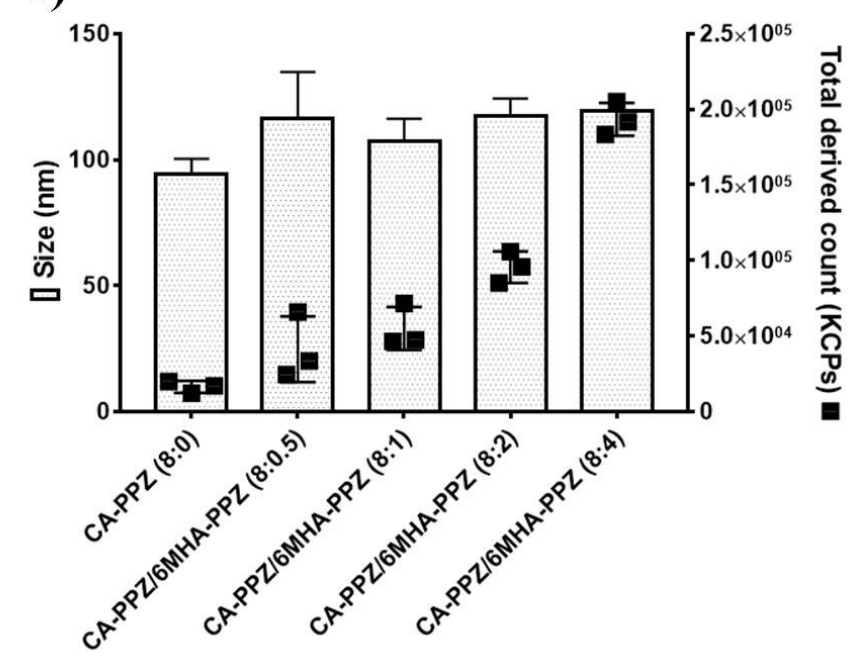

Figure S4. Characterization of ionic PPZ gene complexes. Hydrodynamic size and zeta potential of a) CA-PPZ:pDNA with different N/P ratios, and b) CA-PPZ:pDNA complexes mixed with different polyanions, 3MPA-, 4MBA-, 6MHA-, and 8MOA-PPZ. In b) a ratio of CA-PPZ amines, anionic PPZ carboxylates and pDNA phosphorous (N/C/P) of 8:4:1 was used. c) The particle sizes and total derived counts (KCPs) of CA-PPZ:pDNA complexes (8:1 N/P ratio) mixed with increasing amounts of 6MHA-PPZ (from 8:0 to 8:4 N/C ratio). Data presented by mean $\pm \mathrm{SD}(\mathrm{n}=3)$. 
a)
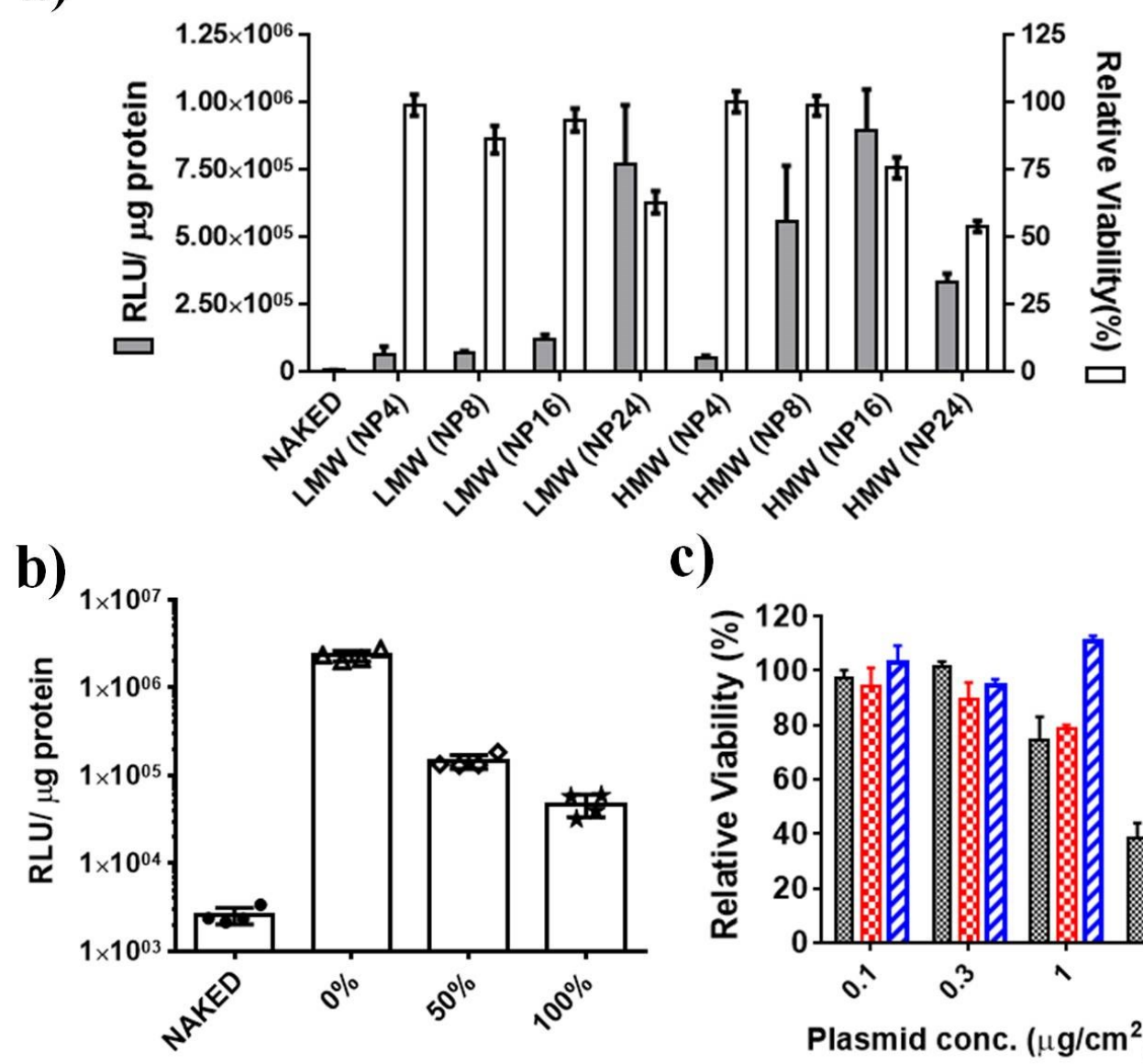

c)

Tertiary amine (\%) in whole polymer

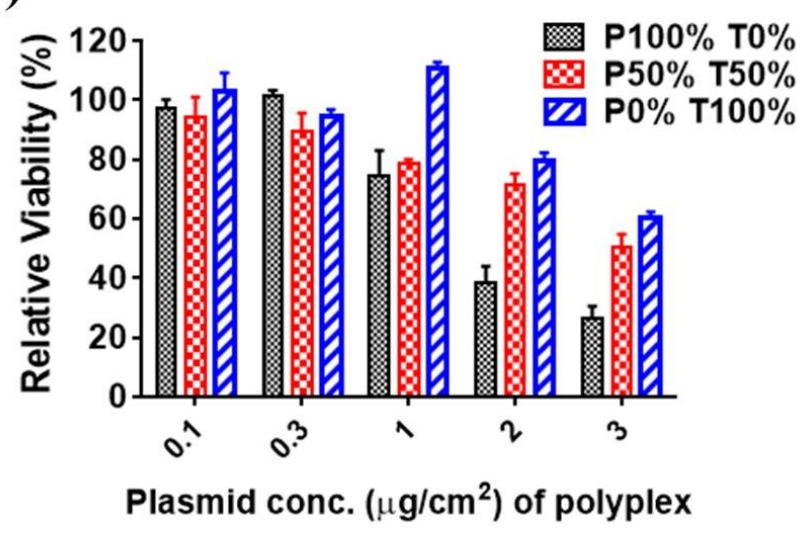

Figure S5. Optimization of polycationic complexes in 2D-culture U87MG cells. (a) Luciferase expression and cell viability of complexes with low molecular weight (LMW 26K Da) and high molecular weight (HMW 36K Da) of CA-PPZ at different N/P ratios with pDNA. Luciferase expression upon transfection with complexes of different polycationic PPZs, at $1 \mu \mathrm{g}$ $\mathrm{cm}^{-2}$ pDNA dose and 8:1 N/P ratios. (c) Cell viability $48 \mathrm{~h}$ after transfection with the same polycationic PPZs. In both b) and c) the percentage labels of the different experimental groups represent the fraction of tertiary amines (DMAES), being the rest primary amines (cysteamine). Data presented by mean $\pm \operatorname{SEM}(n=4)$. 


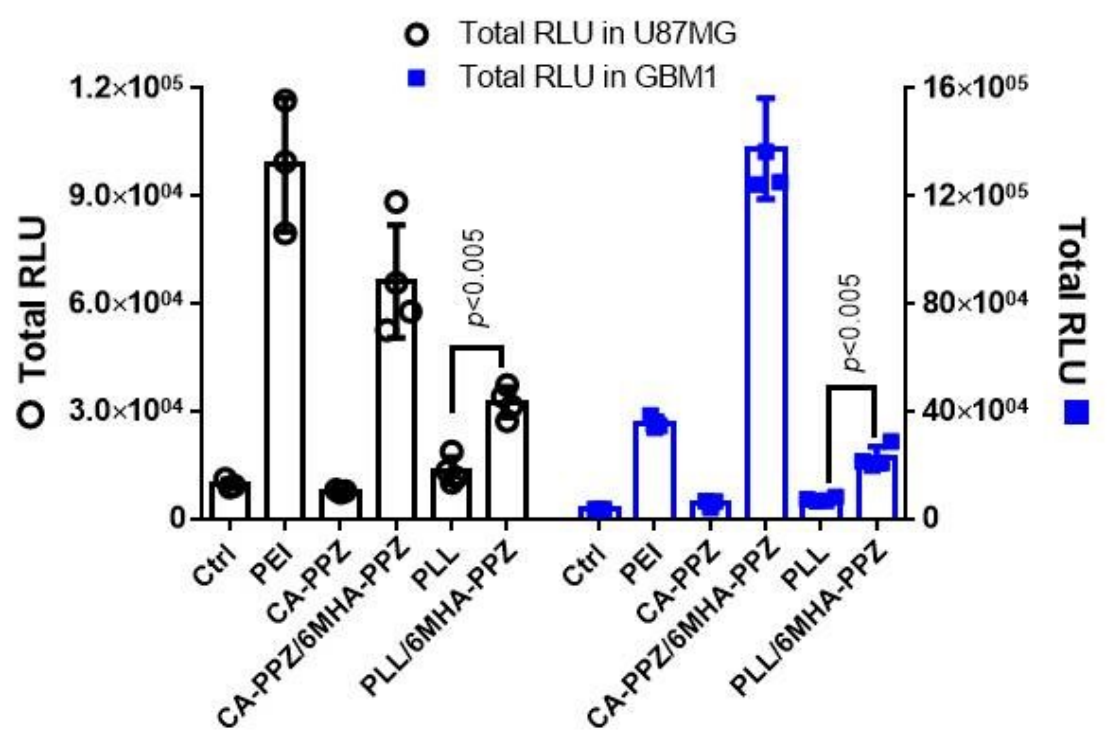

Figure S6. Luciferase expression upon cell transfection with CA-PPZ and poly-L-lysine (PLL) alone (8:1 N/P ratios) and after mixing with 6MHA-PPZ (8:4:1 N/C/P ratio). Data obtained in U87MG and in the primary cell line GBM1. Data presented by mean $\pm \operatorname{SEM}(n \geq 3)$.

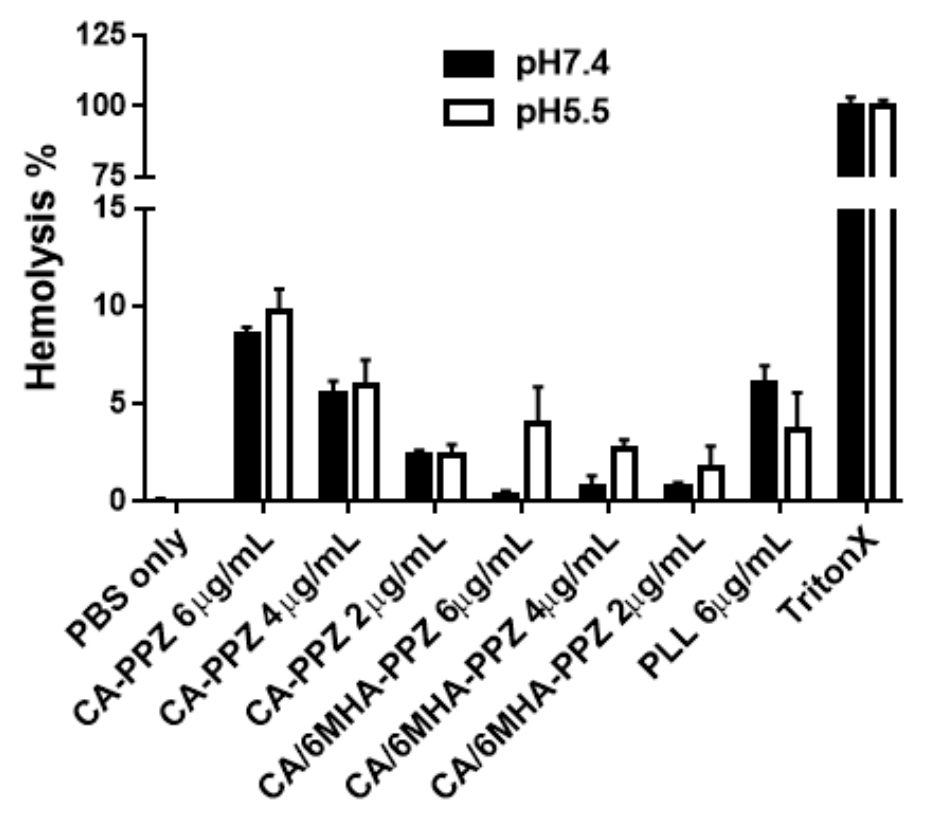

Figure S7. Hemolysis percentages of CA-PPZ complex (CA-PPZ:pDNA, N/P 8:1), CA-PPZ/6MHA-PPZ:pDNA complex (N/C/P 8:4:1) with different doses incubated with murine 
red blood cells for $2 \mathrm{~h}$ for $37{ }^{\circ} \mathrm{C}$. Note: the $100 \%$ hemolysis is defined by the absorption value via treatment of $1 \% \mathrm{v} / \mathrm{v}$ Triton $X-100$. Data presented by mean $\pm \operatorname{SEM}(n=4)$.

a)

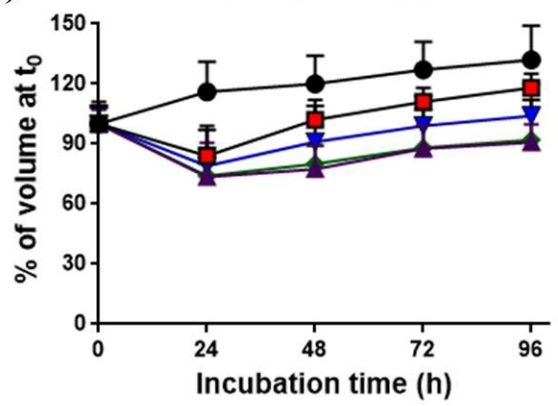

c)

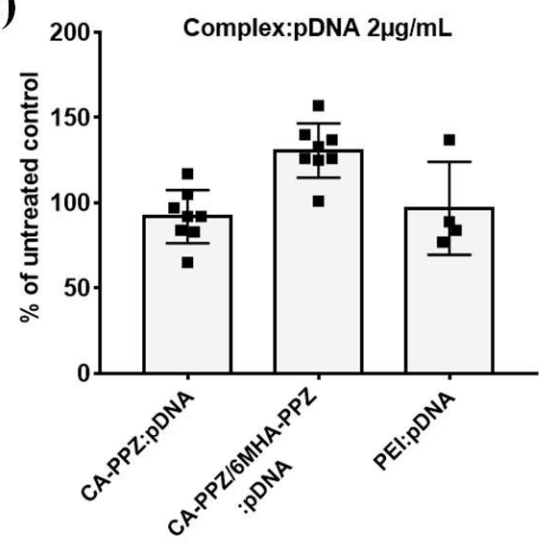

b)

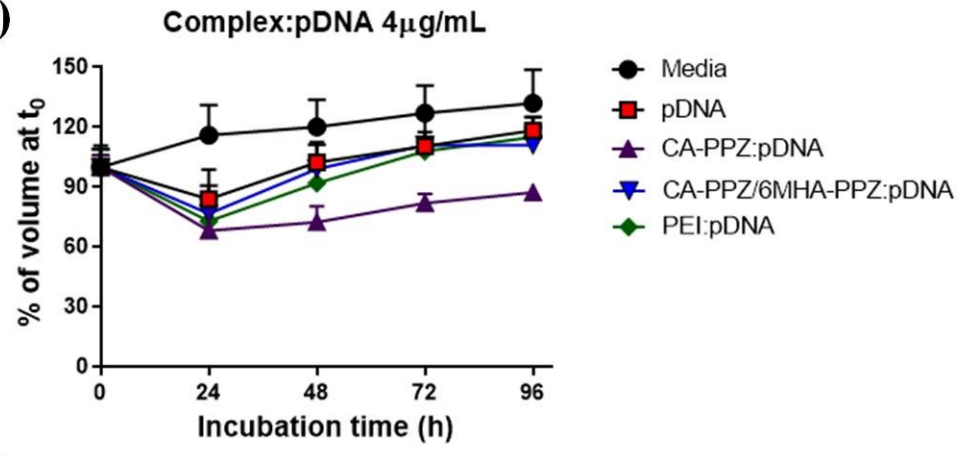

d)

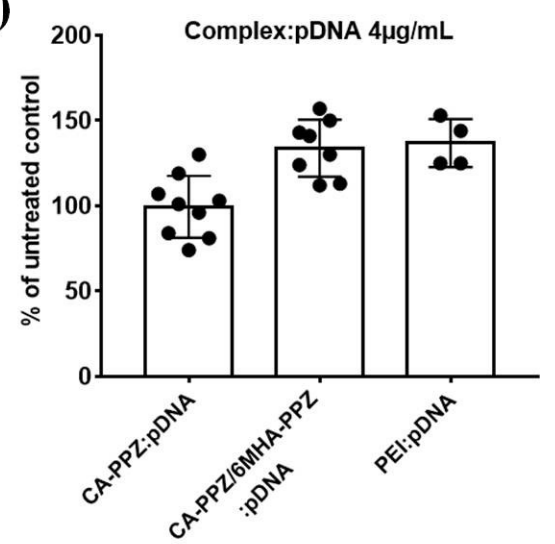

Figure S8. Cytotoxicity of polyphosphazene complex was evaluated by spheroid volume and metabolic activity in U87MG spheroids. Initial spheroid diameter was $\sim 250 \mu \mathrm{m}$ at day $0\left(\mathrm{t}_{0}\right)$ and was measured over time after treatment with polymer complexes at a) $2 \mu \mathrm{g} \mathrm{mL}^{-1}$ and b) 4 $\mu \mathrm{g} \mathrm{mL} \mathrm{m}^{-1}$ of pDNA concentrations $(\mathrm{n} \geq 4)$. Metabolic activity of U87MG spheroids treated with polymer complexes at c) $2 \mu \mathrm{g} \mathrm{mL}^{-1}$ and d) $4 \mu \mathrm{g} \mathrm{mL} \mathrm{m}^{-1}$ of pDNA concentrations, measured via resazurin reduction. Data is normalized to spheroids cultured in untreated cell media and presented by mean $\pm \operatorname{SEM}(\mathrm{n} \geq 4)$. 


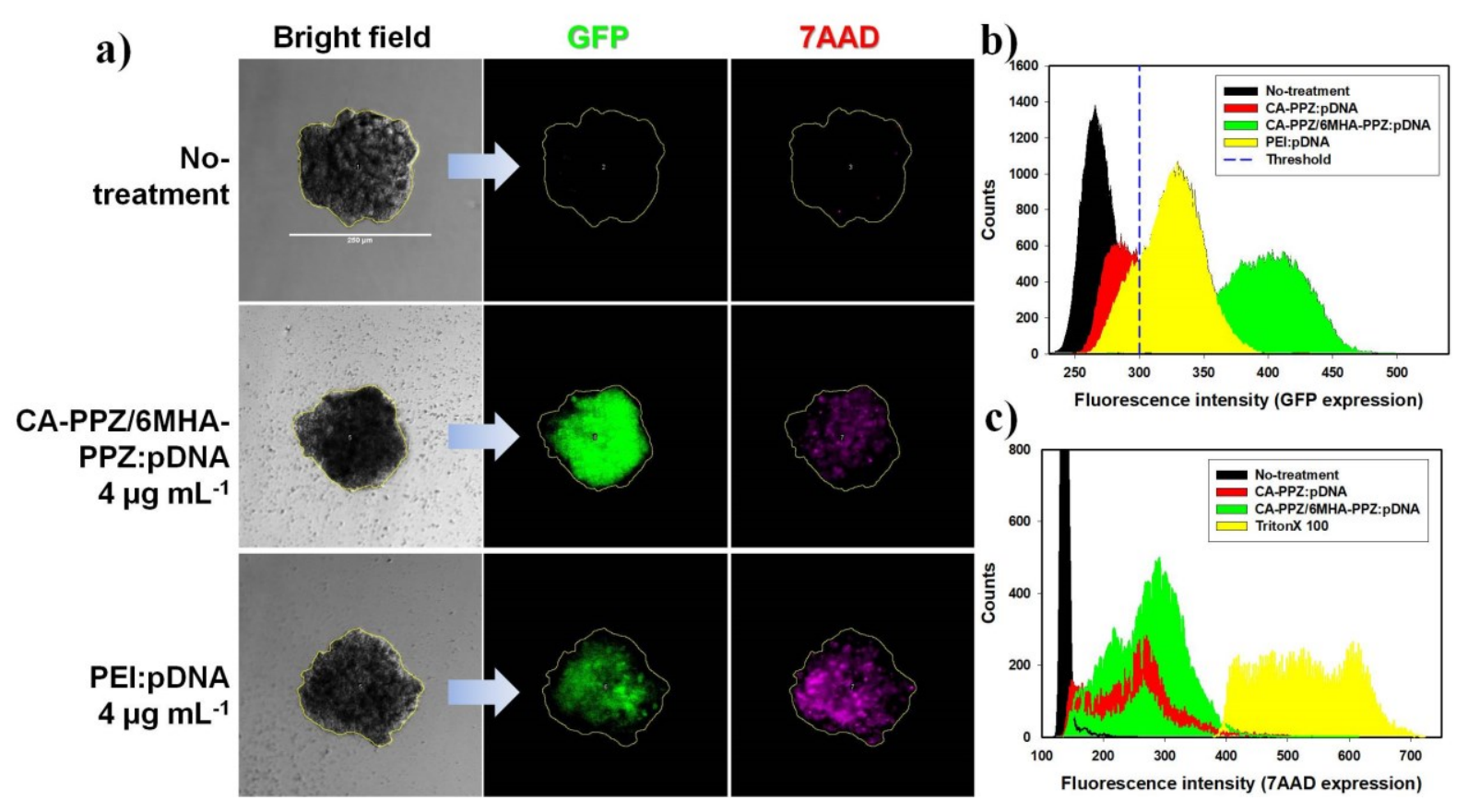

Figure S9. Diagrams showing the region-of-interest (ROI) selection in spheroid areas (a) and corresponding analysis via GFP (b) and 7AAD (c) fluorescent intensity histograms of spheroids treated with polymeric complexes with pDNA $4 \mu \mathrm{g} \mathrm{mL} \mathrm{m}^{-1}$.

Note: 7AAD fluorescent peaks of PEI complexes are considerably overlapped with CA-PPZ and CA-PPZ/6MHA-PPZ complexes but slightly higher than both PPZ-complexes (data not shown). 


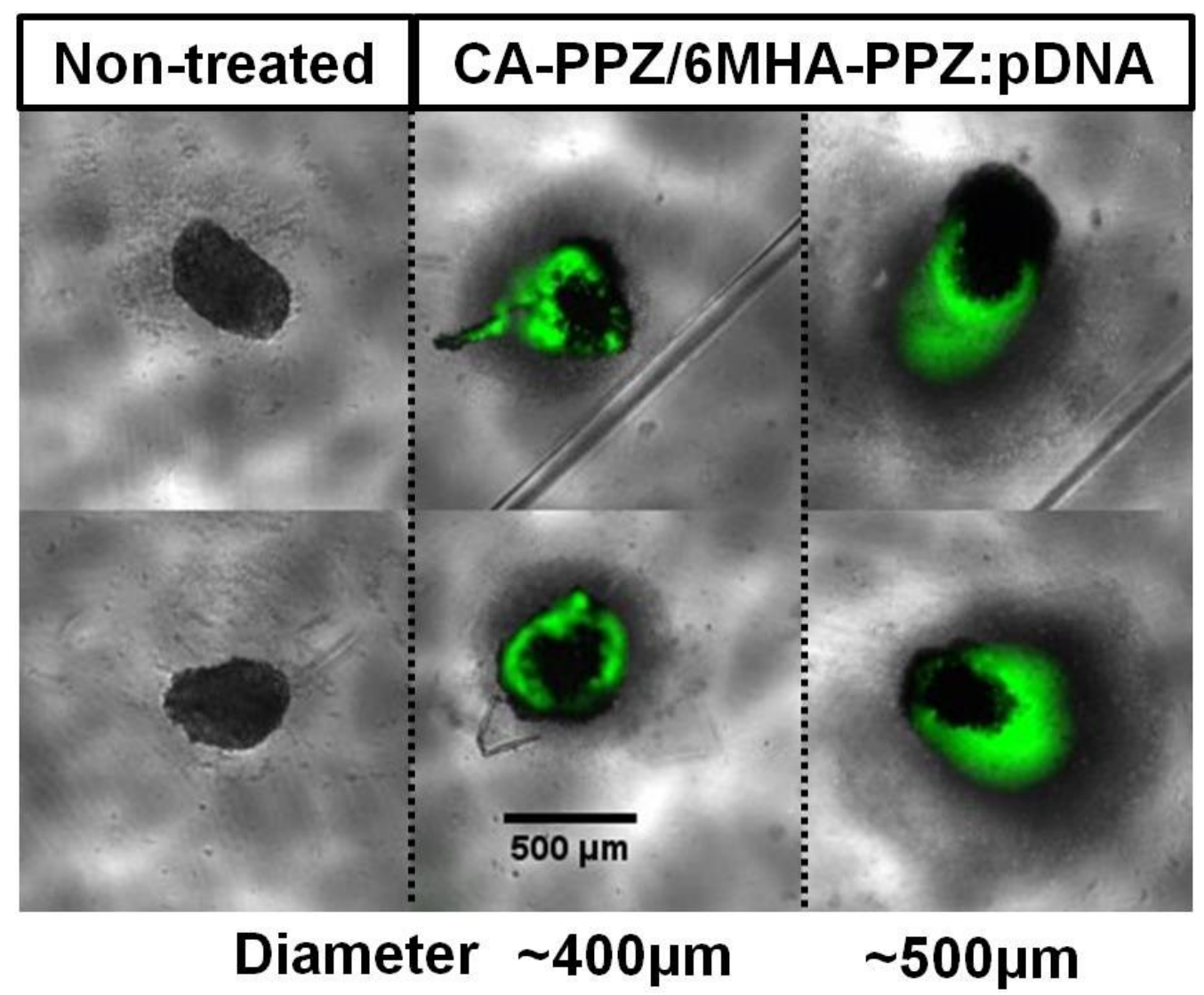

Figure S10. Transfection by CA-PPZ/6MHA-PPZ complexes carrying GFP-encoding plasmid in large spheroids (Diameter $\geq 400 \mu \mathrm{m})$. 
a)

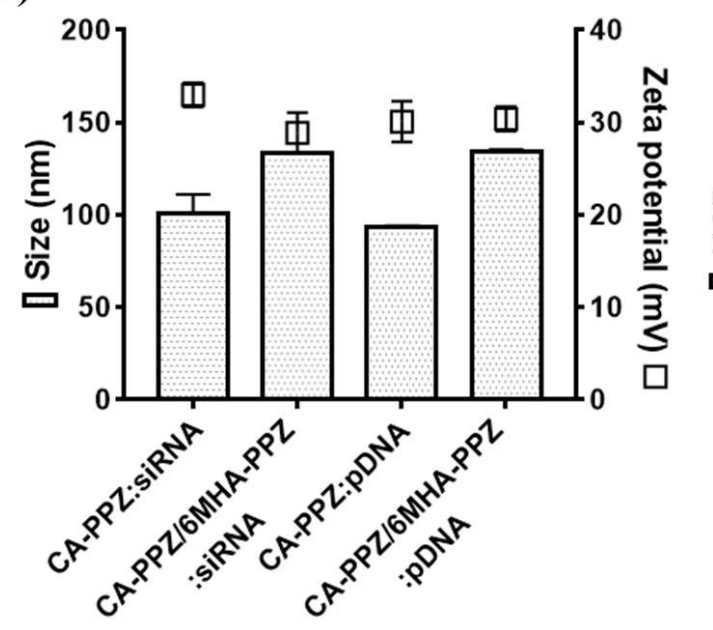

b)

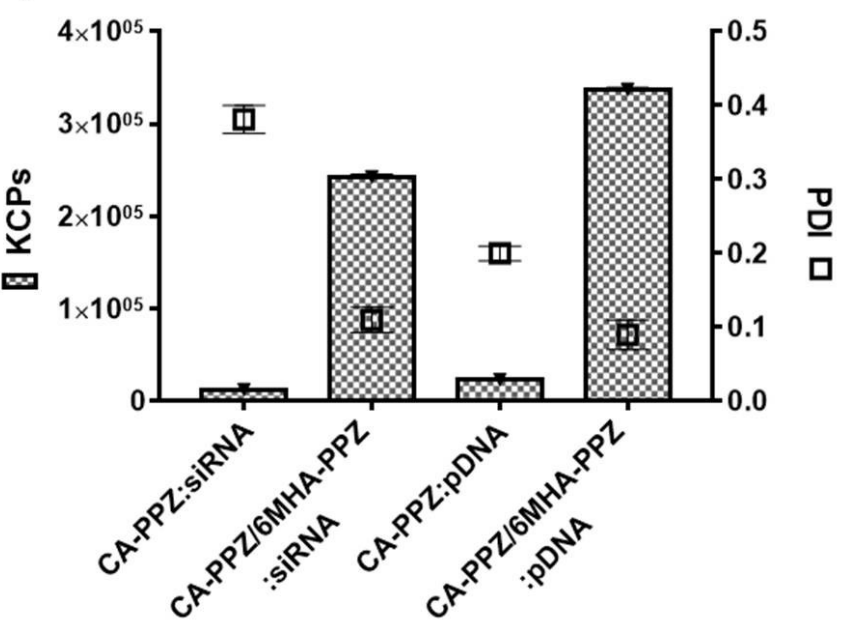

Figure S11. Comparison of particle characterization data for CA-PPZ complexes and CA-PPZ/6MHA-PPZ complexes carrying either pDNA or siRNA. Particle size and surface charge are shown in a); total derived count (KCPs) and polydispersity index (PDI) are shown in b). Data presented by mean $\pm \mathrm{SD}(\mathrm{n}=3)$. 
Table S1. Apparent pKa and change in the percentage of protonated groups between $\mathrm{pH} 7.4$ and 6.0 for the anionic polyphosphazenes. Data derived from potentiometric titrations and presented by mean $\pm \operatorname{SD}(n=3)-$.

\begin{tabular}{|c|c|c|c|c|}
\hline Polyanion & 3MPA-PPZ & 4MBA-PPZ & 6МHA-PPZ & 8MOA-PPZ \\
\hline Apparent $\mathrm{pKa}$ & $5.56 \pm 0.09$ & $5.89 \pm 0.07$ & $6.55 \pm 0.08$ & $7.14 \pm 0.10$ \\
\hline$\triangle \alpha(\mathrm{pH} 7.4-6.0)$ & $19.7 \% \pm 3.2 \%$ & $29.1 \% \pm 4.0 \%$ & $61.9 \% \pm 3.6 \%$ & $29.7 \% \pm 2.6 \%$ \\
\hline
\end{tabular}

\section{Supplementary videos}

Three videos are also uploaded as part of the supplementary information. The videos show tomographic images of $3 \mathrm{D}$ spheroids treated with the complexes loaded with a pDNA encoding GFP. Blue channel is for DAPI staining and green for GFP. Videos correspond to spheroids treated with: (i) buffer ("Control tomography"), (ii) CA-PPZ complexes ("CA-PPZ tomography”), and (iii) CA-PPZ/6MHA-PPZ complexes (“CA-PPZ_6MHA-PPZ tomography"). 


\section{Appendix: pKa calculation and protonation degree calculation}

$\mathrm{pKa}$ calculation was performed by Henderson-Hasselbalch equation (Equation S1) based on the potentiometric titration curves (Figure S3). The titration curve data were re-plotted to scatter diagrams by $\mathrm{pH}$ against $\log \left(\left[\mathrm{A}^{-}\right] /[\mathrm{HA}]\right)$, and $\mathrm{pKa}$ was obtained by a linear fitting between $\mathrm{pH} 8$ and $\mathrm{pH} 5$.

$$
p H=p K a+\log \left(\frac{\left[A^{-}\right]}{[H A]}\right)
$$

Protonation degree, alpha $(\alpha)$ represents the percentage of protonated carboxylic acid groups in the whole anionic polyphosphazene, and the calculation of $\alpha$ is shown below ${ }^{[1]}$ :

$$
\alpha=\frac{C_{p}}{C_{A}}=\sum_{i=0}^{n}\left(C_{H C l}-\mathrm{C}_{\left[\mathrm{H}^{+}\right]}\right)-\mathrm{C}_{\left[\mathrm{OH}^{-}\right]} / C_{A}
$$

Note 1. $C_{A}$ denotes total concentration of alkyl-carboxylic side groups in the polyanionic solution; $C_{p}$ denotes the concentration of protonated alkyl-carboxylic acid groups; $C_{H C l}$ denotes each addition of $\mathrm{HCl}$ concentration; $\mathrm{C}_{\left[\mathrm{H}^{+}\right]}$is the concentrations of free $\left[\mathrm{H}^{+}\right]$, which can be measured by $\mathrm{pH}$ meter, and $\mathrm{C}_{[\mathrm{OH}-]}$ is the initial concentration of $\left[\mathrm{OH}^{-}\right]$in the test solution, adjusted by excess $\mathrm{NaOH}$ for ensuring that all polyanions have been deprotonated; $i=0$ is the initial point, where we defined $0 \%$ as the protonated alkyl-carboxylic acid groups around $\mathrm{pH} 11$; $n$ is the number of $\mathrm{HCl}$ additions.

Note 2. There is an assumption that the total volume is kept constant during the titration (the volume percentage of each addition of $\mathrm{HCl}$ is 4/1000 of total volume). 


\section{Reference}

[1] a) G. J. Koper, R. C. van Duijvenbode, D. D. Stam, U. Steuerle, M. Borkovec, Macromolecules 2003, 36, 2500; b) R. C. van Duijvenbode, M. Borkovec, G. J. Koper, Polymer 1998, 39, 2657; c) R. Smits, G. Koper, M. Mandel, The Journal of Physical Chemistry 1993, 97, 5745. 


\title{
Polyphosphazenes for the delivery of biopharmaceuticals
}

\author{
Wei-Hsin $\mathrm{Hsu}^{1,2}$, Noemi Csaba ${ }^{1}$, Cameron Alexander ${ }^{2}$, Marcos Garcia-Fuentes ${ }^{1 *}$ \\ ${ }^{1}$ Center for Research in Molecular Medicine and Chronic Diseases (CiMUS), Universidade de \\ Santiago de Compostela \\ ${ }^{2}$ Division of Molecular Therapeutics and Formulation School of Pharmacy, University of \\ Nottingham, UK \\ * Corresponding author: marcos.garcia@usc.es
}

\author{
Abbreviation: \\ Polyphosphazene (PPZ), Poly(dichlorophosphazene) (PDCP) \\ Poly[di(carboxylatophenoxy)-phosphazene] (PCPP), Poly[di(sodium \\ carboxylatoethylphenoxy)phosphazene] (PCEP) \\ Polylactide (PLA), Poly(lactide-co-glycolide) (PLGA), Polycaprolactone (PCL), \\ Polyethylenimine (PEI) \\ Diisopropyl ethylenediamine (DPA), 2-dimethylaminoethanol (DMAE), 2- \\ dimethylaminoethylamine (DMAEA), N,N-diisopropylethylenediamine (DPA), \\ Carboxylatoethylphenoxy (CEP) \\ Lower critical solution temperature behavior (LCST) \\ Human growth hormone (hGH) \\ Aluminum-based mineral salts (Alum), Hepatitis B surface antigen (HBsAg) \\ Type 1 T helper (Th1), Type 2 T helper (Th2), Dendritic cells (DCs)
}




\begin{abstract}
Polyphosphazenes (PPZs) are a relatively new family of polymers based on a nitrogenphosphorous backbone where organic side-groups can be grafted. The synthetic route to PPZs is highly versatile such that it is possible to add many different functionalities that change completely the physicochemical and biological properties of the polymers. For instance, PPZs can be designed with a variety of organic side groups that render these materials biodegradable and highly biocompatible. Based on these positive features, PPZs have been explored for many biomedical applications including the design of numerous advanced drug delivery systems. In this area, PPZs have been particularly investigated as materials for the formulation of biopharmaceuticals of high added value. These include protein- and polynucleotide-based medicines, applications where PPZ carriers have obtained very positive results in pre-clinical models. A further area of major interest for PPZs has been vaccination, where these materials have obtained excellent results in vivo as polymer adjuvants and have advanced to clinical evaluation.
\end{abstract}




\section{Part I. Background and fundamental properties of PPZ}

New biodegradable materials are required in many biomedical applications, and polymers continue to be critical for this field. In comparison to natural polymers, synthetic biodegradable polymers are generally better defined and are easier to modulate regarding their mechanical and degradation properties. Polyesters such as polylactide (PLA), poly(lactide-co-glycolide) (PLGA), or polycaprolactone (PCL) are some of the most used synthetic polymers, but other polymer families such as polyphosphazenes (PPZs) are also of interest since they bring advantages of tunable biodegradability, polymer elasticity and chemical versatility. Due to these characteristics, polyphosphazenes are being investigated in a range of biomedical applications such as controlled drug delivery and tissue engineering ${ }^{1,2}$.

Polyphosphazenes (PPZs) are a class of polymers having an inorganic backbone made of repeating units of phosphorus and nitrogen in an alternating sequence, and which can be configured in cyclic or linear conformations. The repeating unit of PPZs is $N=\mathrm{PR}_{1} \mathrm{R}_{2}$ (Figure 1a), a structure built by phosphorus covalently linked to nitrogen via alternating $\sigma-\sigma$ bond and $\sigma$ $\pi$ bonds. Each phosphorus atom offers five electrons and each nitrogen provides another five electrons, forming sequential saturated and unsaturated bonds on the backbones. Within this structure two electrons of the phosphorus are used for side chain conjugation, and two electrons of nitrogen remained as a lone-pair (Figure 1b). Although the linear backbone contains unsaturated bonds, the $d \pi(\mathrm{P})-p \pi(\mathrm{N})$ bond is expected to have flexible rotation because several $3 d$ orbitals of phosphorus can hybridise with the $p_{z}$ orbital of nitrogen once the $\pi$ bond undergoes torsions (Figure 1c) ${ }^{3,4}$. As theoretical calculations report, the bond energy of inherent torsional barrier in the phosphazene backbone is as low as 100 cal per bond ${ }^{4,5}$. Nevertheless, the linear PPZ conformation is significantly influenced by their side groups (i.e. $\mathrm{Cl}, \mathrm{OCH}_{2} \mathrm{CF}_{3}$, etc.), since the phosphazene skeleton is easily distorted and lies preferably in a cis-trans rather than in a trans-trans planar conformation to minimize internal repulsions (Figure 1d) ${ }^{3,6}$. Because of the flexible $d \pi(\mathrm{P})-p \pi(\mathrm{N})$ bond, most PPZ are colorless, have insulating properties and lack microcrystallinity. This is more apparent in PPZs having two or more different substituting side groups ${ }^{4}$.

The most commonly prepared PPZ is poly(dichlorophosphazene) (PDCP), which is synthesized as a precursor for its derivation to a wide range of polymers by nucleophilic substitution ${ }^{7}$. Many 
PDCP derivatives have been synthesized and formulated to various products in biomedical applications. For example, amino acid ester PPZs have been widely studied and applied to prepare fibers or scaffolds by electrospinning for bone tissue engineering ${ }^{8,9}$; amphiphilic PPZs have been used for the preparation of nanoscale polymeric carriers in controlled drug delivery 10,11. For vaccine delivery the most common prototypes are based on PPZs with carboxylic acids side chains, such as poly[di(carboxylatophenoxy)-phosphazene] (PCPP). The versatility of PPZ chemistry is also important from scale-up and manufacture perspectives ${ }^{12-14}$. For many industrial applications, it is desirable to have 'platform' materials that can be manufactured in bulk and adapted or modified for specific purposes. For pharmaceutical applications, a single precursor polymer that can be tailored for different therapies would be highly advantageous. In this review we focus on linear PDCP derivatives and their bio-applications in gene delivery, vaccine delivery and protein drug delivery.

a)

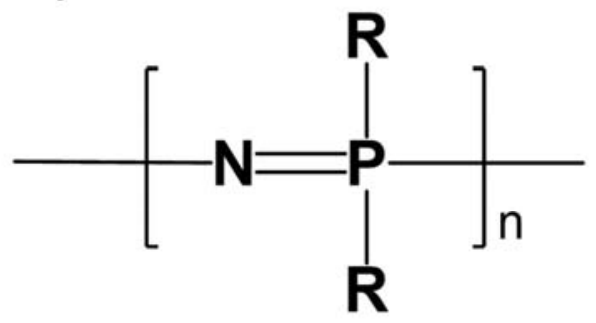

b)

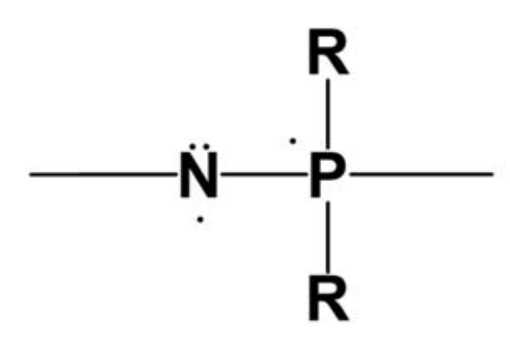

c)

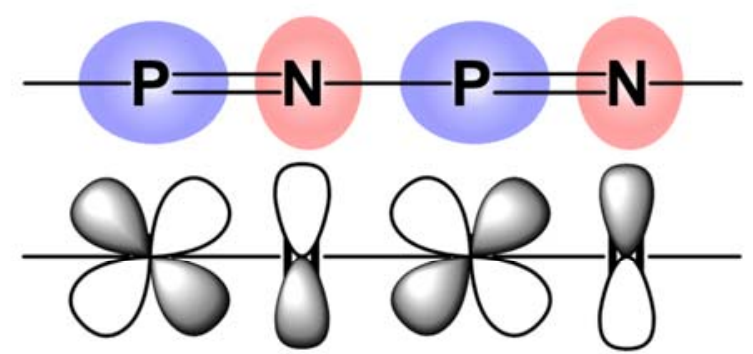

d)

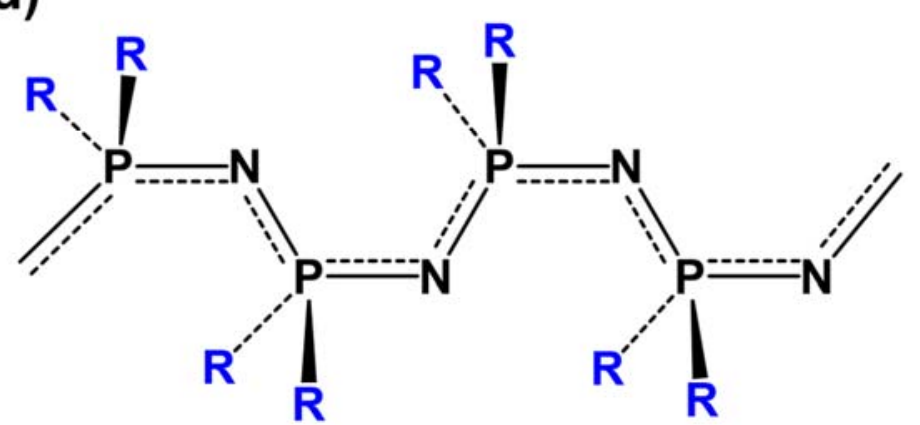

Figure 1. General structures of PPZ (a), electron arrangement in the phosphazene bond (b) and its orbital hybridization (c), in which phosphorus provides $\mathrm{d}_{x z}$ to hybrid with $\mathrm{p}_{z}$ of nitrogen. (d) Cis-trans planar conformation of repeating phosphazene backbones. 


\section{Synthesis of polyphosphazene and functional additions}

The first synthesis of PPZ was reported by Stokes as early as 1897 in a high-temperature polymerization reaction that led into an insoluble elastomer known as inorganic rubber. The first stable synthesis of PPZ was reported by Allcock and co-workers ${ }^{15}$, a work that resulted in increased interest for these kind of materials for biomedical and other applications. The scheme developed by Allcock is still the basis of the most general procedure for linear PPZ synthesis: the precursor poly(dichlorophosphazene) (PDCP) is prepared in the first step, and then the final polymer is formed by nucleophilic substitution of the desired side chains ${ }^{1,16,17}$.

Based on the raw monomers used to prepare linear PDCP, synthetic methods can generally be classified based on those starting from cyclic trimers (i.e. hexachlorocyclotriphosphazene, $\left.\left(\mathrm{NPCl}_{2}\right)_{3}\right)^{15,18}$, and those starting from non-cyclic monomers (i.e. dichlorophosphinoyliminotrichloro phosphorene, $\mathrm{Cl}_{3} \mathrm{P}=\mathrm{N}-\mathrm{P}(\mathrm{O}) \mathrm{Cl}_{2}$, or trichloro(trimethylsilyl)phosphoranimine, $\mathrm{Cl}_{3} \mathrm{P}=\mathrm{NSiMe}_{3}{ }^{19-22}$. In the former case, the polymerization is typically thermo-initiated and the ring-opening reaction propagates towards linear PDCP (Figure 2a). Many aspects of this general PDCP synthesis procedure have been improved, including catalyzed polymerization ${ }^{23,24}$ and solvent-mediated stabilization ${ }^{25}$. Although the mechanism of this ring-opening polymerization is still open to debate, the most broadly accepted mechanism is that one phosphorus-chloride bond of the cyclic trimer is cleaved by heating above $250-260{ }^{\circ} \mathrm{C}$, triggering the opening of the next cyclotriphosphazene and starting the chain propagation reaction. In the terminal step, the reactive head of the PPZ chain $\left(\sim \mathrm{N}=\mathrm{PCl}_{2}^{+}\right)$can recapture the chloride anion as the reaction temperature decreases. This reaction is extremely sensitive to contamination by water and other nucleophiles, which can cause unwanted cross-linking, and precipitation of an insoluble material (i.e. Stokes' "inorganic rubber") as well as limiting the reproducibility of the synthesis. For avoiding water contamination, a solvent-free melt polymerization reaction can be used, but this reaction is difficult to control and forms ultra-high molecular weight PDCP.

The use of efficient catalysts is a critical factor to achieve high-yield, controllable polymerization of PDCP and to obtain a product with sufficient purity to proceed for side change substitution reactions without further purification ${ }^{23,24}$. Boron trichloride $\left(\mathrm{BCl}_{3}\right)$ and aluminum chloride $\left(\mathrm{AlCl}_{3}\right)$ are strong Lewis acids that facilitate the extraction of the chloride on the trimers and act as catalysts for cyclic trimer activation. $\mathrm{BCl}_{3}$ has the additional advantage of reducing the 
possibility of crosslinking by eliminating trace amounts of water in the polymerization by forming $\mathrm{B}(\mathrm{OH})_{3}{ }^{23}$. Sohn et al. also reported that $\mathrm{AlCl}_{3}$ can be used as a catalyst in PPZ polymerization to yield PDCP with a mass average molecular weight $\left(\mathrm{M}_{\mathrm{w}}\right)$ range between $10 \mathrm{kDa}$ and $100 \mathrm{k} \mathrm{Da}{ }^{24}$, which is preferred for many biomedical applications, especially drug delivery and tissue engineering ${ }^{26,27}$.

Besides these methods relying on ring-opening polymerization starting from hexachlorocyclotriphosphazene, PDCP can also be synthesized through phosphorus pentachloride $\left(\mathrm{PCl}_{5}\right)$ initiated polymerization starting from non-cyclic monomers, such as trichloro(trimethylsilyl)phosphoranimine, $\mathrm{Cl}_{3} \mathrm{P}=\mathrm{N}-\mathrm{Si}\left(\mathrm{CH}_{3}\right)_{3}$ (Figure 2b) ${ }^{19,20,22}$. This method has several advantages such as room-temperature synthesis and controllable molecular weights with narrow polydispersity. Most interestingly, this polymerization method can be used to prepare further block copolymers, such as PLA-co-PDCP, PEO-co-PDCP and polystyrene-co-PDCP ${ }^{28-}$ 30. The first section of polymer with terminal primary amines can conjugate with $\left(\mathrm{CF}_{3} \mathrm{CH}_{2} \mathrm{O}\right)_{2} \mathrm{BrP}=\mathrm{NSiMe}_{3}$ by nucleophilic substitution and then react with $\mathrm{PCl}_{5}$ to initiate the polymerization of the PDCP block. In light of this, $\mathrm{PCl}_{5}$-induced living cationic polymerization provides broader possibilities for PPZ platforms, such as the preparation of amphiphilic polymers or multi-arm branch polymers, which could be used in medical applications. 
a)

Initiation

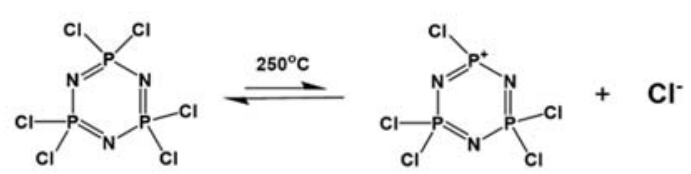

roperamen

$(n / 3)$

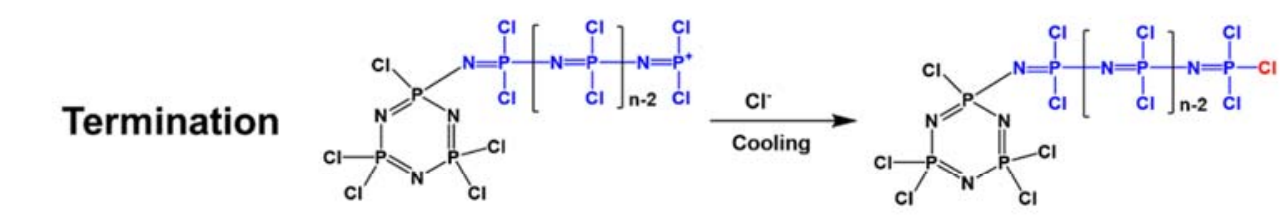

b)

Initiation

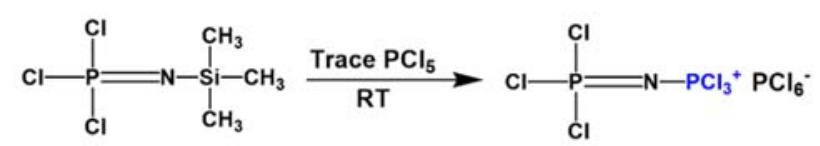

Propagation

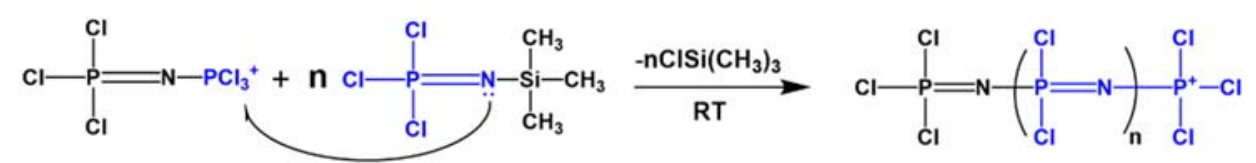

Termination

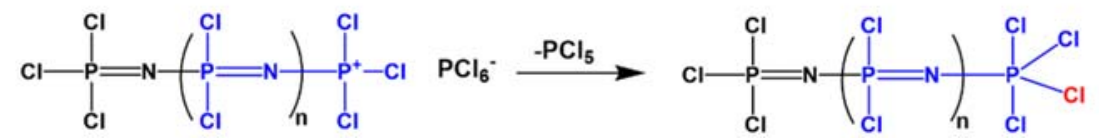

Figure 2. Potential mechanisms for PPZ polymerization. (a) Ring-opening polymerization of cyclotriphosphazene and (b) living cationic polymerization of trichloro(trimethylsilyl)phosphoranimine to yield linear poly(dichlorophosphazene)s (PDCP).

Despite all the work on improving its synthesis, PDCP has few applications itself, but rather acts as a precursor polymer that, to date, has been modified into over 700 different derivatives ${ }^{4,7}$. Indeed, PDCP provides an easy-coupling platform for conjugation with side-chain candidates, in which nucleophile terminals can substitute the chlorines of this polymer, effectively conjugating to the PPZ backbone. Despite the flexibility of this reaction, the nucleophilic substitution is limited to functional groups having only one nucleophilic center. If groups having more than one 
nucleophilic center are used, a crosslinking reaction and the precipitation of the polymer intermediate will result. Therefore, many functional groups of biological interest (amines, acids, or saccharide molecules etc.) need to go through protection/deprotection procedures for their nucleophilic substitution on PDCP. Fortunately, an extensive library of PPZs has been developed, including derivatives substituted with alkene, alkyne, or vinyl groups, for example, allylamine 14,31, 2-aminoethyl methacrylate ${ }^{32}$, allyl glycinate ${ }^{33}$, methacrylic acid ${ }^{34,35}$ and propargylamine 36 derivatives. These PPZs with alkene and alkyne side chains can be used as secondary

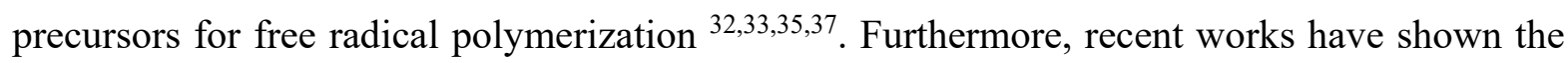
possibility to use these groups in thiol-ene, thiol-yne and azide/alkyne click reactions as simple pathways for PPZ derivatization with biomolecules ${ }^{14,36,38,39}$. These reactions have also been applied as simple schemes to add ionic side chains on the PPZ backbone for the design of gene therapy polymers ${ }^{40}$. Overall, free-radical polymerization and click reactions bring additional versatility to PPZs and allow for the introduction of potential biomolecules or therapeutic compounds as side chains.

\section{Biocompatibility and Biodegradability of polyphosphazenes}

Poly(organophosphazenes) is a term for PPZs having organic side groups. These polymers cover a broad range of materials with tunable cytocompatibility and biodegradability ${ }^{7,41}$, and thus the following discussion is centered on these PPZs. A considerable number of organic side chains have been introduced in PPZs, including amino acid esters ${ }^{7,42-44}$, peptides ${ }^{33,42,45}$, saccharides 14,36,46, arylcarboxylates ${ }^{47}$, ethylene oxide/PEG ${ }^{11,27,48}$, and other biomolecules (purine and pyrimidine bases, vitamins, etc.) ${ }^{49,50}$. For biomedical applications, the safety of these materials is a key consideration. As with other properties of polyphosphazenes, the cytocompatibility of the final materials is dictated by the side groups used. For instance, polyphosphazenes substituted with tertiary amines can exert certain toxicity even at moderate concentrations ${ }^{51}$. On the other hand, other polyphosphazenes used in bone tissue engineering show excellent safety profiles ${ }^{9}$. For instance, poly[(ethyl glycinato) phosphazene] has been compared to PLGA in a cytocompatibility test performed in primary rat osteoblasts, where this PPZ showed no negative effect on cell proliferation ${ }^{52}$. This study also inspired further tests of alanine-based PPZs regarding osteocompatibility. This material showed low toxicity and good capacity to support 
cell adhesion, proliferation, and maintenance of cellular phenotype ${ }^{53,54}$. Other types of PEGylated PPZs also show excellent biosafety profiles that combine with their antifouling properties ${ }^{55-57}$. The biocompatibility of the PPZ backbone has also been supported by the FDAapproval of Cobra PzF stents (CeloNova Biosciences Inc.) in 2017, which are coronary stents coated with nanostructured PPZs for thrombo-resistant properties ${ }^{58}$.

A critical characteristic of poly(organophosphazenes) is their capacity to biodegrade, which is beneficial in many medical applications. The mechanism for hydrolysis of these PPZs is still controversial and three possible mechanisms have been suggested ${ }^{41,59,60}$. In mechanisms 1 and 2 (see Figure 3), the side groups can react with the phosphazene backbone and accelerate polymer hydrolysis. In the other mechanism, the side chains of PPZs may be eliminated from their backbone first, and finally the backbone can be hydrolyzed. In all cases, the result of the hydrolysis is the formation of degradation products from the side groups and a self-neutralizing buffer of ammonium phosphate derived from the backbone ${ }^{7,43}$. The neutrality of these degradation products is another advantage over other biodegradable polymers that form acid residues that can harm delicate biomolecules ${ }^{61,62}$.

The degradation rate of PPZs is also dependent on the substituting side groups and their ratios when there is more than one type ${ }^{63}$. Within this regard, important characteristics of the side groups are: hydrophobicity, steric hindrance, and the degree of crosslinking of the side chains. The lack of systematic studies has made it difficult to compare a broad range of side chains under standardized conditions ${ }^{7}$. However, some amino-acid ester PPZs have been studied in detail by the group of Allcock, where they calculated their degradation half-life $\left(\mathrm{T}_{1 / 2}\right)$ in PBS $37{ }^{\circ} \mathrm{C}$ as follows: ethyl glycinate $\left(\mathrm{T}_{1 / 2} \sim 3\right.$ months $)<$ alanine $\left(\mathrm{T}_{1 / 2} \sim 6\right.$ months $)<$ valine $\left(\mathrm{T}_{1 / 2} \sim 1\right.$ year $) \leqq$ phenylalanine ethyl ester $\left(\mathrm{T}_{1 / 2} \sim 1\right.$ year) ${ }^{43,44,64}$. Moreover, when analyzing two water-soluble PPZs with the same terminal functional side group (i.e. a tertiary amine), but linked to the PPZ backbone by a different nucleophile group, $-\mathrm{N}=\mathrm{P}\left(-\mathrm{OCH}_{2} \mathrm{CH}_{2} \mathrm{~N}\left(\mathrm{CH}_{3}\right)_{2}\right)_{2}$ vs. $\quad-\mathrm{N}=\mathrm{P}(-$ $\left.\mathbf{N H C H}_{2} \mathrm{CH}_{2} \mathrm{~N}\left(\mathrm{CH}_{3}\right)_{2}\right)_{2}$, the authors found that the alkoxide-substituted PPZ degraded faster $\left(\mathrm{T}_{1 / 2}\right.$ $\sim 7$ days) than the amine-substituted PPZ (T/2 $\sim 24$ days $)^{51}$.

In conclusion, the good cytocompatibility profile of poly(organophosphazene)s together with their biodegradation kinetics that can be tuned by selecting appropriate side groups is another 
positive characteristics that indicates the interest of these materials for tissue engineering and drug delivery $9,41,65,66$.
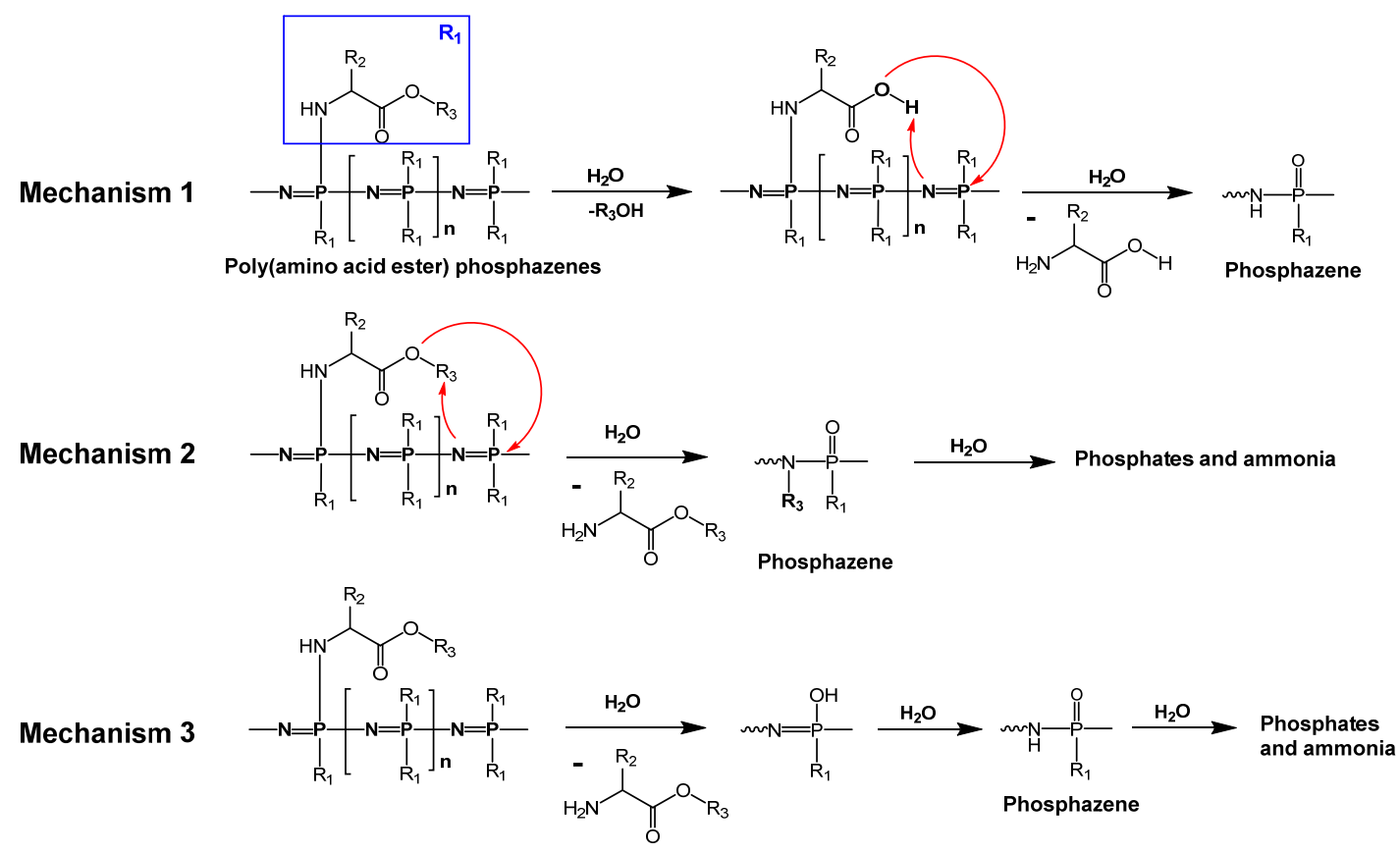

Figure 3. The three common hypotheses of poly(amino-acid ester)phosphazenes for degradation mechanisms. [Adapted from Allcock, H. R. et al. 2012, Polymer Chemistry ${ }^{7}$, with permission]. 


\section{Part II. Use of PPZs for the delivery of biomacromolecules}

Macromolecular drugs (polysaccharides, protein, nucleotide, etc.) are increasingly important in the clinical arsenal and are likely to be the main class of therapeutics in many future disease treatments. However, most biomacromolecules have low stability in the body and lack the capacity to cross biological barriers. New carriers to improve the delivery of macromolecules are thus urgently needed. PPZ-based platforms combine chemical flexibility, biodegradability and biocompatibility, suitable characteristics that suggest their use for macromolecule carrier design. Here we review the use of PPZ platforms for gene and protein delivery; afterwards, we cover specifically the use of PPZs for protein and gene delivery in vaccination, since this application has particular technical requirements.

\section{Gene delivery}

Gene therapies are some of the most promising advanced treatments under investigation today, and they have already resulted in some new medicines translated to the clinic. The major bottleneck towards the successful use of DNA and RNA is their delivery to the target tissues as well as to their target cell compartments. This delivery problem results in poor efficacy/toxicity ratios and has slowed the development of gene therapy for decades ${ }^{67-69}$. In polymer-mediated gene delivery, the most widely-investigated material is polyethylenimine (PEI), whose positively charged amine groups can condense polynucleotides leading to the formation of nanocomplexes. PEI-nucleotide polyelectrolyte complexes can be taken up by cells and the buffering capacity of the PEI backbone amines can facilitate their escape from endosomal compartments, effectively improving the intracellular delivery of the gene medicines ${ }^{70}$. However, PEI has important limitations regarding its medical use: it is non-biodegradable and has detrimental effects related to mitochondrial dysfunction ${ }^{71-73}$, thus better alternative polymers are sought as gene delivery carriers.

The first PPZ employed for the purpose of gene delivery was reported by Hennink's group ${ }^{51}$, who compared the degradation ratios of two terminal tertiary amines as PPZ side chains, 2dimethylaminoethanol (DMAE) and 2-dimethylaminoethylamine (DMAEA). The corresponding 
cationic polymers were used to complex plasmid DNA for gene delivery (Figure 4). The studies indicated that DMAEA-PPZ complexing DNA has higher gene transfer efficiency than DMAEPPZ in the COS-7 cell-line, especially at low N/P ratios $\leq 10$ (the molar ratio of pronated amine group to negative phosphate of nucleotide). However, DMAEA-PPZ has higher toxicity than DMAE-PPZ at high N/P ratios ( $\geq 20$ ), likely due to the short half-life of DMEA-PPZ. Besides, these authors also investigated the effect of DMAEA-PPZs molecular weight on DNA delivery efficiency. The lower molecular-weight polymers $\left(\mathrm{M}_{\mathrm{w}}\right.$ range of $\left.130-950 \mathrm{k} \mathrm{Da}\right)$ resulted in acceptable toxicity both in vitro and in vivo ${ }^{74}$. Further improvements in these early prototypes were achieved by introducing imidazole groups in DMAEA-PPZs as added functionalities to improve endosomal escape and enhance transfection efficiency ${ }^{75,76}$. Other chemical modifications of PPZ included grafting with galactose ${ }^{77}$ and PEG ${ }^{78}$ to improve biocompatibility and stability of the drug carriers.

These initial designs were mostly limited to grafting PPZs with groups having only one nucleophilic center due to synthetic limitations related to uncontrolled polymer crosslinking that could only be avoided by cumbersome protection/de-protection procedures ${ }^{79-81}$. For addressing this issue, Hsu et al. used a click-addition extension starting from a PPZ backbone (Figure 4) and applied this synthetic strategy to the design of a small library of materials for gene delivery that included previously unexplored primary amine PPZs and carboxylic acid PPZs of medium/low molecular weight ranges $(10 \mathrm{kDa} \text { to } 50 \mathrm{kDa})^{40}$. Primary amine PPZs were found to be more efficient for gene delivery than tertiary amine PPZs due to their higher binding capacity for DNA. Additionally, the authors identified a carboxylic acid functional grafting group that could be added to the gene nanocarriers as a delivery enhancer. This material not only reduced the toxicity on the nanocarriers, but improved gene transfection in several 2D and 3D cell models, as well as in vivo. In perspective, cationic PPZ-based gene complexes show comparable gene delivery efficacy to commercial standards (PEI or Lipofectamine), but they tend to show lower cytotoxicity and have the additional advantage of being biodegradable $40,57,76,78,82$.

Standard polyelectrolyte complexes are not the only type of structures that have been tested for gene delivery. For instance, thermosensitive, injectable PPZ gels have been used for the controlled release of chitosan-graft PEI/DNA complexes or PEI-grafted PPZ/DNA complexes ${ }^{83}$. This type of system can be used for local administration to achieve sustained release of the 
polymer/gene complexes ${ }^{84,85}$. Other types of structures of interest are polymersomes and polymeric micelles. Qiu's group developed PEG-PPZ block copolymers where PPZs were substituted with hydrophobic N,N-diisopropylethylenediamine (DPA) groups. The copolymers were amphiphilic and could be formulated as polymersomes. When these polymersomes were loaded with microRNA (miR-200c), the nanomedicine was capable of inhibiting tumor progression in xenografted mice with a drug-resistant lung cancer model ${ }^{57}$. Polymersomes were also used to deliver plasmids coding for recombinant IL-12, a protein that can activate cytotoxic $\mathrm{T}$ lymphocytes and natural killer cells in antitumor immunotherapy, but which can also present severe side effects via standard intravenous administration ${ }^{86,87}$. The polymersome was able to deliver the plasmid to CT-26 tumors in mice upon intravenous administration. This delivery system prolonged the half-life of IL-12 in the tumor region and in serum, while they minimized the therapy adverse effects ${ }^{88}$. After the polymersome treatment, immune effector cells (CD8+ T cells, NK cells and NKT cells) were recruited in the tumor environment, and increased concentrations of IFN- $\gamma$ shortly after the treatment.

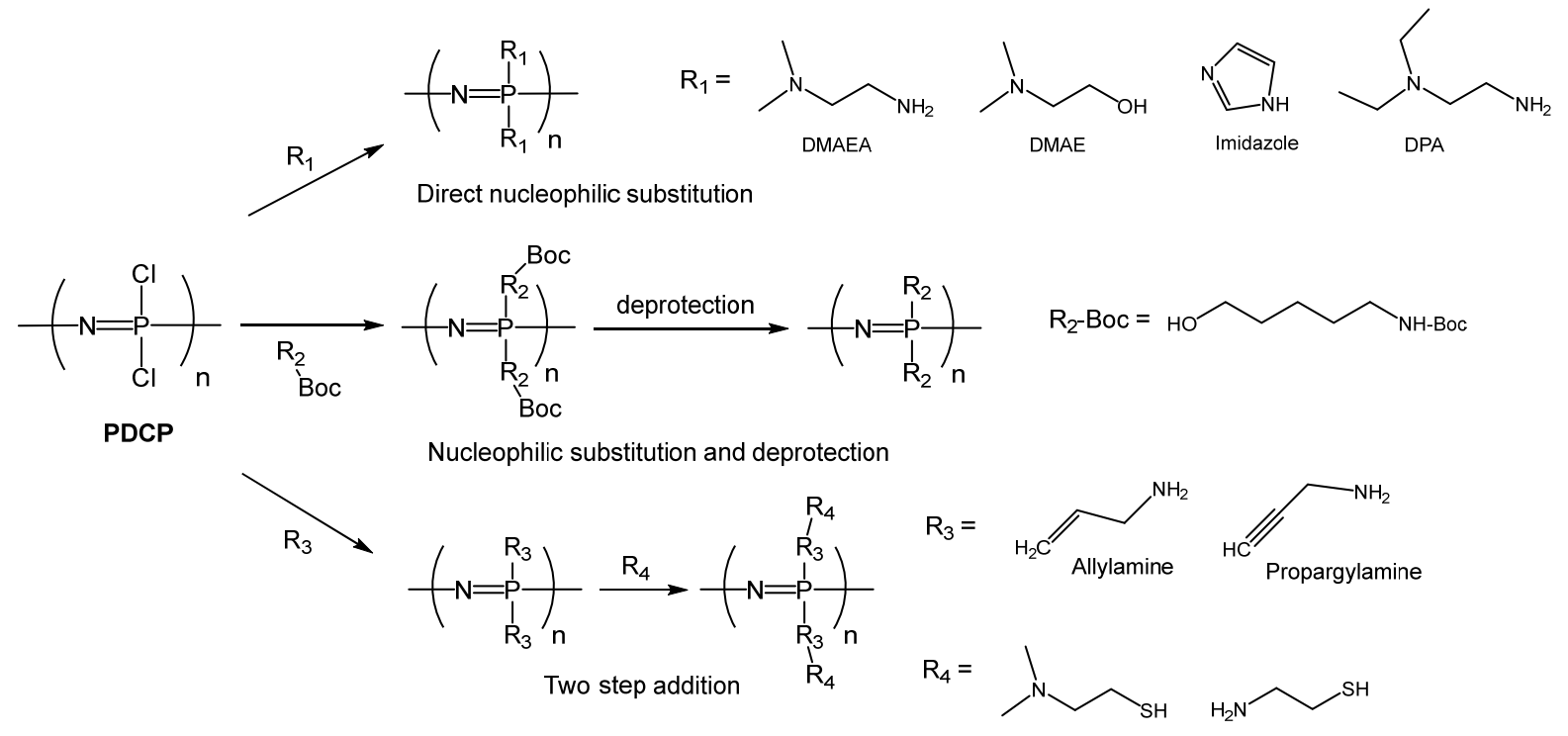

Figure 4. The common techniques to introduce cationic side chains on polyphosphazene (PPZ) backbone for gene delivery application.

\section{Protein drug delivery}


The possibility of generating materials with tailored degradation kinetics and their amenability to easily integrate other functionalities make poly(organophosphazene)s an attractive choice for protein delivery. Based on their general structure, we highlight two main strategies that have been followed in PPZ-based protein formulation: (i) nanoparticles and (ii) injectable gels.

Nanoparticles can be designed to provide protein stabilization and improved intracellular trafficking. For instance, the group of Andrianov presented an ionic polyphosphazene for "smart" protein delivery ${ }^{89}$. In this work, poly(carboxylatoethylphenoxy)-co-(3-(2-oxo-1-pyrrolidinyl)propylamino)phosphazene, PPA was designed with degradable/pH-sensitive functions. The optimized composition of PPA can induce hemolysis at endosomal $\mathrm{pH}$, facilitating endosomal escape after internalization in cancer cells. Carboxylatoethylphenoxy (CEP) side chains are wellknown for their use in complexation with proteins, but they are also sensitive to changes in $\mathrm{pH}$ within the physiological range. Complexation of L-Asparaginase with both negatively-charged CEP-grafted and positively-charged tertiary amine-grafted PEG-PPZ derivatives can form noncovalent PEGylated nanoassemblies for protein delivery; these nanocarriers promoted the stability of this enzyme and reduced their undesirable antigenicity ${ }^{55}$.

Since the short in vivo half-life of proteins is one of their most frequent shortcomings, injectable controlled release formulations are in great need. Non-ionic, thermosensitive PPZ polymers that gel at body temperature and provide sustained delivery of proteins are very promising in this regard. For instance, this type of hydrogel can be generated with PPZs grafted with three side chains corresponding to different functions: the hydrophobic L-isoleucine ethyl ester (IleOEt), the hydrophilic $\alpha$-amino- $\omega$-methoxy-poly(ethylene glycol) (AMPEG), and the hydrolysissensitive ethyl-2-(O-glycyl)lactate (GlyLacOEt). In this design, the ratio between IleOEt and AMPEG side groups define the lower critical solution temperature behavior (LCST), and thus the body temperature where the polymer will gel. GlyLacOEt moieties facilitate acid-catalyzed degradation and controlled protein drug release ${ }^{27,90}$. A composition of this polymer family capable of gelling at body temperature upon injection was used to formulate human growth hormone (hGH). This formulation formed a depot with sustained release properties that could address clinical issues associated with hGH treatments, such as renal toxicity and short half-life, which otherwise require multiple-injections leading to poor patient compliance ${ }^{91}$. Unfortunately, direct loading of hGH in this PPZ hydrogel still results in a large burst and fast release rates, 
which result in total cargo release in 1 week or less ${ }^{92,93}$. To improve this result, hGH was complexed with poly-L-arginine, and these biodegradable nanocomplexes then were loaded in the thermosensitive PPZ hydrogel. This multi-stage formulation could significantly prolong the release phase for several weeks. In another work, PPZs were designed to have dual interactions with proteins in order to generate more stable release profiles. The PPZs were grafted with hydrophilic PEG, hydrophobic isoleucine ethyl ester (IleOEt) and carboxylic acid moieties that could complex BMP-2 and form stable nanocomplexes. Compared to non-anionic amphiphilic PPZs complexes, anionic BMP-2 nanocarriers were able to duplicate the duration of BMP-2 release and avoid the burst effect. This formulation achieved continuous BMP-2 stimulation in vivo and resulted in osteocalcin secretion even two weeks after a single-dose injection of this nanocomplex. The treatment demonstrated new bone generation on mouse ectopic and orthotopic sites after 8 weeks ${ }^{94}$.

\section{Vaccine delivery}

Vaccination is currently the most cost-effective method for protection against infectious diseases. Generally, vaccines are derived from either live attenuated pathogens, killed antigens or their sub-units ${ }^{95}$. Vaccines based on whole pathogens are usually highly immunogenic but present safety concerns which often limit their use in high-risk populations (eg. pregnant women, elderly etc.). Recently developed vaccine (i.e. subunits, recombinant antigens, nucleotide, surface saccharides, etc.) are safer but typically generate weak immune responses. In light of this, such antigens generally require the use of adjuvants, which could improve both the delivery of the antigen and its immunostimulatory properties ${ }^{96}$. This concept of immune adjuvants was originally proposed by Gaston Ramon ${ }^{97}$, as "substances used in combination with a specific antigen that produce a more robust immune response than the antigen alone." The most common adjuvants are aluminum-based mineral salts ("alum") which have been FDA-approved for several decades ${ }^{98}$. Over the last decades, a few lipid-based adjuvants have been approved by regulatory agencies for seasonal and pandemic flu vaccines. Highly-functional polymeric adjuvants are also being investigated and this is an area of application where PPZs have shown particular promise. 
Already in 1998, a polyanionic PPZ derivative, poly[di(carboxylatophenoxy)-phosphazene] (PCPP), was identified as a promising adjuvant for commercial influenza vaccines since it could significantly improve the immunogenic responses in mice around ten-fold in comparison with the antigen alone ${ }^{99}$. It was found that the molecular weight of PCPP was also an important factor for boosting the immunogenic response. The longer PPZ (Mw $1500 \mathrm{kDa}$ ), when used as adjuvant for triple influenza vaccine, had 5 times higher hemagglutination inhibition antibodies than the same formulation prepared with the shorter PPZ (Mw $58 \mathrm{kDa})$.

The mechanism behind the immunostimulatory properties of PCPP is still unclear, but several explanations have been suggested ${ }^{100}$. First, PCPP does not seem to form a "depot" like alum ${ }^{99}$, but rather moves out of the injection site as dispersed hydrophilic complexes carrying the antigen. Second, PCPP forms non-covalent complexes with antigens, and the resulting nanoparticle structure seems to promote protein stability and more efficient antigen presentation to immune cells. This molecular complex formation process is likely affected by the molecular weight of PCPP, since larger polymers result in larger nanocomplexes and those induce higher amount of serum IgG titers in mice ${ }^{99}$. In addition, the carboxylic acid grafted groups on the PPZ backbone have inherent immunostimulatory activity, which could be mediated by TLRs and Mannose Receptors ${ }^{101,102}$.

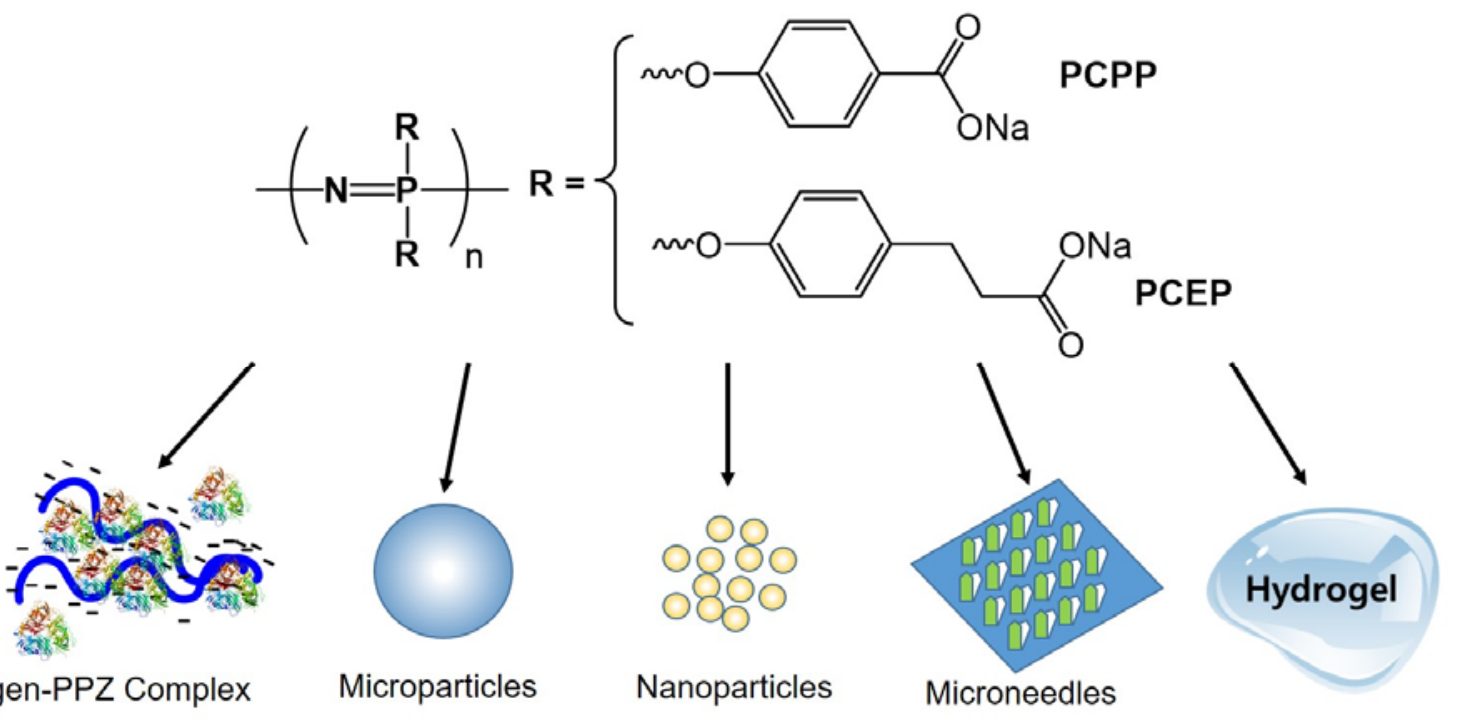


Figure 5. The chemical structure of PCPP and PCEP, two PPZ-based adjuvant materials, and the different types of vaccine formulations derived from them.

The use of PPZs as adjuvants is not only restricted to PCPP. Another PPZ with similar structure, poly[di(sodium carboxylatoethylphenoxy)phosphazene] (PCEP), can also boost immune responses in vaccination ${ }^{103,104}$. PCEP has been tested as adjuvant with different antigens: BSA, X-31 influenza, and hepatitis B surface antigen (HBsAg). When compared to PCPP, it was observed that PCEP trigger higher total IgG titers in vivo after several weeks. More specifically, PCEP used as adjuvant with relative low doses of X-31 stimulated high IgG titers and induced higher interferon- $\gamma$ production than alum or PCPP ${ }^{104}$. Either alum or PCPP mixing with X31 has a relatively high $\operatorname{IgG} 1 / \operatorname{IgG} 2$ a ratio of 1.3 , indicating a predominantly Th2 type immune response in mice. In contrast, PCEP-adjuvant X31 has a, $\operatorname{IgG1} / \mathrm{IgG} 2$ a ratio of 0.9 , presenting a balanced Th1/Th2 response with more Th1 isotype IgG2a. The reason behind this effect seems to be related to the different immunomodulatory effects of these adjuvants. While PCEP can contribute to stimulate both Type $1 \mathrm{~T}$ helper (Th1) and Type $2 \mathrm{~T}$ helper (Th2) responses, alum is mostly associated to Th2 responses. In fact, one of the main limitations of alum is its poor capacity to elicit Th1 responses, which is a main immune defense against intracellular pathogens.

Besides vaccine delivery by direct complexation with antigens, other PZZ systems have also been prepared, including nanocarriers (particles, liposomes) ${ }^{105}$, microparticles ${ }^{106-110}$, hydrogels ${ }^{111}$, and microneedle arrays ${ }^{112}$ (Figure 5). A particularly promising approach is the use of viscous PCPP-coated microneedle arrays that can penetrate the skin and degrade once in the body, releasing the antigen and the adjuvant. Andrianov and co-workers developed a single dose vaccine of HBsAg based on PCPP-coated microneedles to be used via transdermal delivery. In studies performed in pigs, this delivery system produced around 10 times higher IgG titers than the same formulation administered by intramuscular injection ${ }^{112}$. In summary, this study showed synergistic effects between the physical delivery exerted by the small needles and PPZs and demonstrated the potential of these material for minimally-invasive transdermal vaccination strategies. 
The first clinical trial on a PPZ-based adjuvant used PCPP co-administered with influenza antigens of three stains (A/Johannesburg/33/94 (H3N2)) in young adults and elderly ${ }^{113}$. This phase I study confirmed no serious adverse effects for this formulation and achieved better immune responses in young subjects, although elderly subjects still benefited from higher seroconversion rate in the PCPP-adjuvant group than in the standard vaccine group.

In addition to influenza, PCPP could be combined with HIV antigens to form macromolecular complexes. In vivo, PCPP-HIV Gag complexes activate more efficiently human dendritic cells (DCs) from adults and newborns and generate further cytokine production than the same antigen formulated with alum ${ }^{114}$. PCPP complexed with HIV vaccine formulations (ALVAC-HIV (vCP1521) primed with oligomeric gp160 (92TH023/LAI-DID) or Bivalent gp120 (CM235/SF2) boost) were evaluated for immune responses and safety in clinical studies. The results of these studies indicated the safety of the vaccines and their capacity to induce cell-mediated immunity 115,116. However, the protective effect of ALVAC-HIV (vCP1521) formulation was found to decay over time in these studies ${ }^{117}$. Another prime-boost Phase I study reported the combinations of ALVAC-HIV (vCP205) with oligomeric glycoprotein 160 (ogp160) and either PCPP or alum as adjuvants ${ }^{118}$. The ogp 160 subjects receiving the PCPP adjuvant had higher endpoint responses, including geometric mean antibody titers and T-Cell lympho-proliferation, than the subjects receiving the alum adjuvant (ClinicalTrials.gov Identifier NCT00004579).

In summary, for the moment the clinical evaluation has indicated that PPZ adjuvants are safe and immunostimulatory in humans, and that they can be translated to a variety of platforms including complexes, microparticles, gels, microneedles, etc. Further clinical studies are required to validate their utility for both influenza and HIV vaccination, particularly in comparison with other better-known adjuvant systems. 


\section{Conclusion}

Compared to other polymer families, stable PPZs have been synthesized relatively recently, and because of this and their anomalous inorganic backbone, they have not been explored as much as other biomaterials. Still, their many advantages have spurred increasing interest particularly concentrated in some areas like tissue engineering, gene delivery, protein delivery and vaccination. Particularly critical to this interest, is the biodegradability of most poly(organophosphazenes) and the chemical flexibility of these materials. Although other types of polymers can also be chemically diverse, engineers find appealing the special synthesis pathway of PPZs where a precursor is modified to tailor the material's structure and meet the requirements of specific applications. Such synthesis route simplifies adapting existing technologies to new areas of interest.

A pending challenge for the use of PPZs in pharmaceutics and medical devices is the optimization of this synthetic route to make it more cost-effective and eco-friendlier. Indeed, current synthetic pathways either rely on costly monomers and/or the use of high temperatures, organic solvents, and organic reactions proceeding under strict conditions. Cost considerations might be acceptable if the PPZ is integrated in large added-value systems where low amounts of material are needed (e.g. gene delivery systems) but can block further development in other applications.

Regulatory considerations have also been a concern for the development of PPZ-based systems since these materials do not have a long history of medical record comparable to other polymers. In this regard, good biocompatibility data observed in several animal models suggests that many PPZs can be considered for clinical translation. This good biocompatibility profile has been confirmed by the first clinical trials conducted with poly[di(carboxylatophenoxy)-phosphazene]. It is expected that all this new data on safety and bioactivity will encourage new laboratories to consider PPZ as important materials for the future design of advanced drug delivery systems.

\section{Acknowledgments}


This work has been funded by Ministerio de Economía y Competitividad (MINECO-RETOS, Grant MAT2017-84361-R, Feder Funds) and Xunta de Galicia (Grupos de Referencia Competitiva, Feder Funds) to MGF and Engineering and Physical Sciences Research Council Grants EP/N006615/1, EP/ N03371X/1 to CA. WHH was a recipient of a contract from EU Erasmus Mundus NanoFar joint doctorate program. 


\section{References}

1. Allcock, H. R., Chemistry and applications of polyphosphazenes; Wiley-Interscience, 2003.

2. Andrianov, A. K.; Allcock, H. R., Polyphosphazenes in Biomedicine, Engineering, and Pioneering Synthesis; ACS Publications, 2018.

3. Allcock, H., Phosphorus-nitrogen compounds: cyclic, linear, and high polymeric systems; Elsevier, 2012.

4. Mark, J. E.; Allcock, H. R.; West, R.; Oxford University Press, 2005, Chapter 3, p 62.

5. Dewar, M.; Lucken, E.; Whitehead, M., Journal of the Chemical Society (Resumed), 24231960.

6. Cowley, A.; Dewar, M.; Jackson, W. R., Journal of the American Chemical Society 90, 4185 1968.

7. Allcock, H. R.; Morozowich, N. L., Polymer Chemistry 3, 5782012.

8. Carampin, P.; Conconi, M. T.; Lora, S.; Menti, A. M.; Baiguera, S.; Bellini, S.; Grandi, C.; Parnigotto, P. P., Journal of Biomedical Materials Research Part A 80, 6612007.

9. Khalid, Z.; Ali, S.; Akram, M., International Journal of Polymeric Materials and Polymeric Biomaterials 67, 6932018.

10. Akram, M.; Wang, L.; Yu, H.; Amer, W. A.; Khalid, H.; Abbasi, N. M.; Chen, Y.; Saleem, M.; Tong, R., Progress in Polymer Science 39, 19872014.

11. Zheng, C.; Qiu, L.; Zhu, K., Polymer 50, 11732009.

12. Meng, L. J.; Xu, C. Q.; Liu, T. H.; Li, H.; Lu, Q. H.; Long, J. G., Polymer Chemistry 6, 3155 2015.

13. Hu, L.; Zhang, A. Q.; Liu, K.; Lei, S.; Ou, G. X.; Cheng, X. J., Rsc Advances 4, 357692014.

14. Qian, Y. C.; Huang, X. J.; Chen, C.; Ren, N.; Huang, X.; Xu, Z. K., Journal of Polymer Science Part A: Polymer Chemistry 50, 51702012.

15. Allcock, H. R., Science 193, 12141976.

16. De Jaeger, R.; Gleria, M., Progress in polymer science 23, 1791998.

17. Allcock, H. R. In Polyphosphazenes in Biomedicine, Engineering, and Pioneering Synthesis; American Chemical Society, 2018; Vol. 1298, Chapter 1, p 3.

18. Allcock, H. R.; Gardner, J.; Smeltz, K. M., Macromolecules 8, 361975.

19. Allcock, H. R.; Crane, C. A.; Morrissey, C. T.; Nelson, J. M.; Reeves, S. D.; Honeyman, C. H.; Manners, I., Macromolecules 29, 77401996.

20. Allcock, H. R.; Reeves, S. D.; Nelson, J. M.; Crane, C. A.; Manners, I., Macromolecules 30, 2213 1997.

21. Blackstone, V.; Pfirrmann, S.; Helten, H.; Staubitz, A.; Presa Soto, A.; Whittell, G. R.; Manners, I., Journal of the American Chemical Society 134, 152932012.

22. Honeyman, C. H.; Manners, I.; Morrissey, C. T.; Allcock, H. R., Journal of the American Chemical Society 117, 70351995.

23. Sennett, M. S.; Hagnauer, G. L.; Singler, R. E.; Davies, G., Macromolecules 19, 9591986.

24. Sohn, Y. S.; Cho, Y. H.; Baek, H.; Jung, O.-S., Macromolecules 28, 75661995.

25. Andrianov, A. K.; Chen, J.; LeGolvan, M. P., Macromolecules 37, 4142004.

26. Potta, T.; Chun, C.; Song, S.-C., Biomaterials 31, 81072010.

27. Lee, B. H.; Song, S.-C., Macromolecules 37, 45332004.

28. Qiu, L. Y.; Yan, M. Q., Acta biomaterialia 5, 21322009.

29. Weikel, A. L.; Cho, S. Y.; Morozowich, N. L.; Nair, L. S.; Laurencin, C. T.; Allcock, H. R., Polymer Chemistry 1, 14592010.

30. Cho, S. Y.; Allcock, H. R., Macromolecules 42, 44842009.

31. Huang, X.; Yin, L.; Tang, X., Science in China Series B: Chemistry 51, 462008.

32. Potta, T.; Chun, C.; Song, S.-C., Biomacromolecules 11, 17412010.

33. Rothemund, S.; Aigner, T. B.; Iturmendi, A.; Rigau, M.; Husár, B.; Hildner, F.; Oberbauer, E.; Prambauer, M.; Olawale, G.; Forstner, R., Macromolecular bioscience 15, 3512015.

34. Qian, Y.-C.; Chen, P.-C.; He, G.-J.; Huang, X.-J.; Xu, Z.-K., Molecules 19, 98502014. 
35. Nykänen, V. P. S.; Nykänen, A.; Puska, M. A.; Silva, G. G.; Ruokolainen, J., Soft Matter 7, 4414 2011.

36. Ren, N.; Huang, X. J.; Huang, X.; Qian, Y. C.; Wang, C.; Xu, Z. K., Journal of Polymer Science Part A: Polymer Chemistry 50, 31492012.

37. Heyde, M.; Moens, M.; Van Vaeck, L.; Shakesheff, K. M.; Davies, M. C.; Schacht, E. H., Biomacromolecules 8, 14362007.

38. Huang, X.; Huang, X. J.; Yu, A. G.; Wang, C.; Dai, Z. W.; Xu, Z. K., Macromolecular Chemistry and Physics 212, 2722011.

39. Chen, C.; Xu, H.; Qian, Y.-C.; Huang, X.-J., RSC Advances 5, 159092015.

40. Hsu, W. H.; Sánchez-Gómez, P.; Gomez-Ibarlucea, E.; Ivanov, D. P.; Rahman, R.; Grabowska, A. M.; Csaba, N.; Alexander, C.; Garcia-Fuentes, M., Advanced Therapeutics 2, 18001262019.

41. Teasdale, I.; Brüggemann, O., Polymers 5, 1612013.

42. Chun, C.; Lim, H. J.; Hong, K.-Y.; Park, K.-H.; Song, S.-C., Biomaterials 30, 62952009.

43. Allcock, H. R.; Pucher, S. R.; Scopelianos, A. G., Macromolecules 27, 10711994.

44. Allcock, H. R.; Pucher, S. R.; Scopelianos, A. G., Biomaterials 15, 5631994.

45. Deng, M.; Kumbar, S. G.; Wan, Y.; Toti, U. S.; Allcock, H. R.; Laurencin, C. T., Soft Matter 6, 31192010 .

46. Qian, Y.; Huang, X.; Xu, Z., Macromolecular Chemistry and Physics 216, 6712015.

47. DeCollibus, D. P.; Marin, A.; Andrianov, A. K., Biomacromolecules 11, 20332010.

48. Zheng, C.; Qiu, L.; Yao, X.; Zhu, K., International Journal of Pharmaceutics 373, 1332009.

49. Krogman, N. R.; Hindenlang, M. D.; Nair, L. S.; Laurencin, C. T.; Allcock, H. R., Macromolecules 41, 84672008.

50. Morozowich, N. L.; Weikel, A. L.; Nichol, J. L.; Chen, C.; Nair, L. S.; Laurencin, C. T.; Allcock, H. R., Macromolecules 44, 13552011.

51. Luten, J.; Van Steenis, J.; Van Someren, R.; Kemmink, J.; Schuurmans-Nieuwenbroek, N.; Koning, G.; Crommelin, D.; Van Nostrum, C.; Hennink, W., Journal of Controlled Release 89, 4832003.

52. Laurencin, C.; Morris, C.; Pierres-Jacques, H.; Schwartz, E.; Keaton, A.; Zou, L., Polymers For Advanced Technologies, 1992, 369, 359.

53. Nair, L. S.; Lee, D. A.; Bender, J. D.; Barrett, E. W.; E. Greish, Y.; Brown, P. W.; Allcock, H. R.; Laurencin, C. T., Journal of Biomedical Materials Research Part A: An Official Journal of The Society for Biomaterials, The Japanese Society for Biomaterials, and The Australian Society for Biomaterials and the Korean Society for Biomaterials 76, 2062006.

54. Sethuraman, S.; Nair, L. S.; El-Amin, S.; Nguyen, M.-T.; Singh, A.; Krogman, N.; Greish, Y. E.; Allcock, H. R.; Brown, P. W.; Laurencin, C. T., Acta biomaterialia 6, 19312010.

55. Andrianov, A. K.; Marin, A.; Martinez, A. P.; Weidman, J. L.; Fuerst, T. R., Biomacromolecules $19,34672018$.

56. Akram, M.; Wang, L.; Yu, H.; Khalid, H.; Abbasi, N. M.; ul-Abdin, Z.-.; Chen, Y.; Sun, R.; Jie, S.; Saleem, M., International Journal of Polymeric Materials and Polymeric Biomaterials 65, 581 2016.

57. Peng, Y.; Zhu, X.; Qiu, L., Biomaterials 106, 12016.

58. Bates, M. C.; Yousaf, A.; Sun, L.; Barakat, M.; Kueller, A., Regenerative Engineering and Translational Medicine2019.

59. Lakshmi, S.; Katti, D. S.; Laurencin, C. T., Advanced drug delivery reviews 55, 4672003.

60. Wilfert, S.; Iturmendi, A.; Schoefberger, W.; Kryeziu, K.; Heffeter, P.; Berger, W.; Brüggemann, O.; Teasdale, I., Journal of Polymer Science Part A: Polymer Chemistry 52, 2872014.

61. Vieira, A. C.; Vieira, J. C.; Ferra, J. M.; Magalhães, F. D.; Guedes, R. M.; Marques, A. T., Journal of the Mechanical Behavior of Biomedical Materials 4, 4512011.

62. Park, E.-S.; Maniar, M.; Shah, J., Journal of Controlled Release 40, 1111996.

63. Singh, A.; Krogman, N. R.; Sethuraman, S.; Nair, L. S.; Sturgeon, J. L.; Brown, P. W.; Laurencin, C. T.; Allcock, H. R., Biomacromolecules 7, 9142006. 
64. Andrianov, A. K., Polyphosphazenes for biomedical applications; John Wiley \& Sons, 2009.

65. Baillargeon, A. L.; Mequanint, K., Biomed Res Int 2014, 7613732014.

66. Lakshmi, S.; Katti, D.; Laurencin, C., Advanced drug delivery reviews 55, 4672003.

67. Wong, J. K.; Mohseni, R.; Hamidieh, A. A.; MacLaren, R. E.; Habib, N.; Seifalian, A. M., Trends in biotechnology, 2017.

68. Zhou, Z.; Liu, X.; Zhu, D.; Wang, Y.; Zhang, Z.; Zhou, X.; Qiu, N.; Chen, X.; Shen, Y., Advanced drug delivery reviews 115, 1152017.

69. Hanna, E.; Rémuzat, C.; Auquier, P.; Toumi, M., Journal of market access \& health policy 5, 12652932017.

70. Akinc, A.; Thomas, M.; Klibanov, A. M.; Langer, R., The journal of gene medicine 7, 6572005.

71. Chollet, P.; Favrot, M. C.; Hurbin, A.; Coll, J. L., The Journal of Gene Medicine: A crossdisciplinary journal for research on the science of gene transfer and its clinical applications 4,84 2002.

72. Hall, A.; Lächelt, U.; Bartek, J.; Wagner, E.; Moghimi, S. M., Molecular Therapy 25, 14762017.

73. Hall, A.; Larsen, A. K.; Parhamifar, L.; Meyle, K. D.; Wu, L.-P.; Moghimi, S. M., Biochimica et Biophysica Acta (BBA)-Bioenergetics 1827, 12132013.

74. De Wolf, H. K.; De Raad, M.; Snel, C.; Van Steenbergen, M. J.; Fens, M. H.; Storm, G.; Hennink, W. E., Pharmaceutical research 24, 15722007.

75. Amirshahi, N.; Alyautdin, R. N.; Sarkar, S.; Rezayat, S. M.; Orlova, M. A.; Trushkov, I. V.; Buchachenko, A. L.; Kuznetsov, D. A., Arch Med Res 39, 5492008.

76. Yang, Y.; Xu, Z.; Jiang, J.; Gao, Y.; Gu, W.; Chen, L.; Tang, X.; Li, Y., Journal of Controlled Release 127, 2732008.

77. Yang, Y.; Zhang, Z.; Chen, L.; Gu, W.; Li, Y., Biomacromolecules 11, 9272010.

78. Luten, J.; Van Steenbergen, M.; Lok, M.; De Graaff, A.; Van Nostrum, C.; Talsma, H.; Hennink, W., european journal of pharmaceutical sciences 33, 2412008.

79. Yang, Y.; Zhang, Z.; Chen, L.; Gu, W.; Li, Y., Bioconjug Chem 21, 4192010.

80. Zhang, P.; Zhang, Z.; Yang, Y.; Li, Y., International journal of pharmaceutics 392, 2412010.

81. Ma, C.; Zhang, X.; Du, C.; Zhao, B.; He, C.; Li, C.; Qiao, R., Bioconjugate chemistry 27, 1005 2016.

82. Yang, Y.; Xu, Z.; Chen, S.; Gao, Y.; Gu, W.; Chen, L.; Pei, Y.; Li, Y., International journal of pharmaceutics 353, 2772008.

83. Park, K.-H.; Song, S.-C., Journal of Biomaterials Science, Polymer Edition 16, 14212005.

84. Jiang, H.-L.; Kim, Y.-K.; Lee, S.-M.; Park, M.-R.; Kim, E.-M.; Jin, Y.-M.; Arote, R.; Jeong, H.J.; Song, S.-C.; Cho, M.-H., Archives of pharmacal research 33, 5512010.

85. Kim, Y.-M.; Song, S.-C., Biomaterials 35, 79702014.

86. Ryffel, B., Clinical immunology and immunopathology 83, 181997.

87. Lasek, W.; Zagożdżon, R.; Jakobisiak, M., Cancer Immunology, Immunotherapy 63, 4192014.

88. Gao, M.; Zhu, X.; Wu, L.; Qiu, L., Biomacromolecules 17, 21992016.

89. Martinez, A. P.; Qamar, B.; Fuerst, T. R.; Muro, S.; Andrianov, A. K., Biomacromolecules 18, 20002017.

90. Kang, G. D.; Song, S.-C., International journal of pharmaceutics 349, 1882008.

91. Kwak, H.; Shim, W.; Choi, M.; Son, M.; Kim, Y.; Yang, H.; Kim, T.; Lee, G.; Kim, B.; Kang, S., Journal of Controlled Release 137, 1602009.

92. Seong, J.-Y.; Jun, Y. J.; Kim, B. M.; Park, Y. M.; Sohn, Y. S., International journal of pharmaceutics 314, 902006.

93. Park, M.-R.; Chun, C.; Ahn, S.-W.; Ki, M.-H.; Cho, C.-S.; Song, S.-C., Journal of Controlled Release 147, 3592010.

94. Seo, B.-B.; Choi, H.; Koh, J.-T.; Song, S.-C., Journal of Controlled Release 209, 672015.

95. Singh, M.; O'Hagan, D. T., Pharmaceutical research 19, 7152002.

96. Eng, N. F.; Garlapati, S.; Gerdts, V.; Potter, A.; Babiuk, L. A.; Mutwiri, G. K., Current drug delivery 7, 132010 . 
97. Ramon, G., Ann. Inst. Pasteur 381924.

98. Exley, C.; Siesjö, P.; Eriksson, H., Trends in immunology 31, 1032010.

99. Payne, L. G.; Jenkins, S. A.; Woods, A. L.; Grund, E. M.; Geribo, W. E.; Loebelenz, J. R.; Andrianov, A. K.; Roberts, B. E., Vaccine 16, 921998.

100. Andrianov, A. K.; Marin, A.; Roberts, B. E., Biomacromolecules 6, 13752005.

101. Andrianov, A. K.; Sargent, J. R.; Sule, S. S.; Le Golvan, M. P.; Woods, A. L.; Jenkins, S. A.; Payne, L. G., Journal of bioactive and compatible polymers 13, 2431998.

102. Andrianov, A. K.; Marin, A.; Fuerst, T. R., Biomacromolecules 17, 37322016.

103. Andrianov, A. K.; Marin, A.; Chen, J., Biomacromolecules 7, 3942006.

104. Mutwiri, G.; Benjamin, P.; Soita, H.; Townsend, H.; Yost, R.; Roberts, B.; Andrianov, A. K.; Babiuk, L. A., Vaccine 25, 12042007.

105. Schulze, K.; Ebensen, T.; Babiuk, L. A.; Gerdts, V.; Guzman, C. A., Nanomedicine: Nanotechnology, Biology and Medicine 13, 21692017.

106. Payne, L. G.; Woods, A. L.; Jenkins, S. A.; US Patent 6207171: March 27, 2001.

107. Garlapati, S.; Eng, N. F.; Wilson, H. L.; Buchanan, R.; Mutwiri, G. K.; Babiuk, L. A.; Gerdts, V., Vaccine 28, 83062010.

108. Payne, L. G.; Jenkins, S. A.; Andrianov, A.; Roberts, B. E. In: Vaccine design; Springer, 1995, p 473.

109. Andrianov, A. K.; Jenkins, S. A.; Payne, L. G.; Roberts, B. E.; US Patent 5529777: Jun. 25, 1996.

110. Andrianov, A. K.; Chen, J., Journal of Applied Polymer science 101, 4142006.

111. Andrianov, A. K.; Chen, J.; Payne, L. G., Biomaterials 19, 1091998.

112. Andrianov, A. K.; DeCollibus, D. P.; Gillis, H. A.; Henry, H. K.; Marin, A.; Prausnitz, M. R.; Babiuk, L. A.; Townsend, H.; Mutwiri, G., Proceedings of the National Academy of Sciences 106, 189362009.

113. Le Cam, N. B.; Ronco, J.; Francon, A.; Blondeau, C.; Fanget, B., Research in Immunology 149, 191998.

114. Palmer, C. D.; Ninković, J.; Prokopowicz, Z. M.; Mancuso, C. J.; Marin, A.; Andrianov, A. K.; Dowling, D. J.; Levy, O., Biomaterials 35, 88762014.

115. Thongcharoen, P.; Suriyanon, V.; Paris, R. M.; Khamboonruang, C.; De Souza, M. S.; RattoKim, S.; Karnasuta, C.; Polonis, V. R.; Baglyos, L.; El Habib, R., JAIDS Journal of Acquired Immune Deficiency Syndromes 46, 482007.

116. Kim, J.; Kirsch, E.; Gilliam, B.; Michael, N.; VanCott, T.; Ratto-Kim, S.; Cox, J.; Nielsen, R.; Robb, M.; Caudrelier, P. 37th Annual Meeting of the Infectious Diseases Society of America, 1999.

117. Rerks-Ngarm, S.; Pitisuttithum, P.; Nitayaphan, S.; Kaewkungwal, J.; Chiu, J.; Paris, R.; Premsri, N.; Namwat, C.; de Souza, M.; Adams, E.; Benenson, M.; Gurunathan, S.; Tartaglia, J.; McNeil, J. G.; Francis, D. P.; Stablein, D.; Birx, D. L.; Chunsuttiwat, S.; Khamboonruang, C.; Thongcharoen, P.; Robb, M. L.; Michael, N. L.; Kunasol, P.; Kim, J. H., New England Journal of Medicine 361, 22092009.

118. O'connell, R. J.; Excler, J.-L.; Polonis, V. R.; Ratto-Kim, S.; Cox, J.; Jagodzinski, L. L.; Liu, M.; Wieczorek, L.; McNeil, J. G.; El-Habib, R., The Journal of infectious diseases 213, 19462016. 